\title{
Interference Characterization and Spectrum Sharing in Large Wireless Networks
}

\author{
by \\ Muhammad Al-Juaid, B. Sc., M. Sc. \\ A thesis submitted to the Faculty of Graduate Studies and Research \\ in partial fulfillment of the requirements for the degree of \\ Doctor of Philosophy in Electrical and Computer Engineering
}

Ottawa-Carleton Institute for Electrical and Computer Engineering (OCIECE)

Department of Systems and Computer Engineering

Carleton University

Ottawa, Ontario, Canada, K1S 5B6

July 2010

(C) Copyright 2010, Muhammad Al-Juaid 


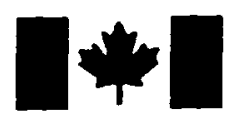

\author{
Library and Archives \\ Canada \\ Published Heritage \\ Branch \\ 395 Wellington Street \\ Ottawa ON K1A ON4 \\ Canada
}

Bibliotheqque et Archives Canada

Direction du

Patrimoine de l'édition

395, rue Wellington Ottawa ON K1A ON4

Canada
Your file Votre reférence

ISBN: 978-0-494-70541-4

Our file Notre référence

ISBN: 978-0-494-70541-4
NOTICE:

The author has granted a nonexclusive license allowing Library and Archives Canada to reproduce, publish, archive, preserve, conserve, communicate to the public by telecommunication or on the Internet, loan, distribute and sell theses worldwide, for commercial or noncommercial purposes, in microform, paper, electronic and/or any other formats.

The author retains copyright ownership and moral rights in this thesis. Neither the thesis nor substantial extracts from it may be printed or otherwise reproduced without the author's permission.
AVIS:

L'auteur a accordé une licence non exclusive permettant à la Bibliothèque et Archives Canada de reproduire, publier, archiver, sauvegarder, conserver, transmettre au public par télécommunication ou par l'Internet, prêter, distribuer et vendre des thèses partout dans le monde, à des fins commerciales ou autres, sur support microforme, papier, électronique et/ou autres formats.

L'auteur conserve la propriété du droit d'auteur et des droits moraux qui protège cette thèse. Ni la thèse ni des extraits substantiels de celle-ci ne doivent être imprimés ou autrement reproduits sans son autorisation.
In compliance with the Canadian Privacy Act some supporting forms may have been removed from this thesis.

While these forms may be included in the document page count, their removal does not represent any loss of content from the thesis.
Conformément à la loi canadienne sur la protection de la vie privée, quelques formulaires secondaires ont été enlevés de cette thèse.

Bien que ces formulaires aient inclus dans la pagination, il n'y aura aucun contenu manquant.

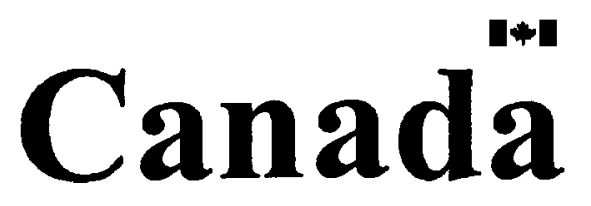




\section{Abstract}

Empowered with advancements in wireless technologies, radio spectrum regulators are formulating a new spectrum management paradigm. Under this paradigm, it is possible for a secondary user (SU) who does not necessarily have a spectrum license to share a spectrum band with a primary user (PU) (the license holder) provided that operations of the PU are not disturbed. A more interesting scenario is when there are many SUs willing to utilize the spectrum band of the PU. The aggregate interference power received by the PU due to the transmissions of SUs is a key parameter determining the availability of spectrum sharing opportunities. One of our contributions is a cumulant-based characterization of this aggregate interference power. We introduce a comprehensive method to determine the cumulants under various system and channel conditions. These cumulants are utilized to understand the dynamics of the aggregate interference power, to approximate its distribution, and hence to investigate the spectrum sharing opportunities.

Another contribution is the investigation of the Gaussianity of the aggregate interference. We cast in a single mathematical framework the observations scattered across the literature about the Gaussianity of the distribution of the aggregate interference power. Moreover, we discuss the effect of different system and channel parameters on the convergence of the distribution of the aggregate interference to a Gaussian distribution.

Furthermore, this thesis studies the effect of the spatial size of the secondary 
network on spectrum sharing opportunities. We verify that asymptotic results obtained for infinite fields are applicable for finite but relatively large fields as well. We also demonstrate that in some cases, however, asymptotic results are too pessimistic hiding some spectrum sharing opportunities.

Identifying the dominant region of the secondary network that would impact spectrum sharing opportunities is among our contributions. Results reveal that far interferers may tangibly contribute to spectrum sharing decisions when a higher approximation accuracy is required or when a wide exclusion region (within which no SUs are allowed to transmit) is considered. However, the dominant region shrinks with an increase in the path-loss exponent or in the level of the interference threshold specified by the PU or a regulator. 


\section{Acknowledgements}

I am so grateful to God (Allah) for his uncountable bounties on me including ability to perform this research and to write this thesis. I would like also to acknowledge my thesis supervisor, Dr. Halim Yanikomeroglu, for his patience, guidance, encouragement, support and help during the course of this research. Moreover, many thanks and appreciation should go to Saudi Aramco, Saudi Arabia, for awarding me the scholarship to pursue my $\mathrm{PhD}$ studies. Finally, I would like to express my deep gratitude to all my family members and friends for their support and prayers. 


\section{Contents}

$\begin{array}{ll}\text { Abstract } & \text { ii }\end{array}$

Acknowledgements

Table of Contents $\quad$ iv

List of Figures $\quad x$

List of Tables $\quad$ xiv

Nomenclature $\quad$ Xv

1 Introduction 1

1.1 Motivations .......................... 1

1.1.1 Cumulant-based Characterization of the Aggregate Interference

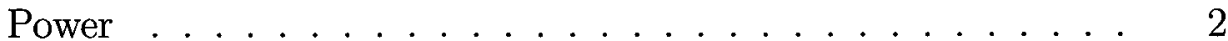

1.1.2 Gaussianity of the Distribution of the Aggregate Interference Power ............................ 3

1.1.3 Impact of the Spatial Size of the Secondary Network on Spectrum Sharing ..................... 3

1.1.4 Dominant Regions in Large Secondary Networks . . . . . . . . 4

1.2 Overview .......................... 4

1.3 Contributions ......................... 4 
1.4 Published, Submitted, and Proposed Manuscripts . . . . . . . . 6

1.5 Organization $\ldots \ldots \ldots \ldots$

2 Background and Literature Review 9

2.1 Spectrum Sharing . . . . . . . . . . . . . . . . . 9 9

2.1.1 Spectrum Sharing Schemes . . . . . . . . . . . . . . 12

2.1 .2 Metrics for Harmful Interference . . . . . . . . . . . . . . 12

2.2 Related work ..................... 13

2.2.1 Spectrum Sharing in Large Wireless Networks . . . . . . . . 13

2.2.2 Characterization of Interference in Large Wireless Networks . 15

2.3 Statistical and Mathematical Preliminaries . . . . . . . . . . . . 17

2.3.1 Poisson Point Processes $[1-3] \ldots \ldots \ldots$. . . . . . . 17

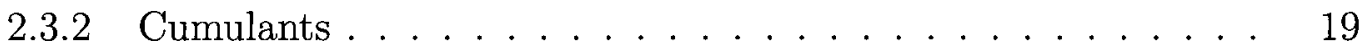

3 System Model $\quad 21$

3.1 Spatial Distribution of Secondary Users . . . . . . . . . . . 21

3.2 Aggregate Interference Power . . . . . . . . . . . . . . 22

3.3 Path-Loss Models . . . . . . . . . . . . . . . . . 24

3.4 Harmful Interference Metric . . . . . . . . . . . . . 25

3.5 Summary ............................ 26

4 Preliminary Results on Interference Characterization 27

4.1 Asymptotic Analysis of the Average Interference Power Generated by a Wireless Sensor Network Towards a Primary User . . . . . . . . 27

4.1.1 Spectrum Sharing for Wireless Sensor Networks . . . . . . . . 28

4.1.2 Average Interference Power . . . . . . . . . . . . . 29

4.1.3 Shape I: Sensor Field of Annular Sector Shape . . . . . . . . . 31

4.1.4 Shape II: Sensor Field of Rectangular Shape . . . . . . . . . 37 
4.1.5 Upper Bounds on the Interference of a Sensor Field . . . . . 43

4.1.5.1 Average Interference Power of any Sensor Field with an Arbitrary Shape . . . . . . . . . . . . 43

4.1.5.2 An Upper Bound on Interference Probability . . . 44

4.2 Upper Bound on the Interference Probability . . . . . . . . . . 45

4.2 .1 Objective and Approach $\ldots \ldots \ldots \ldots \ldots$

4.2.2 Mean of the Aggregate Interference . . . . . . . . . . 46

4.2 .2 .1 The Formulation . . . . . . . . . . . . 47

4.2.2.2 Effect of Field Size and Location on $\mu_{A} \ldots \ldots \ldots$

4.2.3 Variance of the Aggregate Interference $\ldots \ldots \ldots \ldots$

4.2.3.1 The Formulation . . . . . . . . . . . . . 50

4.2.3.2 Effect of Field Size and Location on $\sigma_{A}^{2} \ldots \ldots \ldots$

4.2.4 Upper Bound on the Interference Probability . . . . . . . . . 52

4.2.4.1 The Formulation . . . . . . . . . . . . . 52

4.2.4.2 Effect of Field Size and Location on the Interference Probability ................... 53

4.2.5 Effect of Field Size and Location on Spectrum Sharing Oppor-

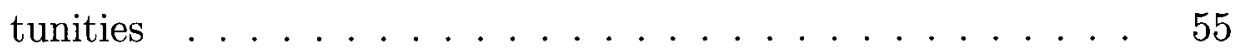

4.2.5.1 Field Size and Spectrum Sharing Opportunities . . . 57

4.2.5.2 Field Location and Spectrum Sharing Opportunities 59

4.3 Conclusions . . . . . . . . . . . . . . . . . . . . 60

5 Cumulants-Based Characterization of the Aggregate Interference $\begin{array}{ll}\text { Power and Spectrum Sharing } & 62\end{array}$

5.1 Introduction . . . . . . . . . . . . . . 63

5.2 Cumulants of $I_{A} \ldots \ldots \ldots \ldots \ldots \ldots \ldots \ldots \ldots \ldots \ldots \ldots \ldots$

5.3 Effect of the Distribution of $X$ on Cumulants $\ldots \ldots \ldots 66$ 
5.3 .1 Multipath Fading . . . . . . . . . . . . . . . . 67

5.3 .2 Shadow Fading . . . . . . . . . . . . . . 67

5.3 .3 Composite Fading . . . . . . . . . . . . . . . 68

5.4 Generalizations of the Cumulant-Based Characterization . . . . . 68

5.4.1 A Field with an Irregular Shape . . . . . . . . . . . . . 68

5.4 .2 A Field of Heterogeneous Networks _ . . . . . . . . . 69

5.4 .3 Many Primary Users . . . . . . . . . . . . . . . . . 69

5.5 Cumulants-based Approximations of the Distribution of the Aggregate Interference Power $\ldots \ldots \ldots \ldots \ldots$. . . . . . . . 70

5.5.1 Edgeworth Series Expansion . . . . . . . . . . . 70

5.5 .2 Shifted Lognormal . . . . . . . . . . . . . 71

5.5.3 General Remarks on the Approximations . . . . . . . . . . 72

5.5.4 Approximation and Simulation Results for the Distribution of $I_{A} 73$

5.6 A Cumulant-Based Investigation of the Impact of Secondary Users'

Field Size on Spectrum Sharing Opportunities _. . . . . . . . . 73

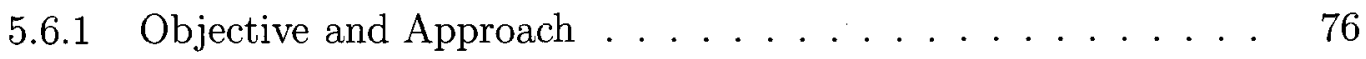

5.6.2 Effect of Spatial Size of the Field and SU-TXs Density on the Cumulants . . . . . . . . . . . . . . 77

5.6.3 Effect of Field Size on Spectrum Sharing Opportunities . . . 80 5.6.3.1 Spectrum Sharing Opportunities _... . . . . 80

5.6.3.2 Field Size and Spectrum Sharing Opportunities _.. . 81

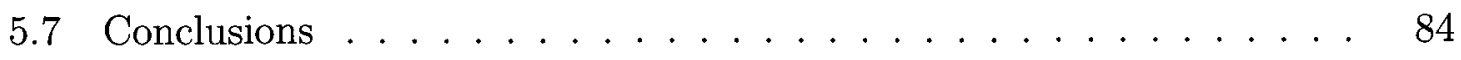

6 The Gaussian Convergence of the Distribution of the Aggregate In$\begin{array}{ll}\text { terference Power } & \mathbf{8 6}\end{array}$

6.1 Introduction . . . . . . . . . . . . . . 86

6.2 Berry-Esseen Bound for the Distribution of $I_{A} \ldots \ldots \ldots 8$ 
6.2.1 Exclusion Region $\left(r_{o} \geq r_{c}\right) \ldots \ldots \ldots \ldots$

6.2 .2 No Exclusion Region $\left(r_{o}=0\right) \ldots \ldots \ldots \ldots$

6.3 Effect of Fading Distributions on the Gaussian Approximation of $I_{A}$. 95

6.3.1 Case I: Without Multipath Fading and without Shadow Fading 95

6.3.2 Case II: With Multipath Fading but without Shadow Fading . 96

6.3.3 Case III: With Shadow Fading but without Multipath Fading 96

6.3.4 Case IV: With Composite Fading . . . . . . . . . . . 97

6.3.5 General Remarks . . . . . . . . . . . . . . . 98

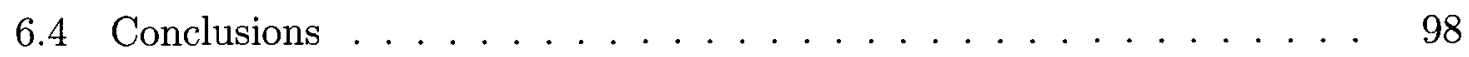

7 Identifying Boundaries of Dominant Regions Dictating Spectrum $\begin{array}{ll}\text { Sharing Opportunities } & 100\end{array}$

7.1 Introduction . . . . . . . . . . . . . . . . . . . . 101

7.2 Cumulants of $I_{A}$ and the Interference Probability $\ldots \ldots \ldots$

7.3 The Dominant Region . . . . . . . . . . . . . . . . . 103

7.3.1 A Cumulant-Based Approach . . . . . . . . . . . . 104

7.3.2 An Interference Probability-Based Approach . . . . . . . . 105

7.3.3 Implications of the Dominant Region . . . . . . . . . . . 109

7.4 Conclusions . . . . . . . . . . . . . . . . . 111

8 Conclusions and Suggested Future Work 112

8.1 Summary . . . . . . . . . . . . . . . . . . . . 112

8.2 Contributions . . . . . . . . . . . . . . . . . . 113

8.2.1 Cumulant-based Characterization of the Aggregate Interference Power . . . . . . . . . . . . . . . . 113

8.2.2 Gaussianity of the Distribution of the Aggregate Interference Power . . . . . . . . . . . . . . . . . 113 
8.2.3 Impact of the Spatial Size of the Secondary Network on Spectrum Sharing . . . . . . . . . . . . . . 114

8.2.4 Dominant Regions in Large Secondary Networks . . . . . . . 114

8.3 Suggested Future Work . . . . . . . . . . . . . . . . . . . 115

8.3.1 Spatially Clustered Secondary Users _. . . . . . . . . 115

8.3.2 Exact Analysis of Spectrum Sharing for a Secondary Network Overlapped with Spatially Distributed Many Primary Users . 115

8.3.3 Further Accurate Approximating Distributions for the Aggregate Interference Power . . . . . . . . . . . 116

8.3.4 Effect of Correlation among the $X_{i}^{\prime}$ ' $\ldots \ldots \ldots 116$

$\begin{array}{ll}\text { Appendices } & 130\end{array}$

$\begin{array}{ll}\text { A The Berry-Esseen Theorem } & 131\end{array}$

B Derivation of the Cumulant-Based Berry-Esseen Bound 132

C Generalization of the Berry-Esseen Bound for a $d$-dimensional Space 


\section{List of Figures}

3.1 Field layout. . . . . . . . . . . . . . . . . . . . 22

4.1 Representing a sensor field by a signal virtual node generating equivalent level of average interference power. . . . . . . . . . . . 31

4.2 A sensor field with the shape of an annular sector. . . . . . . . . . 32

4.3 Normalized $K$, i.e, $K / \lambda r_{o}^{2}$, vs. $L / r_{o}$ for different values of $n . \ldots 33$

4.4 Effective areas of Shape I when $L \gg r_{o}$ and $L \ll r_{o} \ldots \ldots \ldots$

4.5 A sensor field of a rectangular shape (Shape II). (a) General alignment;

(b) Worst-case alignment (given $y_{o}$ is fixed). . . . . . . 38

4.6 Normalized $K$, i.e, $K / \lambda r_{o}^{2}$, for Shape II for $n=3 \ldots \ldots \ldots 41$

4.7 Impact of the changes in $L$ on normalized $K$, i.e, $K / \lambda r_{o}^{2}$, for Shape II. (a) $W / r_{o}=100 ;$ (b) $W / r_{o}=0.01 \ldots \ldots \ldots \ldots \ldots \ldots \ldots \ldots$

4.8 Bounding a field of an arbitrary shape by one of the regular shapes considered in this chapter. . . . . . . . . . . . . 43

4.9 Effect of field size on the mean of the aggregate interference $\left(r_{o}=1000\right.$ $\mathrm{m}$; see Table 4.1 for other assumptions used to produce this plot). . . 48

4.10 Effect of $r_{o}$ on the mean of the aggregate interference $(L=1000 \mathrm{~m}$; see Table 4.1 for other assumptions used to produce this plot). . . . . . 49

4.11 Effect of field size on variance of the aggregate interference $\left(r_{o}=1000\right.$ $\mathrm{m}$; see Table 4.1 for other assumptions used to produce this plot). . . 
4.12 Effect of $r_{o}$ on the variance of the aggregate interference $(L=1000 \mathrm{~m}$; see Table 4.1 for other assumptions used to produce this plot). . . . 52

4.13 Effect of field size on interference probability $\left(r_{o}=1000 \mathrm{~m}\right.$; see Table 4.1 for other assumptions used to produce this plot). . . . . . . . 54

4.14 Effect of field location on interference probability $(L=1000 \mathrm{~m}$; see Table 4.1 for other assumptions used to produce this plot). . . . . . . .

4.15 Field size and spectrum sharing opportunities $\left(r_{o}=1000 \mathrm{~m}\right.$; see Table 4.1 for other assumptions used to produce this plot). . . . . . 57

4.16 Field location and spectrum sharing opportunities $(L \rightarrow \infty$; see Table 4.1 for other assumptions used to produce this plot). . . . . . . . .

5.1 The CCDF of $I_{A}\left(r_{o}=10 \mathrm{~m}, L=1000 \mathrm{~m}, n=3, \lambda=0.01\right.$ node $/ \mathrm{m}^{2}$, $\theta=2 \pi$, no multipath fading, no shadow fading). (a) The CCDF on a linear scale. (b) The upper tail of the CCDF (log-log scale). . . . . 74

5.2 The CCDF of $I_{A}\left(r_{o}=10 \mathrm{~m}, L=1000 \mathrm{~m}, n=3, \lambda=0.01\right.$ node $/ \mathrm{m}^{2}$, $\theta=2 \pi$, Rayleigh fading, $6 \mathrm{~dB}$ shadow fading). (a) The CCDF on a linear scale. (b) The upper tail of the CCDF (log-log scale). The curve for the Edgeworth approximation is excluded because it fails to generate a valid CCDF curve for heavily skewed distributions. We show a lognormal approximation instead. . . . . . . . . . . . . 75

5.3 The effect of $L$ on $A_{\text {eff }}$, and hence on $\kappa_{m}$, for the case of no exclusion region around the PU-RX $\left(r_{c}=1\right.$ meter, $\theta=2 \pi$, and $\left.n=3\right) \ldots$

5.4 The effect of $L$ on $A_{\text {eff }}$, and hence on $\kappa_{m}$, for the case of an exclusion region of $r_{o}=10$ meters $\left(r_{c}=1\right.$ meter, $\theta=2 \pi$, and $\left.n=3\right) \ldots \ldots$ 
5.5 Field size and spectrum sharing opportunities $\left(r_{o}=10 \mathrm{~m}, n=3, r_{c}=1\right.$ $\mathrm{m}, \theta=2 \pi, \lambda=0.01$ node $/ \mathrm{m}^{2}$, no multipath fading, no shadow fading). The dashed line corresponds to $P_{\text {int }}=\beta=0.1$, which divides the figure into two regions: a non-interfering region (the lower part of the figure), and an interfering region (the upper part of the figure). The values of $I_{t h}$ are properly chosen from the corresponding CCDF curve; see for example Fig. 5.1. . . . . . . . . . . . . . .

5.6 Field size and spectrum sharing opportunities under different values of $r_{o}\left(n=3, r_{c}=1 \mathrm{~m}, \theta=2 \pi, \lambda=0.01\right.$ node $/ \mathrm{m}^{2}$, no multipath fading, no shadowing, $I_{t h}=0.009$ ). The dashed line corresponds to $P_{\text {int }}=\beta=$ 0.1 , which divides the figure into two regions: a non-interfering region (the lower part of the figure), and an interfering region (the upper part of the figure). . . . . . . . . . . . . . . . .

6.1 Monte-Carlo simulation-based histograms for the normalized $I_{A}$, i.e., $\frac{I_{A}-\tilde{\mu}_{A}}{\sigma_{A}}$, for different values of $\lambda\left(r_{o}=10\right.$ meters, $r_{c}=1$ meter, $n=3$, no multipath fading, and no shadow fading). (a) $\lambda=0.001$ nodes $/$ meter $^{2}$. (b) $\lambda=0.01$ nodes $/$ meter $^{2}$. (c) $\lambda=0.1$ nodes $/$ meter $^{2} \ldots \ldots$. . . .

6.2 Monte-Carlo simulation-based CDF for the normalized $I_{A}$, i.e., $\frac{I_{A}-\tilde{\mu}_{A}}{\sigma_{A}}$, for different values of $\lambda\left(r_{o}=10\right.$ meters, $r_{c}=1$ meter and $\left.n=3\right)$.

6.3 Monte-Carlo simulation-based CDF for the normalized $I_{A}$, i.e., $\frac{I_{A}-\tilde{\mu}_{A}}{\sigma_{A}}$, for different values of $r_{o}\left(\lambda=0.01\right.$ nodes $/$ meter $^{2}, r_{c}=1$ meter and

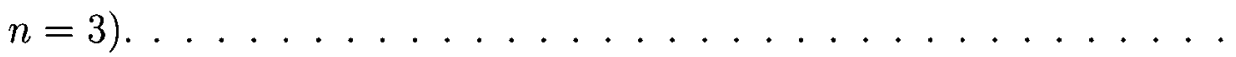

6.4 Values of $\tilde{\mu}_{3}(X) /\left[\tilde{\mu}_{2}(X)\right]^{\frac{3}{2}}$ for different values of $\sigma_{s} \ldots \ldots \ldots$. . . 97

6.5 Monte-Carlo simulation-based CDF for the normalized $I_{A}$, i.e., $\frac{I_{A}-\tilde{\mu}_{A}}{\sigma_{A}}$, for different fading scenarios $\left(r_{o}=10\right.$ meters, $r_{c}=1$ meter, $n=3$, and $\lambda=0.01$ nodes $/$ meter $\left.^{2}\right) . \ldots \ldots \ldots \ldots$ 
7.1 Layout of a partitioned secondary network. In this chapter, we consider $L \rightarrow \infty$. The dominant region $\mathcal{R}_{d}$ has an inner radius of $r_{o}$ and an outer radius $r_{o}+L_{d}$. The inner white circle is the exclusion region. The region $\mathcal{R}_{t}=\mathcal{R}_{d} \cup \mathcal{R}_{2}$ corresponds to the whole secondary network. 102

7.2 The CCDF of the aggregate interference power based on a shifted lognormal approximation for different values of $L\left(r_{o}=10 \mathrm{~m}, r_{c}=1 \mathrm{~m}\right.$, $\lambda=0.01$ nodes $/ \mathrm{m}^{2}, n=3$, Rayleigh fading, and $6 \mathrm{~dB}$ shadowing). $\quad 107$

7.3 The CCDF of the aggregate interference power based on a shifted lognormal approximation for different values of $L\left(r_{o}=1 \mathrm{~m}, r_{c}=1 \mathrm{~m}\right.$, $\lambda=0.01$ nodes $/ \mathrm{m}^{2}, n=3$, Rayleigh fading, and $6 \mathrm{~dB}$ shadowing). $\quad 108$

7.4 Relative approximation error in the interference probability for different values of $I_{t h}\left(r_{o}=10 \mathrm{~m}, r_{c}=1 \mathrm{~m}, \lambda=0.01\right.$ nodes $/ \mathrm{m}^{2}, n=3$, Rayleigh fading, and $6 \mathrm{~dB}$ shadowing). The values of $I_{t h}$ are chosen to correspond to the interference probability of 0.1 and 0.01 , respectively, that a PU-RX would experience from an infinite secondary network. 109

7.5 Relative approximation error in the interference probability for different values of $I_{t h}\left(r_{o}=1 \mathrm{~m}, r_{c}=1 \mathrm{~m}, \lambda=0.01\right.$ nodes $/ \mathrm{m}^{2}, n=3$, Rayleigh fading, and $6 \mathrm{~dB}$ shadowing). The values of $I_{t h}$ are chosen to correspond to the interference probability of 0.1 and 0.01 , respectively, that a PU-RX would experience from an infinite secondary network. 


\section{List of Tables}

4.1 System and Propagation Parameters Used to Produce Plots . . . . . 49

4.2 Summary of Asymptotic Expressions . . . . . . . . . . . . . 55

7.1 $L_{d}$ calculated for different values of maximum approximation errors in the first three cumulants. Calculations are repeated for different values of $r_{o}$ and $n$ to reflect their effects. . . . . . . . . . 106 


\section{Nomenclature}

\section{Acronyms}

\begin{tabular}{ll}
\hline Acronym & Meaning \\
\hline \hline PU & Primary User \\
SU & Secondary User \\
TX & Transmitter \\
RX & Receiver \\
CR & Cognitive Radio \\
WSN & Wireless Sensor Network \\
CDF & Cumulative Distribution Function \\
CCDF & Complimentary Cumulative Distribution Function \\
CLT & Central Limit Theorem \\
i.i.d. & independent and identically distributed \\
PDF & Probability Density Function \\
PP & Point Process \\
BPP & Binomial Point Process \\
PPP & Poisson Point Process \\
RV & Random Variable \\
\hline
\end{tabular}




\section{Mathematical Operators}

\begin{tabular}{ll}
\hline Operator & Meaning \\
\hline \hline $\mathbb{E}[X]$ & Expected value of RV $X$ \\
$\mathbb{P}(A)$ & Probability of event A \\
$\Gamma()$. & Gamma Function \\
\hline
\end{tabular}


Frequently Used Symbols

\begin{tabular}{|c|c|}
\hline Symbol & Meaning \\
\hline$r_{o}$ & Radius of the exclusion region \\
\hline$L$ & Spatially radial depth of the field/netwrok \\
\hline$n$ & Path-loss exponent \\
\hline$I_{A}$ & Aggregate interference power \\
\hline$I_{i}$ & $\begin{array}{l}\text { Individual Interference Power due to the Transmission of } \\
\text { Node/Interferer } i\end{array}$ \\
\hline$I_{t h}$ & Interference threshold specified by a regulator or $\mathrm{PU}$ \\
\hline$r_{i}$ & The distance between a $\mathrm{SU} i$ and the $\mathrm{PU}$ \\
\hline$g\left(r_{i}\right)$ & Distance-dependent attenuation model (or path-loss model) \\
\hline$r_{c}$ & The radius at which the slope of the path-loss model starts changing \\
\hline$\kappa_{m}\left(I_{A}\right)$ & the $m$ th cumulant of the aggregate interference power \\
\hline$\tilde{\mu}_{m}(X)$ & $m$ th raw moment of $X$, i.e., $\mathbb{E}\left[X^{m}\right]$ \\
\hline$m$ & The order of the cumulant or moment \\
\hline$X_{i}$ & $\begin{array}{l}\text { Positive RV modeling different system and channel parameters } \\
\text { (except the distance-dependent attenuation) affecting the received } \\
\text { power at the PU from the transmission of } \mathrm{SU} i\end{array}$ \\
\hline$\lambda$ & Intensity (density) of a PPP (modeling density of active SU-TXs) \\
\hline$\theta$ & $\begin{array}{l}\text { The angle over which the network (field) of SUs spans as seen by a } \\
\text { PU }\end{array}$ \\
\hline $\mathbb{R}^{d}$ & d-dimensional Euclidean space \\
\hline$d$ & Number of the dimensions of the space \\
\hline$\beta$ & Maximum interference probability as specified by a regulator or $\mathrm{PU}$ \\
\hline$\mu_{A}$ & Mean of the aggregate interference power $\left(I_{A}\right)$ \\
\hline$\mu_{I}$ & Mean of the individual interference power $\left(I_{i}\right)$ \\
\hline$\mu_{X}$ & Mean of the random variable $X$ \\
\hline$\sigma_{A}^{2}$ & Variance of the aggregate interference power $\left(I_{A}\right)$ \\
\hline$\sigma_{X}^{2}$ & Variance of the random variable $X$ \\
\hline
\end{tabular}




\section{Chapter 1}

\section{Introduction}

The management of the radio spectrum is going through a paradigm shift, allowing secondary users (SUs) ${ }^{1}$ to share spectrum bands with Primary Users (PUs) who hold the licenses of these bands. This spectrum sharing would be permissible under the constraint that activities of SUs in these bands do not harmfully disturb the operation of the PUs [4-6]. An important parameter in determining a spectrum sharing opportunity is the level of interference power that secondary users may generate towards primary users. This thesis deals with different aspects related to interference characterization and spectrum sharing in large secondary networks. The rest of this chapter highlights motivations, contributions, and organization of this thesis. This chapter also provides a list of publications resulted from the work presented in this thesis.

\subsection{Motivations}

An initial motivation for the work presented in this thesis is the lack of clear understanding of the impact of the spatial size of a wireless network on the aggregate interference, and on spectrum sharing opportunities. To the best of our knowledge,

\footnotetext{
${ }^{1}$ In this thesis, the "secondary user" means a radio device or system that can opportunistically access in a non-interfering basis an under-utilized spectrum band licensed to other users. The term "secondary user" might be used differently by frequency regulators.
} 
there is no work in the literature devoted to study this impact. Such study and understanding would provide answers to some important and interesting questions such as the following:

- How do changes in the spatial size of the network affect the mean, variance and distribution of the aggregate interference?

- Does an increase in the spatial size of the secondary network eliminate spectrum sharing opportunities?

- Could asymptotic results (obtained for a network with an infinite spatial size) be applied for a network with a finite size? Would these results be too conservative hiding spectrum sharing opportunities?

- What is the smallest portion (dominant region) of the secondary network that dictates spectrum sharing opportunities? How does the boundary of this region change with changes in system parameters?

Further motivations of the work reported in this thesis are discussed in the following subsections.

\subsubsection{Cumulant-based Characterization of the Aggregate Interference Power}

The importance of characterizing the aggregate interference power generated by a wireless network has increased with the emergence of spectrum sharing and cognitive radio. A cumulant-based characterization of this aggregate interference is an attractive approach. A number or recent papers in the literature have dealt with cumulants of the aggregate interference but under specific scenarios. For example, [7] and [8] only consider the first cumulant, i.e., the mean. The authors in [9] and [10] deal with cumulants for non-fading scenarios, [11] provides an integral form to compute the cu-

mulants for out-of-cell interference in CDMA networks, and [12] considers an infinite 
field with a very small exclusion region. Extending these results and generalizing them for a wide range of scenarios are of great importance and advantage to study the spectrum sharing in large secondary networks.

\subsubsection{Gaussianity of the Distribution of the Aggregate Interference Power}

The aggregate interference can be considered as the sum of a large number of independent interference signals. As the number of interfering nodes increases, there might be a tendency to approximate the distribution of the aggregate interference power by a Gaussian random variable given that the individual interference signals are independent. However, some scattered observations in literature suggest that this Gaussian approximation is not valid, except under some specific scenarios $[11,13-16]$. Therefore, there is a need to have a thorough investigation of the Gaussian convergence of the distribution of the aggregate interference power, and to cast these scattered observations in a single mathematical framework.

\subsubsection{Impact of the Spatial Size of the Secondary Network on Spectrum Sharing}

Previous works such as $[9,12,17,18]$ studied the effect of different system parameters on spectrum sharing opportunities. However, a parameter that has received little attention is the spatial size of the secondary network. Usually, the spatial size is assumed to be infinite. Using results developed for infinite networks might be too pessimistic leading to missing spectrum sharing opportunities. This concern about the applicability of the results of infinite networks could be addressed properly by studying the behavior of the aggregate interference, and hence the spectrum sharing opportunities, with respect to the changes in the spatial size of the secondary network. To the best of our knowledge, current literature does not have such studies. 


\subsubsection{Dominant Regions in Large Secondary Networks}

While there are some comments in literature (e.g., in [19] and [20]) indicating that the aggregate interference is dominated by the nearby interferers to the victim receiver, there is to the best of our knowledge no work devoted to precisely identifying the boundary of the dominant region. Thus, a contribution is required to fill this gap, especially in the context of spectrum sharing.

\subsection{Overview}

This thesis can be considered as a study of the effect of different system and channel parameters (in particular the spatial size of the network) on the distribution of the aggregate interference power. Then, results obtained from this study is applied to the context of spectrum sharing. The contents of this thesis can be divided into four parts. The first part provides literature review on relevant works and some background information on related topics. The second part presents preliminary results produced at the early stages of this research which characterizes the aggregate interference based on its first two moments, and then investigates spectrum sharing based on some upper bounds like the Chebyshev's inequality. A more rigorous characterization of the aggregate interference power is presented in the third part. Then, these results are utilized to investigate spectrum sharing in large secondary networks.

\subsection{Contributions}

The following list summarizes the main contributions of this dissertation.

- We introduce a simple yet comprehensive method to determine the cumulants of the aggregate interference power originating from a wireless network. This method is quite general and applicable for finite and infinite network sizes, 
and it is flexible to encompass different system and propagation parameters such as large-scale fading, small-scale fading, or even composite fading. We also investigate the behavior of these cumulants with respect to changes in the network size and fading distributions. Moreover, we discuss how cumulants can be used to approximate the distribution of the aggregate interference power.

- We cast observations scattered in the literature about the Gaussianity of the aggregate interference power of large wireless networks in a single mathematical framework. We express the conditions for which the Gaussian approximation will be valid for the aggregate interference power generated by a Poisson field of interferers. Furthermore, we discuss the effect of different system and channel parameters on the convergence of the distribution of the aggregate interference to a Gaussian distribution.

- We study the effect of the field size on spectrum sharing opportunities. We verify that asymptotic results obtained for infinite fields are applicable for finite but relatively large fields (when the radial depth of the field is much greater than the minimum distance to the primary user) as well. We also demonstrate that in some cases, however, asymptotic results are too pessimistic hiding some spectrum sharing opportunities. Moreover, the dissertation shows that in certain situations a small reduction in the field size may create spectrum sharing opportunities while in certain other situations a huge increase in the field size may not eliminate spectrum sharing opportunities. Our results also suggest the possibility of a secondary network to concurrently share the spectrum with a primary user without the need for spectrum sensing techniques or other cognitive radio functionalities.

- We identify the smallest portion (dominant region) of the secondary network that would impact spectrum sharing opportunities. Our results show that the 
dominant region is not necessarily a small region encompassing a few interferers within the proximity of the primary user. Far interferers may tangibly contribute to spectrum sharing decisions when a higher approximation accuracy is required or when a wide exclusion region is considered. On the other hand, the dominant region shrinks with the increase in the path-loss exponent or in the level of the interference threshold specified by the primary user or a regulator. Some implications of these results are highlighted. Moreover, the results are anticipated to inspire new ideas for designing MAC protocols for secondary networks.

\subsection{Published, Submitted, and Proposed Manuscripts}

This section highlights a list of manuscripts that have been produced out of the research contained in this thesis. Status of these manuscripts at the time of writing this thesis is also indicated.

- M. Aljuaid and H. Yanikomeroglu, "On the asymptotic analysis of average interference power generated by a wireless sensor network," in Proc. IEEE Vehicular Technology Conference (VTC) 2008-Fall, Calgary, AB, Canada, Sep. 2008 [7]. This manuscript reports some initial results obtained at the early stages of our research. These initial results are shown in Section 4.1.

- M. Aljuaid and H. Yanikomeroglu, "Impact of secondary users field size on spectrum sharing opportunities," in Proc. IEEE Wireless Communications and Networking Conference (WCNC), Sydney, Australia, Apr. 2010 [21]. This manuscript contains results from Section 4.2.

- M. Aljuaid and H. Yanikomeroglu, "A cumulant-based characterization of the aggregate interference power in wireless networks," in Proc. IEEE Vehicular 
Technology Conference (VTC) 2010-Spring, Taipei, Taiwan, May 2010 [22]. This manuscript shows some of the results presented in Chapters 5.

- M. Aljuaid and H. Yanikomeroglu, "A cumulant-based investigation of the impact of secondary users' field size on spectrum sharing opportunities," submitted to IEEE Transactions on Vehicular Technology (submission: 12 April 2010) [23]. This manuscript contains results shown in Chapters 5.

- M. Aljuaid and H. Yanikomeroglu, "Investigating the validity of a Gaussian approximation for the distribution of the aggregate interference power in large wireless networks," in Proc. 25th Biennial Symposium on Communications (QBSC 2010), Queens University, Kingston, Ontario, Canada, May 2010 [24]. This manuscript contains some of the results presented in Chapter 6 .

- M. Aljuaid and H. Yanikomeroglu, "Investigating the Gaussian convergence of the distribution of the aggregate interference power in large wireless networks," accepted to IEEE Transactions on Vehicular Technology (20 April 2010) [25]. This manuscript contains results from Chapter 6.

- M. Aljuaid and H. Yanikomeroglu, "Identifying boundaries of dominant regions dictating spectrum sharing opportunities for large secondary networks," accepted to IEEE International Symposium on Personal, Indoor and Mobile Radio Communications (PIMRC) 2010, Istanbul, Turkey, Sep. 2010 [26]. This manuscript contains results shown in Chapter 7 .

- M. Aljuaid and H. Yanikomeroglu, "Impact of Secondary Network Partitioning on Aggregate Interference and Spectrum Sharing," IEEE journal manuscript in preparation. This manuscript is to contain and extend results from Chapter 7 . 


\subsection{Organization}

The rest of this thesis is organized as follows. Chapter 2 presents a literature review, and some mathematical and statistical preliminaries. The system model is described in Chapter 3. Chapter 4 highlights preliminary results developed for the effect of network size on spectrum sharing opportunities. A cumulant-based characterization and approximation of the distribution of the aggregate interference power are discussed in Chapter 5. This chapter also revisits the effect of the spatial size of the secondary network on the spectrum sharing opportunities, which is introduced in Chapter 4, but this time based on the approximation of the distribution of the aggregate interference power. The Gaussianity of the distribution of the aggregate interference power is investigated in Chapter 6. Then, Chapter 7 identifies the boundary of the dominant interference region in large secondary networks, which dictates the spectrum sharing opportunities. Finally, concluding remark and some suggestions for future work are highlighted in Chapter 8. 


\section{Chapter 2}

\section{Background and Literature Review}

This chapter provides some background information on spectrum sharing related topics including different spectrum sharing schemes and metrics used to identify the availability of spectrum sharing opportunities. Moreover, this chapter summarizes related works that deal with interference characterization and spectrum sharing in large wireless networks. The last section of this chapter highlights some important statistical and mathematical preliminaries used in the analysis and discussions in subsequent chapters.

\subsection{Spectrum Sharing}

The success in wireless technologies and applications has led to an exponential growth in the number of wireless systems and devices. These systems and devices require radio channels (bands of the radio spectrum) to communicate. However, to avoid interference between these systems, each band used to be assigned to a licensee in an exclusive manner where other systems are not allowed to access this band. Looking at a frequency allocation chart such as [27], it becomes obvious that there's not enough room in the radio spectrum to accommodate the requirements of the exponential growth in wireless systems and devices. Therefore, frequency regulators have considered changing how they manage this scarce resource, i.e., the radio spectrum. 
The Federal Communications Commission (FCC) proposal on spectrum sharing [4] has stimulated significant interest in academia and industry due to its potential for reducing the effect of radio spectrum scarcity. In the spectrum sharing proposal, a secondary user (likely an unlicensed user) could share the spectrum with a primary user (licensed user) provided that the operation of the secondary user does not introduce "harmful interference" towards the primary user $[4,5]$.

Advancements in wireless technologies pave the way for this paradigm shift in spectrum management. An interesting technology is proposed in $[28,29]$, which is considered to be the enabler for the dynamic spectrum sharing. This technology is called Cognitive Radio (CR). According to [5], CR can be defined as "an intelligent wireless communication system that is aware of its surrounding environment (i.e., outside world), and uses the methodology of understanding-by-building to learn from the environment and adapt its internal states to statistical variations in the incoming RF stimuli by making corresponding changes in certain operating parameters (e.g., transmit-power, carrier-frequency, and modulation strategy) in real-time, with two primary objectives in mind:

- highly reliable communications whenever and wherever needed;

- efficient utilization of the radio spectrum".

Some measurements campaigns were carried out to investigate the current utilization of the radio spectrum. For example, the utilization for the bands below $3 \mathrm{GHz}$ was measured at six locations including New York City [30-35]. The measurements results show that the maximum frequency utilization was $13.1 \%$ which was measured in New York City. The average of the frequency utilization for the six locations was $5.2 \%$. These measurements reflect some of the benefits and opportunities that spectrum sharing and CR could bring to the industry of wireless communications.

Important practical steps have been taken over the last few years towards the 
incorporation and implementation of $\mathrm{CR}$ and spectrum sharing concepts [36]. For example, frequency regulators have taken forward steps changing the practice of managing radio spectrum $[18,37,38]$. Moreover, some standardization bodies have formed groups mandated to establish spectrum sharing related standards, e.g., [39] [40-42]. For example, one of the important standardization activities related to spectrum sharing and CR is the IEEE Standards Coordinating Committee 41 (IEEE SCC41). The IEEE SCC41 has different groups focusing on many aspects of spectrum sharing including the following [42]:

- definitions and concepts for dynamic spectrum access,

- recommended practice for interference analysis,

- network architecture,

- policy language, and

- spectrum sensing interfaces and data structures.

Another important standardization activity is the IEEE 802.22 which aims to standardize the utilization of $\mathrm{CR}$ techniques to establish wireless regional area networks using the unused TV bands [39]. The Ecma, an industry association focusing on the standardization of the information and communication technologies, has a standard know as (ECMA-392) that specifies a physical layer and a medium access sub-layer for wireless devices willing to use the unused TV bands [43]. Moreover, there are other IEEE standards, e.g., 802.11 and 802.16, that incorporate some of the CR functionalities [42]. Furthermore, other standardization bodies such as ITU, SDR Forum, and 3GPP have some activities related to the CR [42]. 


\subsubsection{Spectrum Sharing Schemes}

There are different schemes that SUs can utilize to share the spectrum of PUs $[44,45]$. One of these schemes is based on the capabilities of SUs to identify a spectrum hole where a PU is not using that portion of the spectrum. The SU could use that portion during the absence of the PU. However, when the PU returns to that the band the $\mathrm{SU}$ should evacuate the band. This scheme is known in some literature as the overlay scheme [9]. Another scheme is when the SU could concurrently access the band of the PU even during the presence of the PU provided that the activities of SU do not cause intolerable interference (or harmful interference) towards the PU. This scheme is called the underlay scheme and mainly proposed for spread-spectrum-based SUs [9]. In a third scheme, a SU dedicates a portion of its power to relay the signal of the PU to compensate for the interference that will be introduced due to the $\mathrm{SU}$ transmission [46].

\subsubsection{Metrics for Harmful Interference}

Some metrics have been proposed in the literature to identify whether the interference generated by secondary users reaches to a level of being "harmful" to the primary users. Therefore, these metrics indicate whether the SUs could share/access the spectrum of PUs. Examples of these metrics include the following: interference temperature [47], outage probability [9], interference indicator [18], spectral outage probability [17], and interference probability $[12,14]$. In this thesis, we choose interference probability as the harmful interference metric. An explanation of this metric and justifications of our choice are discussed in the system model, i.e., Chapter 3. 


\section{$2.2 \quad$ Related work}

\subsubsection{Spectrum Sharing in Large Wireless Networks}

There could be a single SU trying to access the spectrum, or a group of SUs composing a secondary network. Works such as $[9,10,12,14,48-50]$ study the spectrum sharing in large wireless networks. The authors in [50] consider the spectrum sharing in large wireless networks. In their setup they assume that the nodes are uniformly distributed over an annulus. They assume that the individual interference power received by the PU at the center is affected by a distance-dependent attenuation and a shadow fading. The authors formulate the CDF of the individual interference power. However, they indicate the difficulty to characterize the aggregate interference. Therefore, their study of the aggregate interference is based on simulations. They also indicate that the simple lognormal approximation of the distribution of the aggregate interference is inaccurate and more complex models are required. They compared the performance of previously proposed interference management schemes. The first one is called radio environment map (REM) [51]. In this scheme, the characterization of the interference from all interferers is assumed to be known and ordered. A subset of these interferers will be chosen to meet a signal-to-noise and interference ratio (SINR) constraint. The second scheme is referred as a primary exclusion zone (PEZ) [49]. In this scheme, the minimum radius of the exclusion zone (within which no SUs are allowed to transmit) is determined based on a specified SINR. The authors highlight that REM outperforms PEZ but it requires a considerable overhead.

The authors in [14] investigate characterization of the aggregate interference from nodes deployed in an infinite plane according to a Poisson point process. A characteristic function of the aggregate interference signal is provided which reveals that the distribution of the aggregate interference signal is a symmetric stable distribution. Moreover, they indicate that the distribution of aggregate interference power is 
a skewed stable distribution. The system model in [14] considers both shadow fading and fast fading. The authors derived an expression for the characteristic function of the aggregate interference when the SUs implement spectrum sensing for the presence of PUs but in an integral form. Then, the interference probability can be evaluated numerically.

The analysis in [49] considers a system of a single primary transmitter, group of primary receivers and many SUs. The primary transmitter is located at the origin surrounded by an exclusion region where intended primary receivers are located within. No SU is allowed to transmit within the exclusion region. The exclusion region is chosen to be centered at the location of the primary transmitter not the receiver based on the argument that it is easier to detect the PU transmitter than the PU receiver especially if the receiver is passive like TV receivers. The discussion and results of the paper were based on the average of the aggregate interference power received by a primary receiver. The secondary users are assumed to be uniformly deployed over the region outside the exclusion region. The paper does not consider the effect of fading. Through some bounds, the paper tries to find the radius of the exclusion region that satisfies an outage constraint.

The authors in [12] assume that a secondary network deployed over an infinite plane in the two-dimensional space according to a PPP. The system model excludes a small disk of radius 1 meter around a primary user located at the origin. The exclusion of this disk is to avoid the singularity of the path loss model. The SUs run a spectrum sensing technique to avoid transmission if they hear a beacon sent by the primary receiver. This sensing should impose an exclusion region around the PU-RX. The characteristic function of the aggregate interference power is derived under these assumptions. Since there is no closed-form expression for CDF or the PDF of the aggregate interference power, the authors resort to deriving the cumulants. These cumulants are used to approximate the distribution of the aggregate interference power. 
The formulations count for shadow fading and multipath fading. The interference (outage) probability is also used in [12] as the harmful interference metric.

The impact of spectrum sharing on a legacy system is studied in [48]. The study is based on the assumption of an infinite secondary network sharing the spectrum with a primary user at the origin. The secondary users are spatially distributed according to a PPP. The cumulants of the aggregate interference power are provided. The authors in [48] approximate the distribution of the aggregate interference power by a lognormal random variable. The paper also investigates the impact of the absence of perfect information about the legacy system, and the impact of shadow fading. The spectrum sharing is assumed to be performed by an overlay scheme (interference avoidance), an underlay scheme (interference averaging) or a hybrid scheme. The overlay scheme outperforms the underlay scheme since it excludes the dominant interferers by creating an exclusion region. However, the hybrid scheme outperforms the overlay scheme because it averages the interference from far interferers in addition to excluding the dominant ones.

\subsubsection{Characterization of Interference in Large Wireless Networks}

The importance of characterizing the aggregate interference generated by a wireless network has some history dating back at least to packet radios [52-56]. This importance has increased with the emergence of different types of wireless networks over the past two decades, e.g., CDMA [11,13], ad-hoc and sensor networks [15, 17,20,57-61], spectrum sharing and cognitive radio networks [9,10,12,48-50,62-64]. Further history and references are provided in $[3,14]$.

The spatial distribution of nodes (interferers) in these networks has an impact on the characterization of the aggregate interference. The location and number of these nodes could be modeled by a deterministically or stochastically spatial distribution. Deterministic distributions of the nodes are applicable if the exact locations 
and number of these nodes are known or if the nodes are deployed according to a specific structure. However, it is more practical to model the distribution of the nodes stochastically. The most common stochastic model used for the spatial distribution of the nodes in wireless networks is the Poisson point process (PPP), see for example $[11,12,14,15,17,19,20,48,53,54,57,62,62,65-67]$.

Another important factor affecting the characterization of the aggregate interference is the distance-dependant attenuation model. We may categorize the relevant models to our discussion into two categories: singular (unbounded) and non-singular (bounded) models. In singular models, the function that is used to reflect the distancedependant attenuation goes to infinity when the distance between the victim receiver and the interferer is zero. On the other hand, the non-singular models avoid this singularity by not allowing the interferer to come very close to the victim receiver, or by using a function that does not have a singularity at zero.

Works such as $[14,53,54,65]$ characterize the aggregate interference of a wireless network assuming that the spatial distribution of interferers follows a PPP. These works use singular distance-dependent attenuation models. Under these assumptions, the characteristic function of the aggregate interference is obtained in a closed-form expression. The characteristic function reflects that the distribution of the aggregate interference is an alpha-stable distribution. While the characteristic function is in a closed-form expression, it is not possible, however, to convert it to a cumulative distribution function or probability density function except through numerical integrations or series expansions. There is one special case which exhibits a closed-form expression for the $\mathrm{CDF} / \mathrm{PFD}$ that is when alpha is $1 / 2$ which corresponds to having a path loss exponent of 4 [53]. It is worth noting that the $m$ th moment of alpha-stable is defined only if $m$ is less than or equal to alpha (which equals 2 divided by the path loss exponent) [68].

Other works investigate the aggregate interference in wireless networks using PPP 
and non-singular models. These works follow different approaches to characterize the aggregate interference. Some of these works focus on the mean of the aggregate interference, e.g., [8, 49]. General expressions for the moments are given in [62]. Other works such as $[10-12,48]$ derive an expression for the characteristic function and then use this function to derive cumulants of the aggregate interference. Then, approximations of the distribution of the aggregate interference can be established based on these cumulants.

Finally, there are many important papers in the literature dealing with the aggregate (co-channel) interference generated by the transmissions from many interferers. In addition, there are other papers dealing with research problems related to the sum of Rayleigh random variables, Rician random variables, or lognormal random variables. Results in these papers can be utilized to characterize the aggregate interference. However, these works don't consider the spatial distribution of the interferers or they consider the number of interferers deterministic. Examples of these works include [69-76]. In this thesis, we focus on the works that incorporate randomness in the location of the interferers, and randomness in the number of these interferers. Actually, we mainly focus on works that are based on a PPP to model the number and spatial distribution of the interferers, i.e., SUs.

\subsection{Statistical and Mathematical Preliminaries}

These sections provides some background information on statistical and mathematical topics that are used in the analysis and discussions presented in this thesis.

\subsubsection{Poisson Point Processes $[1-3]$}

A point process is a random collection of points in a space. An important application of these processes in the context of this thesis is to model the spatial distribution of 
the nodes (SUs) in Euclidean space $\mathbb{R}^{d}{ }^{1}$ A simple but an important type of these point processes is the Poisson point process. A point process is called a Poisson point process if it satisfies the following two conditions:

- If $\mathcal{R}$ is a subset of $\mathbb{R}^{d}$, the number of points (nodes) in $\mathcal{R}$ is a Poisson random variable.

- If $\mathcal{R}_{1}, \mathcal{R}_{2}, \ldots, \mathcal{R}_{k}$ are disjoint subsets of $\mathbb{R}^{d}$, random variables corresponding to the number of nodes in each subset are independent.

There is an important theorem related to the PPP that will be used later in the thesis to derive the characteristic function of the distribution of the aggregate interference power. This theorem is known as Campbell's theorem. Before introducing the theorem, it is helpful to explain a mean measure $(\wp)$ of a PPP. The mean measure of a subset, e.g., $\mathcal{R}$, equals to the average number of points in this subset, i.e., $\wp(\mathcal{R})=\mathbb{E}[\mathfrak{N}(\mathcal{R})]$, where $\mathfrak{N}(\mathcal{R})$ denotes the number of points in $\mathcal{R}$. For a PPP with a constant intensity $(\lambda)$, the mean measure of a subset equals $\lambda$ multiplied by the Lebesgue measure of the subset, e.g., the area of $\mathcal{R}$ in a two-decisional Euclidean space.

Theorem 2.3.1 (Campbell's Theorem [1]).

Let II be a Poisson point process on a space $\mathbb{S}$ with a mean measure $\wp$, and let $h$ : $\mathbb{S} \rightarrow \mathbb{R}$ be measurable where $\mathbb{R}$ is the real line. Then,

$$
\Sigma=\sum_{X \in \Pi} h(X)
$$

\footnotetext{
${ }^{1}$ Most of the discussions in subsequent chapters consider a two-dimensional Euclidean space. However, some of the results can be easily generalized to a $d$-dimensional space as noted in Appendix C.
} 
is absolutely convergent with probability 1 if and only if

$$
\int_{\mathbb{S}} \min (|h(X)|, 1) \wp(d x)<\infty
$$

If this condition holds, then for any complex $z$ for which the integral on the right converges

$$
\mathbb{E}\left[e^{z \Sigma}\right]=\exp \left[\int_{\mathbb{S}}\left(e^{z h(x)}-1\right) \wp(d x)\right]
$$

Proof.

The proof can be found in [1].

Following are some important attributes of a PPP:

- A PPP is stationary if it is invariant to translation.

- A PPP is homogeneous if its intensity, i.e., $\lambda$ is constant over the space. A homogeneous PPP is stationary.

- A PPP is isotropic if it is invariant to rotation. A homogeneous PPP is isotropic.

\subsubsection{Cumulants}

The $m$ th cumulant of a random variable $X$ whose characteristic function is $\phi_{X}(w)$ can be obtained by $[77]$

$$
\kappa_{m}=\frac{1}{j^{m}}\left[\frac{d^{m} \ln (\phi(\omega))}{d \omega^{m}}\right]_{\omega=0} .
$$

Cumulants have some properties that make them attractive for the problem considered in this thesis. Among these properties are the following:

- The $m$ th cumulant of the sum of two independent random variables is equal to the sum of the individual $m$ th cumulants of these independent random variables, 
i.e., if $Z=X+Y$ where $X$ and $Y$ are independent random variables, and $\kappa_{m}(X)$ and $\kappa_{m}(Y)$ are the $m$ th cumulants of $X$ and $Y$, respectively, then $\kappa_{m}(Z)=\kappa_{m}(X)+\kappa_{m}(Y)$ where $\kappa_{m}(Z)$ is the $m$ th cumulant of $Z$ [77].

- If $Y=c X+b$ where $Y$ and $X$ are random variables, and $c$ and $b$ are constants, then [78] $\kappa_{1}(Y)=c \kappa_{1}(X)+b, \kappa_{m}(Y)=c^{m} \kappa_{m}(X)$, for $m \geq 2$.

- As a result of the previous property, if $Y=\frac{X-\tilde{\mu}}{\sigma}$ where $\tilde{\mu}$ and $\sigma$ are the mean and the standard deviation of the random variable $X$, respectively, then $\kappa_{1}(Y)=0$, and $\kappa_{m}(Y)=\kappa_{m}(X) \sigma^{-m}=\kappa_{m}(X) \kappa_{2}(X)^{-m / 2}$, for $m \geq 2[78]$.

- Cumulants can be used to obtain some important measures of the distribution, like mean $\left(\kappa_{1}\right)$, variance $\left(\kappa_{2}\right)$, skewness $\left(\kappa_{3} \kappa_{2}^{-3 / 2}\right)$ and kurtosis excess $\left(\kappa_{4} \kappa_{2}^{-2}\right)$ [78].

- The cumulants of a random variable are closely related to its moments. For example, $\kappa_{1}=\tilde{\mu}_{1}, \kappa_{2}=\mu_{2}=\sigma^{2}, \kappa_{3}=\mu_{3}$, and $\kappa_{4}=\mu_{4}-3 \mu_{2}^{2}$ where $\tilde{\mu}_{m}$ is the $m$ th raw moment, $\mu_{m}$ is $m$ th central moment, and $\sigma^{2}$ is the variance [79] . Expressions relating cumulants to central or raw moments are provided in [78].

- Cumulants of a random variable can be used to approximate the distribution function of that random variable through some approaches like an Edgeworth series expansion [11]. Alternatively, cumulants can be used to determine the moments, and then moments-based approximations can be applied $[78,80]$. 


\section{Chapter 3}

\section{System Model}

This chapter describes the system model that we use to develop the results presented in the rest of this thesis.

\subsection{Spatial Distribution of Secondary Users}

The analysis in this thesis is based on modeling a secondary network as a twodimensional field of interferers deployed over a region of area $A$ with an annular sector shape (ring or disk shapes are special cases). The set of active secondary user transmitters (SU-TXs) is assumed to follow a Poisson point process with a homogeneous density $\lambda$. Based on this assumption, the number of SU-TXs in a region is a Poisson random variable with a parameter specified by the multiplication of $\lambda$ by the area of that region. Moreover, the number of SU-TXs in disjoint regions are independent Poisson random variables [1]. The field of SU-TXs is assumed to have an inner radius of $r_{o}$. We call exclusion region the disk $b\left(O, r_{o}\right)$ of radius $r_{o}$ and centered at the origin. The field has a radial depth of $L$, making the outer radius of the field $r_{o}+L$. The field spans over an angle of $\theta$ as seen by the victim receiver, i.e., a primary user receiver (PU-RX), at the origin, as illustrated in Fig. 3.1. 


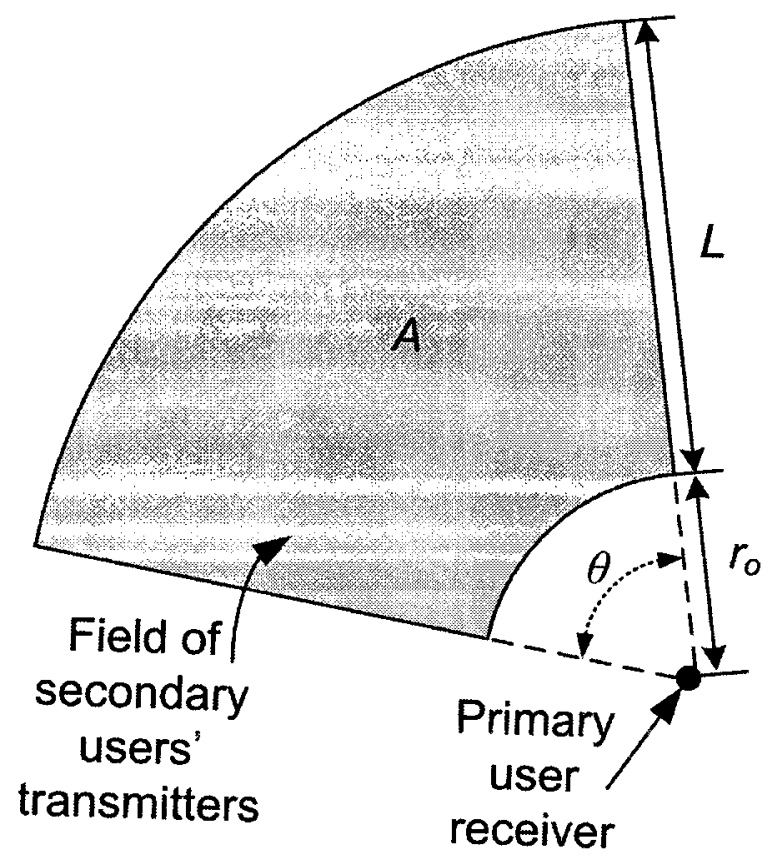

Figure 3.1: Field layout.

\subsection{Aggregate Interference Power}

The individual interference power received by a PU-RX at the origin due to the transmission of node $i$ is denoted by $I_{i}$. Under the assumption of incoherent addition of interfering signals, the aggregate interference power received by the PU-RX can be expressed as [81]

$$
I_{A}=\sum_{i \in \Lambda} I_{i}
$$

where $\Lambda$ is a set of active SU-TXs. $I_{i}$ can be modeled as

$$
I_{i}=X_{i} g\left(r_{i}\right)
$$

where $X_{i}$ is a positive random variable that can be modeled as the multiplication of deterministic quantities and various random variables reflecting the transmitter 
power, antenna gain, channel attenuation (including multipath and shadow fading) and other factors [82]. In a mathematical representation, $X_{i}$ can be modeled as

$$
X_{i}=\prod_{l} X_{i, l}
$$

where the function $g\left(r_{i}\right)$ represents a path-loss model (or more precisely the distancedependent attenuation), which is discussed in another subsection. $X_{i, l}$ is a deterministic or a random variable. A similar representation is used in [14].

Equation (3.3) is general enough to account for adjacent-channel interference. The overlap between the frequency bands used by SU-TX $i$ and PU-RX can be captured by one of $X_{i, j}$, e.g., $X_{i, 0}$. This $X_{i, 0}$ has a range that goes from 0 to 1 ; it is equal to 1 if the frequency band of the interfering node is totally within the frequency band of the primary user, whereas the $X_{i, 0}$ is equal to zero if the frequency band of the interference is outside the frequency band of the primary user and does not cause an adjacent channel interference. To illustrate how the adjacent-channel interference can be incorporated in the formulations, let us assume that an interferer SU-TX $i$ introduce adjacent channel interference. Let the transmit power of this SU-TX $i$ be $P$, the distance between this SU-TX $i$ and PU-RX be $r_{i}$, and the channel be a non-fading channel. Other parameters can be ignored to simplify the illustration of the effect of the adjacent-channel interference. Therefore, $X_{i}$ can be expressed as $X_{i}=X_{i, 0} X_{i, 1}$ where $X_{i, 1}=P$ and $X_{i, 0}$ reflects the fraction of the power $P$ that leaks to the channel of the PU-RX. Assuming $X_{i, 1}=0.1$, the interference experienced by the PU-RX due to the transmission of SU-TX $i$ can be expressed as $I_{i}=0.1 g\left(r_{i}\right) P$. Under the assumption of incoherent addition of interfering signals, the value of this $I_{i}$ should be added to the interference power generated by other active SU-TXs according to (3.1) for the evaluation of the aggregate interference power. In this thesis, $X_{i, 0}$ is considered to be 1 which corresponds to the worst case. The results reported in this 
thesis can easily be extended to consider the cases where $X_{i, 0}<1$.

In analyzing the aggregate interference of a Poisson field, it is common to assume that $X_{i}$ s are independent and identically distributed (i.i.d.) random variables $[9,12$, $14,54]$. In this thesis, we follow the same assumption.

\subsection{Path-Loss Models}

The most common path-loss model used in literature is

$$
g\left(r_{i}\right)=k r_{i}^{-n}, r_{i} \geq 0
$$

where $k$ is a constant, $r_{i}$ is the distance between the SU-TX $i$ and the PU-RX, and $n$ is the path-loss exponent. This model is commonly used in contexts similar to the one we consider in this thesis due to its mathematical tractability [83]. However, this model suffers from a singularity at $r_{i}=0$. Therefore, it is known as a singular (or unbounded) path-loss model $[16,83]$.

There are non-singular (bounded) path-loss models used in the literature as well, e.g., $[12,16,62,84]$. These non-singular models are similar, with some variations. Following is an example:

$$
g\left(r_{i}\right)= \begin{cases}k r_{i}^{-n}, & r_{i} \geq r_{c} \\ k r_{c}^{-n}, & r_{i}<r_{c}\end{cases}
$$

where the quantity $k r_{c}^{-n}$ is constant, and $r_{c}>0$ is the radius at which the slope of the model starts changing.

It is indicated in [16] that the selection of the path-loss model has a significant impact on the characterization of the aggregate interference. The authors in [83] investigated the effect of the unbounded model on the performance analysis of wireless networks and indicated that more realistic performance figures are obtained by using 
bounded models. We therefore consider the use of a non-singular model, specifically (3.5) with $n>2$. Without loss of generality, we take $k=1$ assuming its effect is absorbed by $X_{i}$.

\subsection{Harmful Interference Metric}

The harmful interference metric that is used in this thesis is the interference probability. This metric can be described as

$$
P\left(I_{A} \geq I_{t h}\right) \leq \beta,
$$

which means that the probability of the aggregate interference being greater than a certain interference threshold, $I_{t h}$, should not exceed $\beta$, where $\beta \ll 1$ [12]. If (3.6) is not violated, then the aggregate interference is considered to be non-harmful. We choose this metric because it has a fundamental and versatile form, which is mainly based on the complimentary cumulative distribution function (CCDF). Thus, results of this thesis should be useful even if different metrics are used provided that these metrics depend on the distribution function of $I_{A}$.

We are interested in studying how the interference probability, and hence the spectrum sharing, behave with respect to changes in the field size, mainly $L$, and the other channel and system parameters. To achieve this, the distribution of $I_{A}$ or at least some of its characteristics are required. In this thesis, we characterize the aggregate interference power using its cumulants. Background information on cumulants is included in Chapter 2. 


\subsection{Summary}

In this chapter, we described the system model that we use to obtain the results reported in the rest of the thesis. Description of the system model covered the spatial distribution of the active secondary users, the distance-dependant attenuation model and the harmful interference metric. This model incorporates different sources of randomness, such as the number of active SU-TXs, locations of these active SU-TXs, fading components in wireless channels between these active SU-TXs and the PURX. Moreover, the model can reflect random fluctuations in antenna gains and power levels. The model should be applicable also for the case when all the parameters are deterministic; however, the aggregate interference power will be deterministic and might be described by the formulations obtained in this thesis for the first cumulant (or moment). 


\section{Chapter 4}

\section{Preliminary Results on Interference}

\section{Characterization}

This chapter presents an initial study performed at the early stages of the research reported in this thesis. This chapter has two main sections. The first section discusses the first moment (or cumulant) of the aggregate interference power. The second section incorporates the variance, i.e., the second cumulant, in the discussion. Upper bounds on the interference probability are also discussed in these two sections. More advanced results are reported in subsequent chapters, which are based on an approximation of the distribution of $I_{A}$ rather than upper bounds. However, these two sections are included in the thesis for reasons highlighted in the introduction of Section 4.1 and Section 4.2 .

\subsection{Asymptotic Analysis of the Average Interference Power Generated by a Wireless Sensor Network Towards a Pri- mary User}

While this section focuses on the average of the aggregate interference power, it includes some ideas not mentioned in other chapters of the thesis. These ideas include the representation of the whole field of interferers by a single virtual interferer (node). 
Moreover, it provides some formulations for the aggregate interference power when the shape of the field can be approximated by a rectangular shape. Furthermore, it investigates the behavior of the average interference power when the path-loss exponent is equal to 2. Finally, discussions in this section are presented in the context of wireless sensor networks sharing a spectrum with a PU.

\subsubsection{Spectrum Sharing for Wireless Sensor Networks}

Massive deployments of wireless sensor networks (WSNs) are expected in the near future [85]. Since it is not a viable solution to acquire a spectrum license for a WSN due to the high cost associated with it, a WSN is likely to share a frequency band with other systems. Current WSN implementations share unlicensed frequency bands with other unlicensed systems, e.g., WiFi and Bluetooth $[86,87]$. The FCC Spectrum Policy Task Force proposed that licensed frequency bands could be shared between licensed users (primary users) and unlicensed users (secondary users) [4]. This spectrum sharing will be under the condition that the performance of primary users communication is not degraded. The spectrum sharing proposal creates a number of opportunities for unlicensed systems like WSNs and leads to the efficient use of this invaluable resource: the RF spectrum.

Some upcoming wireless technologies may empower the secondary users to dynamically share a frequency band with other primary users. For instance, the cognitive radio technology will enable the secondary users to sense the environment around them and to identify spectrum holes that can be used without affecting the performance of primary users [5]. A spectrum hole is defined in [5] as a frequency band that is underutilized by the primary user. Although the cognitive radio technology will increase the complexity of the sensor nodes [88], we envision that these complexity issues will be resolved by future advancement in hardware technologies.

Characterizing the aggregate interference power of a WSN has been investigated 
in other works, such as [59]. However, to the best of our knowledge, no work has been devoted to study the effect of the sensor field dimensions on the total interference power generated by a WSN towards another system. The focus of this section is on the behavior of the average interference power of a WSN towards a primary user with respect to the changes in the field size.

The rest of this section is organized as follows. Subsection 4.1.2 provides the initial formulation of the average interference power generated by a WSN towards another system. This subsection also introduces possible representation of a sensor field by a single virtual node producing equivalent average interference power. Subsections 4.1.3 and 4.1.4 extend the formulation developed in Subsection 4.1.2 to sensor fields with the shape of an annular sector and a rectangle, respectively. Subsections 4.1.3 and 4.1.4 also discuss the behavior of the average interference power due to the expansion

of the sensor field. Subsection 4.1.5 demonstrates the use of the annular sector and rectangular shapes in order to provide a conservative approximation of the interference generated by any sensor field with an arbitrary shape. The average interference power can be used in the well-known Markov Inequality to reach a conservative decision about the probability of interference, as shown in Subsection 4.1.5.2.

\subsubsection{Average Interference Power}

The objective of this subsection is to develop a mathematical formulation of the average interference power generated by a sensor field towards a primary user receiver (PU-RX). Let us assume that there is a wireless sensor node at a distance $r_{i}$ from PU-RX. The average interference power that would reach PU-RX from this node would be

$$
I_{i}=X_{i} g\left(r_{i}\right)
$$


Then, the total interference power can be written as

$$
I_{A}=\sum_{i=1}^{N} I_{i}=\sum_{i=1}^{N} X_{i} g\left(r_{i}\right) .
$$

So, the mean value of $I_{A}$ becomes

$$
\mathbb{E}\left[I_{A}\right]=\mathbb{E}\left[\sum_{i=1}^{N} X_{i} g\left(r_{i}\right)\right]
$$

Since a) $X_{i}$ and $r_{i}$ are assumed to be independent random variables, b) $X_{i}$ are identically distributed, and c) $r_{i}$ are also identically distributed, then (4.3) can be written as

$$
\mathbb{E}\left[I_{A}\right]=\mu_{X} \mu_{N} \mathbb{E}\left[g\left(r_{i}\right)\right]
$$

where $\mu_{X}=\mathbb{E}\left[X_{i}\right]$ and $\mu_{N}=\mathbb{E}[N]$. In this chapter, we focus on the case where $r_{o} \geq r_{c}>0$. Therefore, $\mathbb{E}\left[g\left(r_{i}\right)\right]=\mathbb{E}\left[r_{i}^{-n}\right]$.

Writing $\mathbb{E}\left[I_{A}\right]$ in terms of the average interference power coming from a node at a distance $r_{o}$, i.e., $\bar{I}_{o}=\mu_{X} r_{o}^{-n}$, results in

$$
\mathbb{E}\left[I_{A}\right]=\bar{I}_{o} r_{o}^{n} \sum_{i=1}^{N} \mathbb{E}\left[r_{i}^{-n}\right]=K \bar{I}_{o}
$$

where $K$ is a scaling factor which absorbs the spatial distribution of interfering nodes and the distance-dependent attenuation. The whole field can be represented by a single virtual node that would generate an equivalent level of average interference power received by a PU-RX, see Fig. 4.1. This virtual node concept is used here just to simplify the representation and the calculation of the average interference power of a large wireless network. Therefore, this representation should not be used for other purposes without a careful thinking about its validity for such purposes.

In order to examine the behavior of the average interference power with respect 


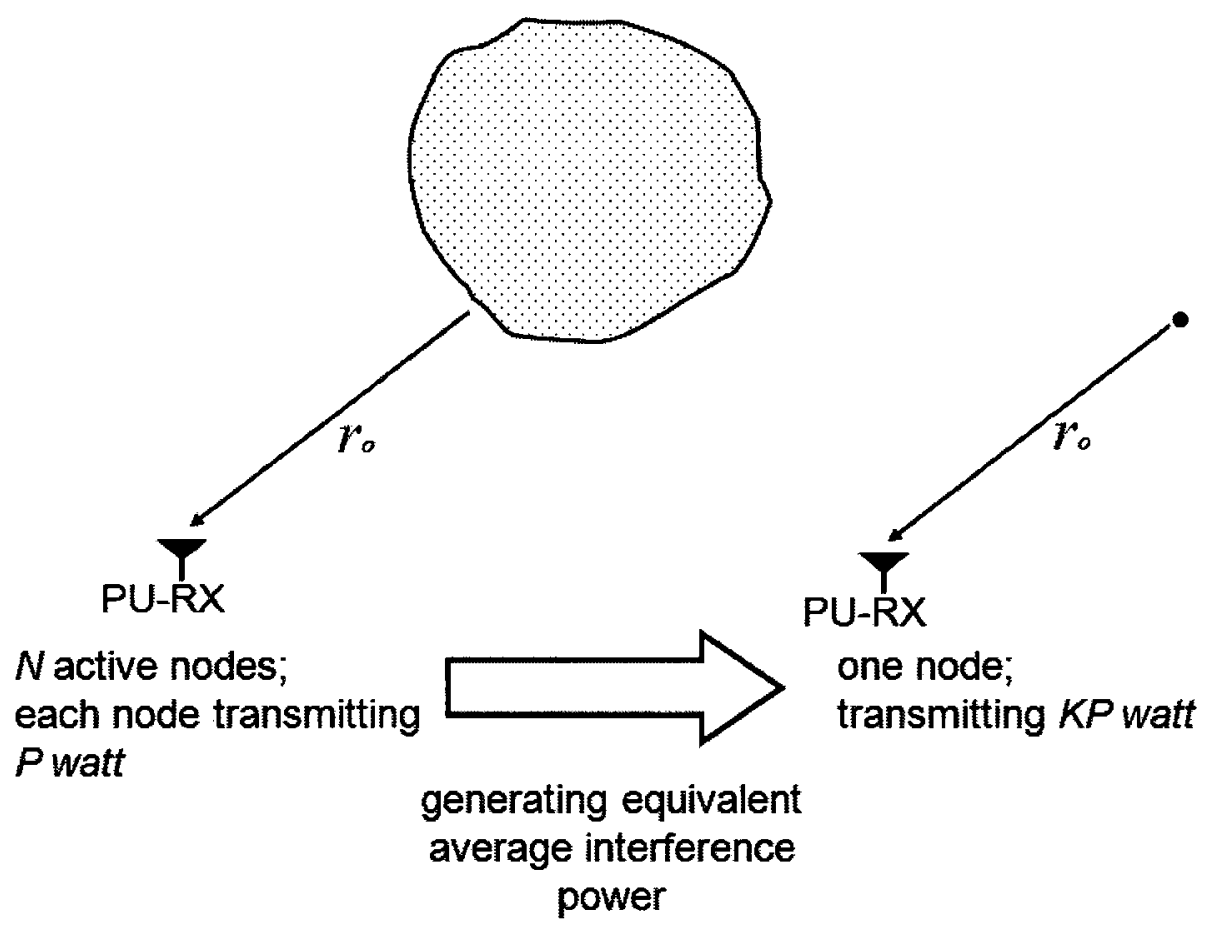

Figure 4.1: Representing a sensor field by a signal virtual node generating equivalent level of average interference power.

to the changes in field size, two rather simple but important shapes for the sensor field are considered. They are the annular sector and rectangular shapes, shown in Fig. 4.2 and Fig. 4.5, respectively, which are referred to as Shape I and Shape II for brevity. These shapes could be used to provide a conservative approximation (upper bound) of the interference power generated by a sensor field with any arbitrary shape.

In the next subsection, a formulation of $K$ is derived for a field with the annular sector shape.

\subsubsection{Shape I: Sensor Field of Annular Sector Shape}

In this subsection, we work with a secondary field with the shape of an annular sector as shown in Fig. 4.2. Since the secondary users are uniformly distributed over a finite region of the secondary network, the formulation of $K$ for this shape can be derived 


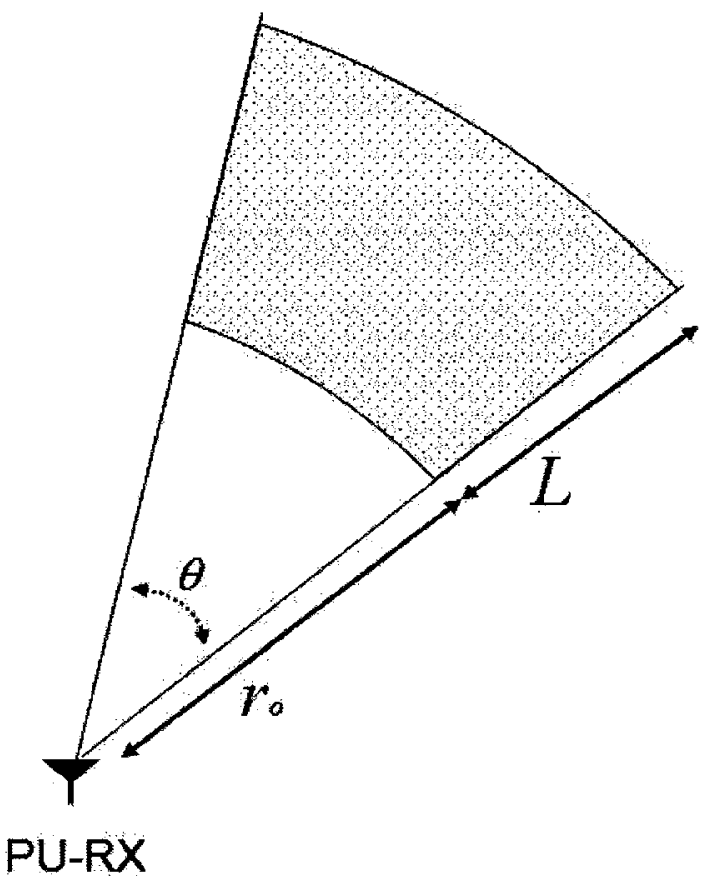

Figure 4.2: A sensor field with the shape of an annular sector.

and written as

$$
K=\left\{\begin{array}{ll}
\lambda \theta r_{o}^{2} \ln \left(1+\frac{L}{r_{o}}\right), & n=2 \\
\frac{1}{n-2} \lambda \theta r_{o}^{2}\left[1-\left(\frac{r_{o}}{r_{o}+L}\right)^{n-2}\right], & n>2
\end{array} .\right.
$$

Equation (4.6) highlights the effect of different wireless network parameters on the average interference power. For the annular sector shape, the effect of the changes in $\theta$ is obvious. Therefore, in the remaining part of this section, we focus on discussing the effect of changes in $L$ (the radial depth). It is clear from (4.6) that what matters is the ratio $L / r_{o}$ rather than merely the value of $L$. In this section, $r_{o}$ is assumed to be fixed; thus, the changes in the ratio $L / r_{o}$ are due to changes in $L$. Fig. 4.3 shows the effect of the changes in $L / r_{o}$ on the value of $K$ and, hence, on the average interference power. In this figure, the value of $K$ is normalized by $\lambda \theta r_{o}^{2}$ which represents the number of active nodes in an area of $\theta r_{o}^{2}$. This value, $\lambda \theta r_{o}^{2}$, is another important parameter in 


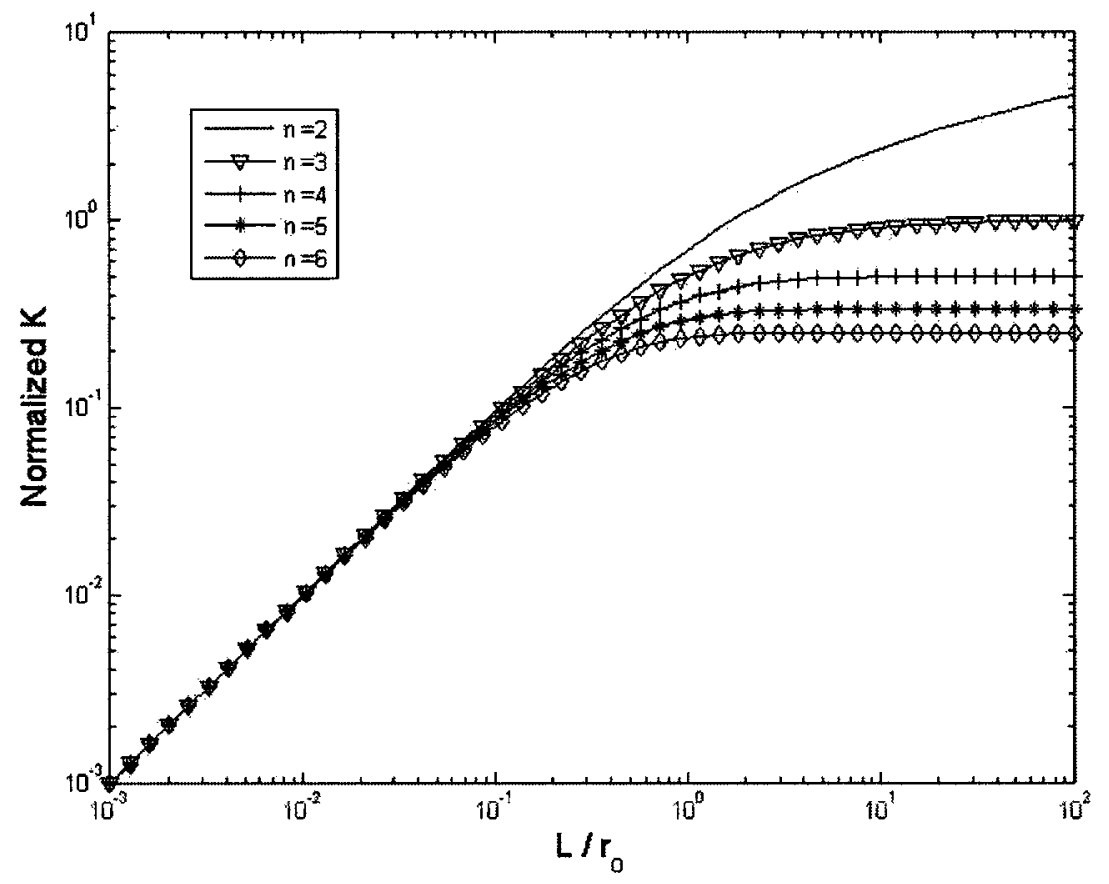

Figure 4.3: Normalized $K$, i.e, $K / \lambda r_{o}^{2}$, vs. $L / r_{o}$ for different values of $n$.

determining the value of $K$ and, hence, of the average interference power. The term $\theta r_{o}^{2}$ might be considered as a reference area $A_{\text {ref }}$ and the term $\lambda \theta r_{o}^{2}$ as a reference number of active nodes $\left(N_{\text {ref }}\right)$. With these, (4.6) can be rewritten as

$$
K=\lambda A_{\text {ref }} f\left(n, \frac{L}{r_{o}}\right)=N_{\text {ref }} f\left(n, \frac{L}{r_{o}}\right)
$$

where $f\left(n, \frac{L}{r_{o}}\right)$ is a scaling function which depends on $n$ and $L / r_{o}$,

$$
f\left(n, \frac{L}{r_{o}}\right)=\left\{\begin{array}{ll}
\ln \left(1+\frac{L}{r_{o}}\right), & n=2 \\
\frac{1}{n-2}\left[1-\frac{1}{\left(1+\frac{L}{r_{o}}\right)^{n-2}}\right], & n>2
\end{array} .\right.
$$


The value of $f\left(n, \frac{L}{r_{o}}\right)$ is bounded by 1 for $n>2$. This holds true even if $L$ goes to infinity. Therefore, the value of $K$ and, hence, the average interference power of an infinite sensor field, is bounded by a maximum value provided that the minimum distance from the field to the primary user is fixed. From (4.5) and (4.7), the maximum value of the average interference power is determined by the average interference power generated by a single node at $r_{o}$ scaled by the value of $K$. The maximum value of $K$ as shown above is the number of active nodes in a reference area, $K=\lambda \theta r_{o}^{2}$, or a fraction of it. For $n=2, f\left(n, \frac{L}{r_{o}}\right)$ and therefore $K$, and in turn the average interference, change logarithmically with the increase in $L / r_{o}$. Consequently, $f\left(n, \frac{L}{r_{o}}\right)$ can exceed 1.

A relevant but limited observation about the effect of the field size on the average interference power is made in [59]. That paper highlights the fact that an increase in the field size of a WSN does not necessarily lead to an increase in the total interference power. The observation is made for $n=3$ and $n=6$ based on simulation and numerical results. However, the paper does not provide expressions for the average interference power or the asymptotic constants. In this section, we provide exact expressions describing the behavior of the average interference generated by a sensor field towards a primary user. Expressions for the asymptotic constants for the case of $n \geq 2$ are also presented (moreover, we show that the behavior of the average interference power with respect to $L$ is different when $n=2$.)

We discuss above how the average interference would change when $L / r_{o}$ goes to infinity. On the other hand, when the field's depth $L$ is very small compared to $r_{o}$, the average interference power changes linearly with the changes in $L / r_{o}$. This holds true for any $n \geq 2$.

The asymptotic behavior of $K$ with respect to $L / r_{o}$ is shown Fig. 4.3 which can 
be summarized in a mathematical form as follows:

$$
\begin{gathered}
K \approx \lambda \theta r_{o} L ; n \geq 2 \text { and } L \ll r_{o}, \\
K \approx \begin{cases}\lambda \theta r_{o}^{2} \ln \left(\frac{L}{r_{o}}\right), & n=2 \text { and } L \gg r_{o} \\
\frac{1}{n-2} \lambda \theta r_{o}^{2}, & n>2 \text { and } L \gg r_{o}\end{cases}
\end{gathered}
$$

From the discussions so far, it has become clear that $r_{o}$ is a very important parameter in determining the average interference power. The value of $r_{o}$ controls the reference area which determines the maximum number of active nodes considered in calculating $K$ and, hence, the total average interference power. In addition, the value of $r_{o}$ sets up the different regions of how average interference power would behave with changes in $L$.

The value of $K$ can be interpreted as the number of active nodes in an effective area $\left(A_{\text {eff }}\right)$ within the sensor field. From (4.6) and $K=\lambda A_{\text {eff }}$, we can write $A_{\text {eff }}$ as

$$
A_{\mathrm{eff}}= \begin{cases}\theta r_{o}^{2} \ln \left(1+\frac{L}{r_{o}}\right), & n=2 \\ \frac{1}{n-2} \theta r_{o}^{2}\left[1-\frac{1}{\left(1+\frac{L}{r_{o}}\right)^{n-2}}\right], & n>2\end{cases}
$$

When $L \ll r_{o}$, the $A_{\text {eff }}$ is equivalent to the total area of the sensor field. On the other hand, when $L \gg r_{o}$, the value of $A_{\text {eff }}$ is much less than the total area of the sensor field. For $n>2$ and $L \gg r_{o}, A_{\text {eff }}$ is limited by a maximum value regardless of how big the sensor field is. These remarks are deduced from (4.12) and represented graphically in Fig. 4.4. 


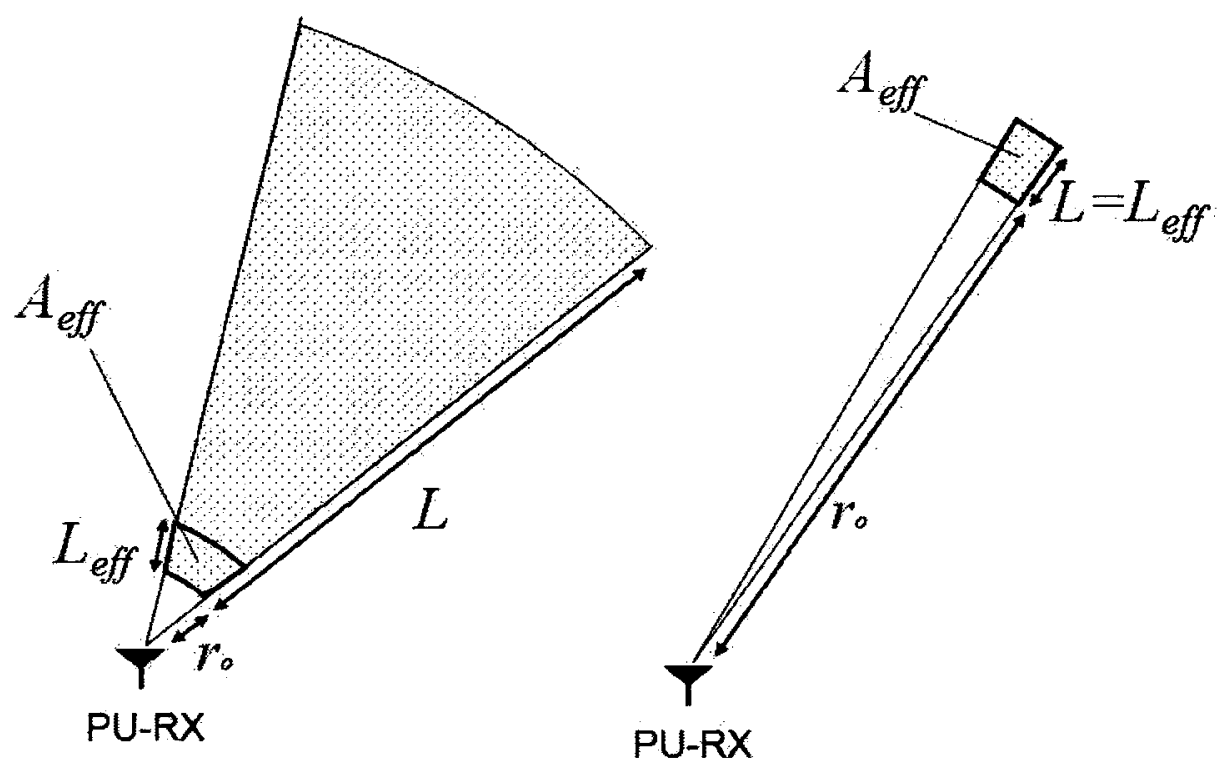

Figure 4.4: Effective areas of Shape I when $L \gg r_{o}$ and $L \ll r_{o}$.

$$
A_{\text {eff }} \approx \begin{cases}\theta r_{o} L, & n \geq 2 \text { and } L \ll r_{o} \\ \theta r_{o}^{2} \ln \left(\frac{L}{r_{o}}\right), & n=2 \text { and } L \gg r_{o} . \\ \frac{1}{n-2} \theta r_{o}^{2}, & n>2 \text { and } L \gg r_{o}\end{cases}
$$

For example, assume there is a sensor field with an $L=10 r_{o}$ and $n=3$. In this case, $A_{\text {eff }}$ would be $1 / 60$ of the total area of the sensor field.

As a summary of this subsection, the main observations are listed below: 
- The maximum value of $K$ and thus the average interference power for an unbounded sensor field is bounded. The maximum value of $K$ is limited by

$$
K \approx\left\{\begin{array}{ll}
\lambda \theta r_{o}^{2} \ln \left(\frac{L}{r_{o}}\right), & n=2 \text { and } L \gg r_{o} \\
\frac{1}{n-2} \lambda \theta r_{o}^{2}, & n>2 \text { and } L \gg r_{o}
\end{array} .\right.
$$

- For $n=2$, the value of $K$ and, hence, the average interference power, increases logarithmically with the increase in $L$.

- For $n>2$, the value of $K$ and, hence, the average interference power, asymptotically approaches to a constant level with the increase in $L$. The asymptotic constant decreases with a factor of $(n-2)$ as $n$ increases.

- For $L<<r_{o}$, the value of $K$ and, hence, the average interference power, depends linearly on $L$.

- To find the average interference power of a sensor field towards a primary user at point $X$, the following needs to be done: 1) Find the average interference power generated by a single node at a distance $r_{o}$ from PU-RX. 2) Multiply that average by the number of interfering nodes in the area of $\theta r_{o}^{2}$. 3) Scale the result by a proper scaling factor $f\left(n, L / r_{o}\right)$, which depends on $n$ and $L / r_{o}$ (refer to $(4.7))$.

\subsubsection{Shape II: Sensor Field of Rectangular Shape}

This subsection considers a sensor field with a rectangular shape as shown in Fig. 4.5a. The width of this sensor field is denoted by $W$ and the depth is denoted by $L$. 


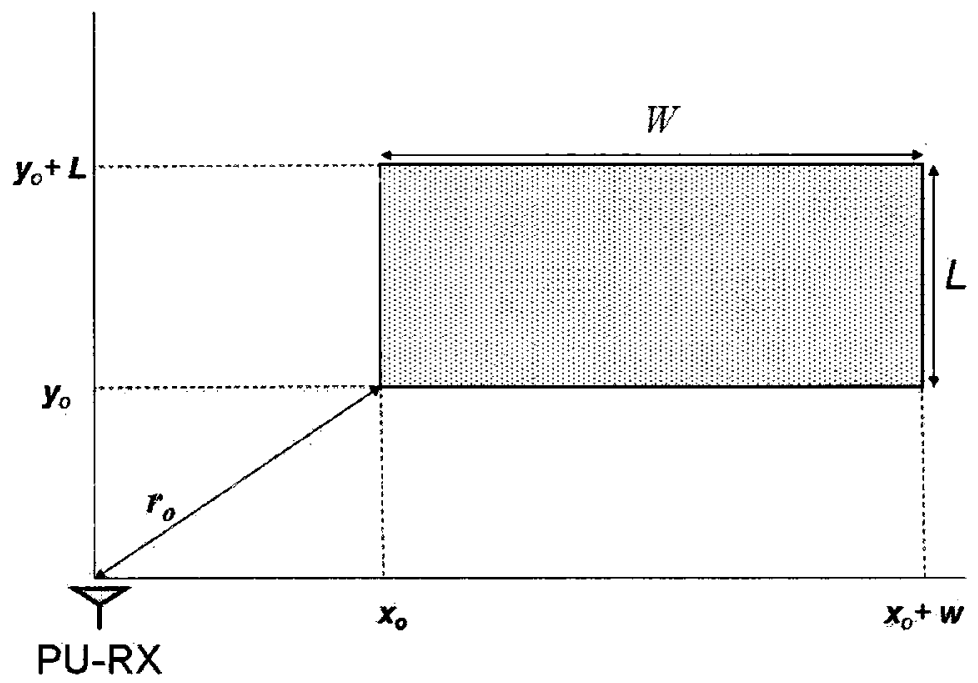

(a)

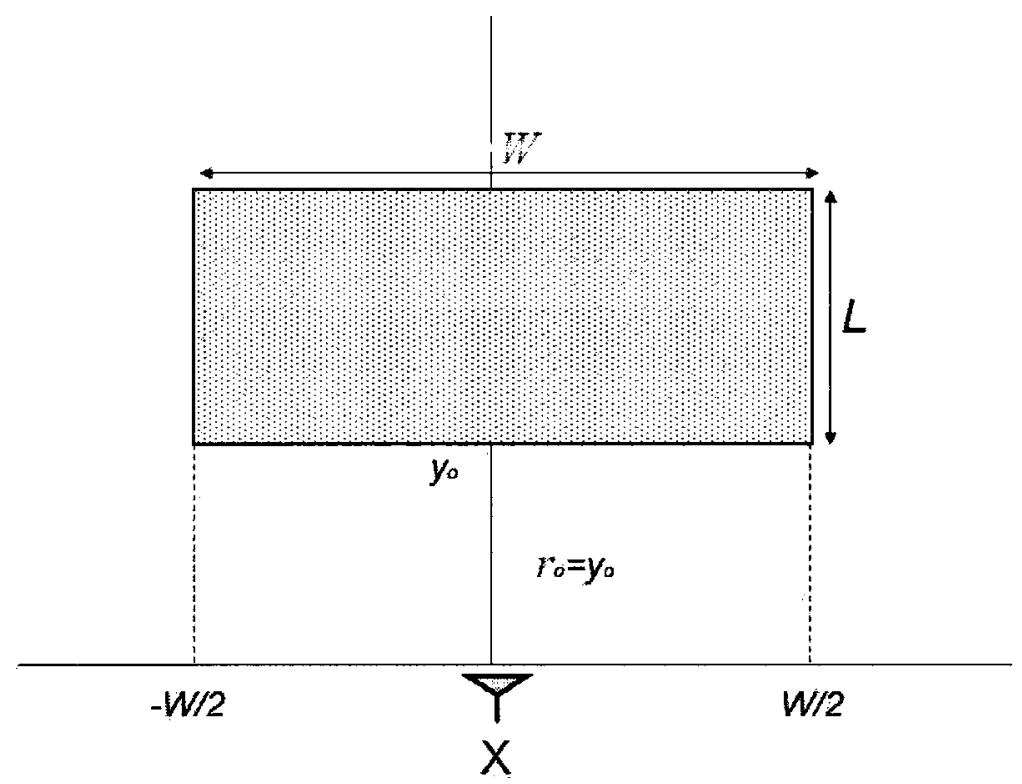

(b)

Figure 4.5: A sensor field of a rectangular shape (Shape II). (a) General alignment; (b) Worst-case alignment (given $y_{o}$ is fixed).

Assuming the nodes are uniformly distributed over this field, $K$ can be expressed as

$$
K=\lambda r_{o}^{n} \int_{y_{o}}^{y_{o}+L} \int_{x_{o}}^{x_{o}+W} \frac{1}{\left(\sqrt{x^{2}+y^{2}}\right)^{n}} d x d y
$$


which results in

$$
\begin{aligned}
K= & \frac{\lambda r_{o}^{n}}{(n-1) y_{o}^{n-2}} \times \\
& \left(\frac{x_{o}+W}{y_{o}}\left(\dot{h}\left(\frac{x_{o}+W}{y_{o}}\right)-\frac{\dot{h}\left(\frac{x_{o}+W}{y_{o}+L}\right)}{\left(1+\frac{L}{y_{o}}\right)^{n-1}}\right)-\frac{x_{o}}{y_{o}}\left(\dot{h}\left(\frac{x_{o}}{y_{o}}\right)-\frac{\dot{h}\left(\frac{x_{o}}{y_{o}+L}\right)}{\left(1+\frac{L}{y_{o}}\right)^{n-1}}\right)\right),
\end{aligned}
$$

where $h(z)$ is an abbreviated notation for the following hypergeometric function [89]

$$
{ }_{3} F_{2}\left(\frac{1}{2}, \frac{n-1}{2}, \frac{n}{2} ; \frac{3}{2}, \frac{n+1}{2} ;-z^{2}\right)=\sum_{k=0}^{\infty} \frac{\left(\frac{1}{2}\right)_{k}\left(\frac{n-1}{2}\right)_{k}\left(\frac{n}{2}\right)_{k}}{\left(\frac{3}{2}\right)_{k}\left(\frac{n+1}{2}\right)_{k}} \frac{\left(-z^{2}\right)^{k}}{k !}
$$

where $(a)_{k}=a(a+1)(a+2) \ldots(a+k-1)$ and $(a)_{0}=1$.

Equation (4.15) is applicable to any rectangular shape with arbitrary alignment. Before applying (4.15) to a sensor field with a rectangular shape, the $\mathrm{x}$-axis and $\mathrm{y}$-axis should be chosen in such a way that they intersect at the location of the primary user, and are parallel to the edges of the the sensor field.

An interesting special case for the rectangular shape field is when PU-RX is facing the center of one of the edges, for example $W$ as shown in Fig. 4.5b. This case is the worst-case scenario for the positioning of a rectangular field with respect to PU-RX, provided that $y_{o}$ is fixed. For this case, (4.15) can be simplified by substituting $x_{o}$ with $W / 2$ and $y_{o}$ with $r_{o}$ to yield

$$
K=\frac{2 \lambda r_{o}^{2}}{(n-1)}\left(\frac{1}{2} \frac{W}{r_{o}} \dot{h}\left(\frac{1}{2} \frac{W}{r_{o}}\right)-\frac{\left(\frac{1}{2} \frac{W}{r_{o}+L}\right) \dot{h}\left(\frac{1}{2} \frac{W}{r_{o}+L}\right)}{\left(1+\frac{L}{r_{o}}\right)^{n-1}}\right)
$$

Equation (4.17) can be reduced to simpler formulas for some integer values of $n$. Considering (4.17), it can be said that $K$ depends on the density of active nodes multiplied by a reference area and scaled by a factor that depends on $n, L / r_{o}$, and 
$W / r_{o}$. By choosing the reference area for the rectangular shape as $r_{o}^{2}$, and denoting the scaling factor by $g\left(n, W / r_{o}, L / r_{o}\right),(4.17)$ can be rewritten as

$$
K=\lambda r_{o}^{2} g\left(n, W / r_{o}, L / r_{o}\right)
$$

Once again, it can be seen for the rectangular shape as well that rather than the exact values of $L$ and $W$, their ratios to $r_{o}$ determine the total average interference.

Next, we would like to study the asymptotic behavior of the average interference power with respect to the changes in the field dimensions, $W$ and $L$. As shown in (4.17), the value of $K$ and, hence, the average interference power, depends on the ratio of the field dimensions to $r_{o}$. Therefore, the focus of the asymptotic discussion is on $W / r_{o}$ and $L / r_{0}$.

Equation (4.17) is plotted in Fig. 4.6 for $n=3$ to show an example of the behavior of $K$ with respect to the changes in $W / r_{o}$ and $L / r_{o}$. For a fixed value of $L / r_{o}$, the value of $K$ depends linearly on $W / r_{o}$ when $W / r_{o}<<1$. However, when $W / r_{o}>>1$ the value of $K$ approaches to a constant regardless of how big $W / r_{o}$ is. Similar behavior is observed for $K$ with respect to the changes in $L / r_{o}$.

Fig. 4.7 shows more plots for $K$ with respect to changes in $L / r_{o}$ for different values of $n$. Fig. 4.7a focuses on the case when $W / r_{o}>>1$. From Fig. 4.7a, it is clear that when $L / r_{o}<<1$, the value of $K$ depends linearly on the changes in $L / r_{o}$. On the other hand, the value of $K$ approaches to a constant when $L / r_{o}>>1$. An exception to this observation occurs when $n=2$; in this case $K$ does not approach to a constant with the increase in $L / r_{o}$. Fig. 4.7b shows the behavior of $K$ for the case when $W / r_{o}<<1$. For $L / r_{o}>>1$ in Fig. $4.7 \mathrm{~b}$, the value of $K$ approaches to a constant even for $n=2$. However, when $L / r_{o}<<1$, the value of $K$ changes linearly with the value of $L$.

As a summary, the following observations can be made: 


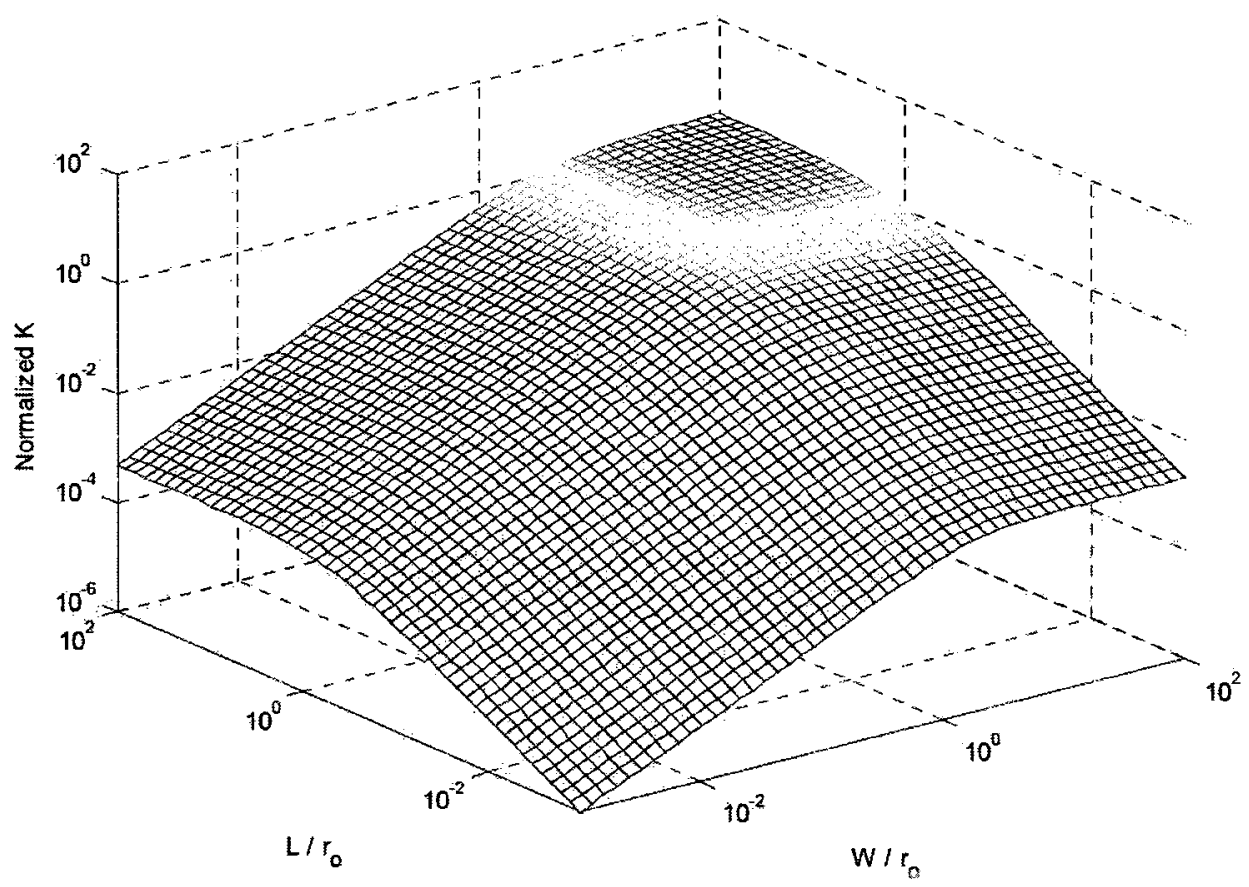

Figure 4.6: Normalized $K$, i.e, $K / \lambda r_{o}^{2}$, for Shape II for $n=3$.

- An increase in the spatial size of the sensor field does not necessarily cause an increase in the average interference power provided that the minimum distance between the sensor field and the primary user is fixed. This is obvious in the situations where the width and length of the field are large compared to $r_{o}$ provided that $n>2$.

- For a sensor field with a width $W$ much greater than $r_{o}$, a further increase in $W$ does not introduce a significant change in the average interference power even for $n=2$.

- If $L<<r_{o}$, the average interference power linearly depends on $L$. Similarly, for $W<<r_{o}$, the average interference power depends linearly on $W$.

- The quantity $\lambda r_{o}^{2}$, which reflects the number of active nodes in an area of $r_{o}^{2}$, is very important in finding the value of $K$. For $n \geq 3$, multiplying $\lambda r_{o}^{2}$ by a 


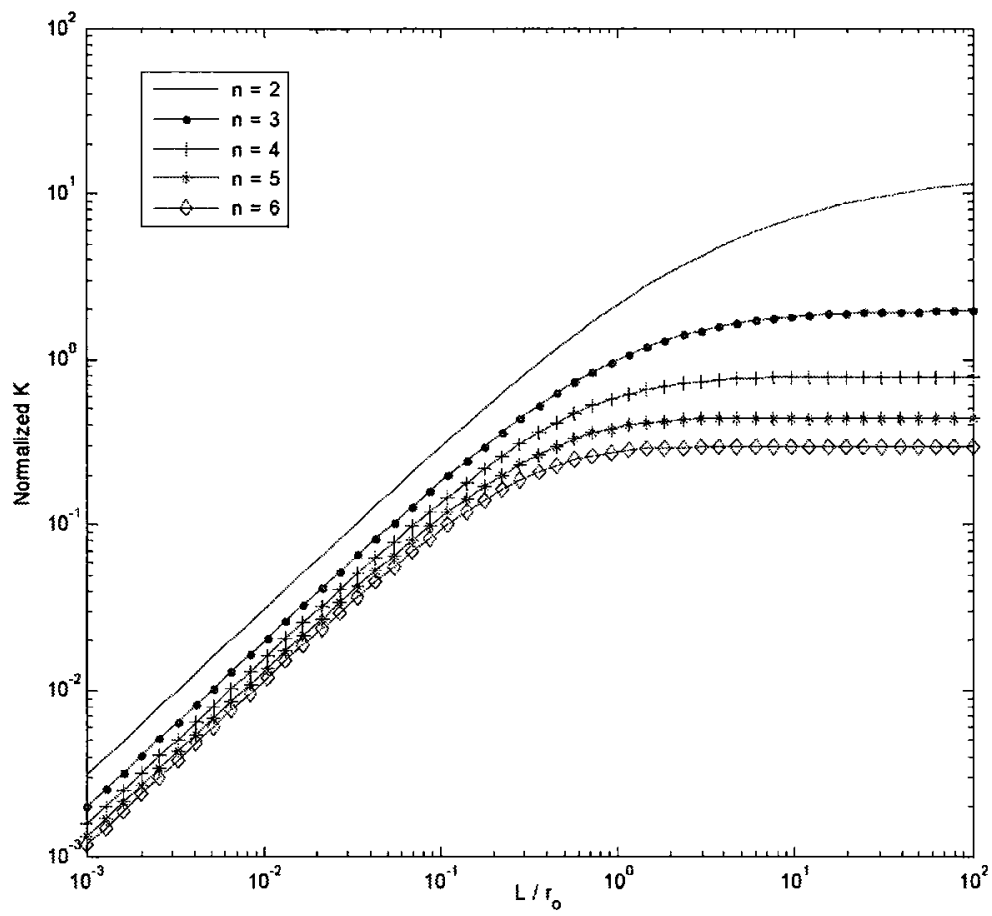

(a)

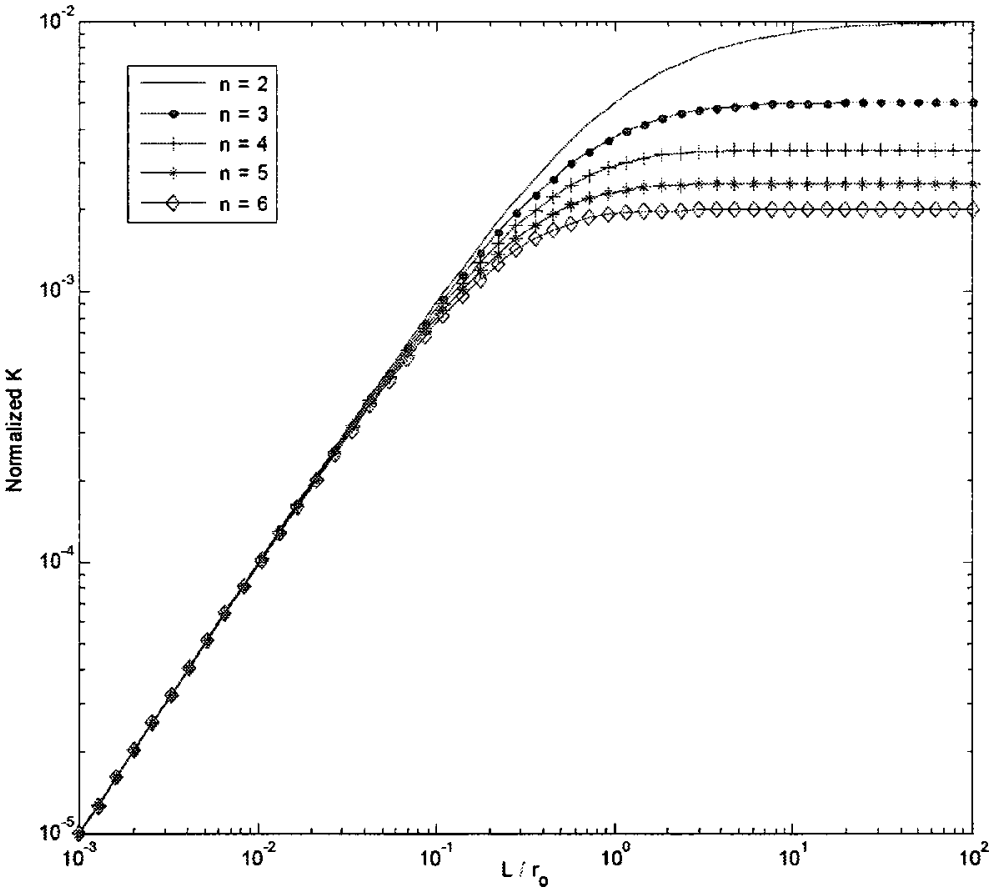

(b)

Figure 4.7: Impact of the changes in $L$ on normalized $K$, i.e, $K / \lambda r_{o}^{2}$, for Shape II. (a) $W / r_{o}=100 ;$ (b) $W / r_{o}=0.01$. 


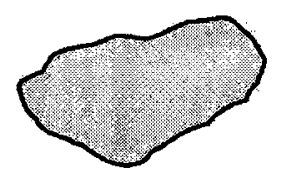

$Y$

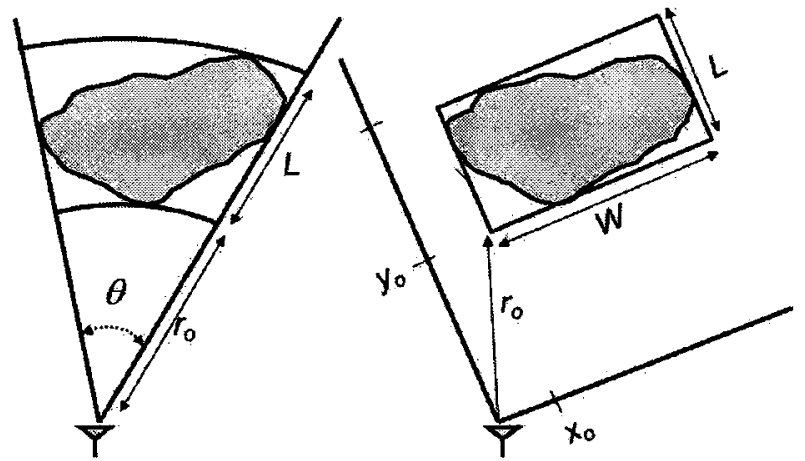

Figure 4.8: Bounding a field of an arbitrary shape by one of the regular shapes considered in this chapter.

proper constant sets an upper bound of $K$ for the worst-case scenario, i.e., when $L / r_{o}$ and $W / r_{o}$ both go to infinity. If the values of $L$ or $W$ are small compared to $r_{o}$, then the value of $\lambda r_{o}^{2}$ is scaled down by the ratio of $L / r_{o}, W / r_{o}$, or both.

\subsubsection{Upper Bounds on the Interference of a Sensor Field}

The results achieved in the previous subsections are applied here to set up upper bounds for the following:

- the average interference power of any sensor field, and

- the probability that a sensor field would generate harmful interference towards the primary user.

\subsubsection{Average Interference Power of any Sensor Field with an Arbitrary Shape}

The two special shapes analyzed above can be used to provide a conservative approximation (upper bound) for the average interference power generated by any sensor field with an arbitrary shape. 
The annular sector shape is useful in cases where the primary user has a directional antenna. In addition, it is applicable when the sensor field surrounding the primary system has the shape of a disk or ring. Generally, the approximation with a rectangular shape is more appropriate than the annular sector shape in most other cases.

The objective of the mapping is to choose the smallest shape (between Shape I and Shape II) that contains the sensor field of an arbitrary shape. Here, $r_{o}$ is the shortest distance between the shape approximating the sensor field and the primary system. An example is shown in Fig. 4.8. A tighter upper bound can also be achieved by segmenting the sensor field into subfields with an annular sector or a rectangular shape or both. The average interference power will then be equal to the sum of all average interference powers generated by the subfields.

\subsubsection{An Upper Bound on Interference Probability}

The average interference power can be used to set an upper bound for the probability that a sensor field would generate a harmful interference towards a primary user at point X. The determination of this upper bound is based on the Markov Inequality [90] which states that for a non-negative random variable $U$ which has a mean of $\mu$, and for $\alpha \geq 0$,

$$
\mathbb{P}(U \geq \alpha) \leq \frac{\mu}{\alpha} .
$$

Applying this inequality to the context of average interference power of a sensor field towards another system results in

$$
\mathbb{P}\left(I_{A} \geq I_{t h}\right) \leq \frac{\mathbb{E}\left[I_{A}\right]}{I_{t h}},
$$

where $I_{t h}$ is the threshold value for the harmful interference, and $\mathbb{E}\left[I_{A}\right]$ can be written in terms of $K$ from (4.5). 
It should be noted that the Markov inequality provides a very loose upper bound; nevertheless it can be used to deduce a conservative conclusion. For example, the primary user or the frequency regulator may require that $\mathbb{P}\left(I_{A} \geq I_{t h}\right) \leq \beta$, where $\beta$ is a limit of the interference probability. In this case, if the Markov inequality in (4.20) gives a value lower than $\beta$, then it can be deduced that the sensor field satisfies the requirement of probability of interference. Then, it is not necessary to calculate the other moments or the distribution function of the aggregated interference power generated by that sensor field. On the other hand, if (4.20) gives a value greater than $\beta$, it does not necessarily mean that the interference power generated by the senor field violated the requirement. In this case, it is required to conduct further investigations which may include calculating other moments, tighter bounds or the distribution function of the aggregate interference power.

\subsection{Upper Bound on the Interference Probability}

In this section, an upper bound on the interference probability, i.e., $P\left(I_{A} \geq I_{t h}\right)$, is established based on the knowledge of the first two moments (or cumulants) of $I_{A}$. This upper bound is used to investigate the impact of the network size on the spectrum sharing opportunities. While this upper bound could be very loose, conclusions are conservative in favor of protecting PUs. Moreover, some of the conclusions that are deduced based on this upper bound about the general behavior of the interference probability with respect to changes in the network size are valid regardless of the exact distribution of the aggregate interference power.

\subsubsection{Objective and Approach}

We are interested in studying how the interference probability and, hence, the spectrum sharing behave with respect to changes in the field size, mainly $L$, and the 
location of the field (or the exclusion region), i.e., changes in $r_{o}$. To achieve this, the distribution of $I_{A}$ or at least some of its characteristics are required. As mentioned before, the characteristic function of the aggregate interference generated by a Poisson field of interferers has been derived in $[9,12,19,20,53]$. While the characteristic function is known, the cumulative distribution function and probability density function have no closed-form expression except for a special case when the path loss exponent is 4 , the field is infinite with no exclusion region around the victim receiver [53], and the distance dependant-attenuation model is singular. Therefore, the authors in [9] and [12] end up working with approximations, and the authors in [20] use upper bounds based on Chebyshev's inequality ${ }^{1}$. Similarly, in this section, Chebyshev's inequality is utilized to establish an upper bound on the interference probability. To use this inequality, the first two moments of $I_{A}$ have to be identified. In the following sections, formulations for these moments and the upper bound on the interference probability are developed. It is worth highlighting that the expressions for the mean and the variance can be obtained from the expressions provided in Chapter 5 for the first and second cumulants, respectively. However, in this section, we follow a different approach to develop the expressions. This approach is based on conditional expectations.

\subsubsection{Mean of the Aggregate Interference}

This subsection addresses the formulation of the mean of the aggregate interference $\left(\mu_{A}\right)$ and discusses the behavior of this mean with respect to changes in the field size and location.

\footnotetext{
${ }^{1} \mathrm{~A}$ better bound for the case of the sum of independent random variables is the Chernoff upper bound [91]. This bound has a tuning parameter that can be optimized to make the bound very tight; however, it might be difficult to calculate the optimal value of this parameter. In [20], the Chernoff bound was investigated; however, the authors resort to the the Chebyshev's inequality instead of the Chernoff bound due to the computational difficulties associated with the Chernoff bound.
} 


\subsubsection{The Formulation}

Taking the expectation of $I_{A}$ in (3.1) results in

$$
\mu_{A}=\mathbb{E}\left[I_{A}\right]=\mathbb{E}\left[\sum_{i \in \Lambda} I_{i}\right]
$$

The cardinality of $\Lambda$ is assumed to be $N$. Assuming that the $I_{i}$ 's are i.i.d. random variables,

$$
\mu_{A}=\mathbb{E}[N] \mathbb{E}\left[I_{i}\right]=\mu_{N} \mu_{I}
$$

Since the number of transmitting nodes in an area follows a Poisson distribution,

$$
\mu_{N}=\lambda A
$$

where $A$ is the area of the field. Using (3.2) and the assumption that $X_{i}$ and $r_{i}$ are independent, $\mu_{I}$ can be expressed as

$$
\mu_{I}=\mu_{X} \mathbb{E}\left[r_{i}^{-n}\right]
$$

where $\mu_{X}=\mathbb{E}\left[X_{i}\right]$. In this section, we consider $r_{i} \geq r_{c}$. General expressions for the mean and variance including when $r_{i}<r_{c}$ are discussed in Chapter 5 .

Since the nodes are spatially distributed according to a PPP, the locations of the nodes follow a uniform distribution over $A$ [53]. Thus,

$$
\mathbb{E}\left[r_{i}{ }^{-n}\right]=\frac{\theta}{A(n-2)} r_{o}^{2-n}\left[1-\left(1+\frac{L}{r_{o}}\right)^{2-n}\right]
$$

From (4.22)-(4.25), $\mu_{A}$ can be obtained as

$$
\mu_{A}=\frac{\lambda \theta}{(n-2)} r_{o}^{2-n} \mu_{X}\left[1-\left(1+\frac{L}{r_{o}}\right)^{2-n}\right]
$$




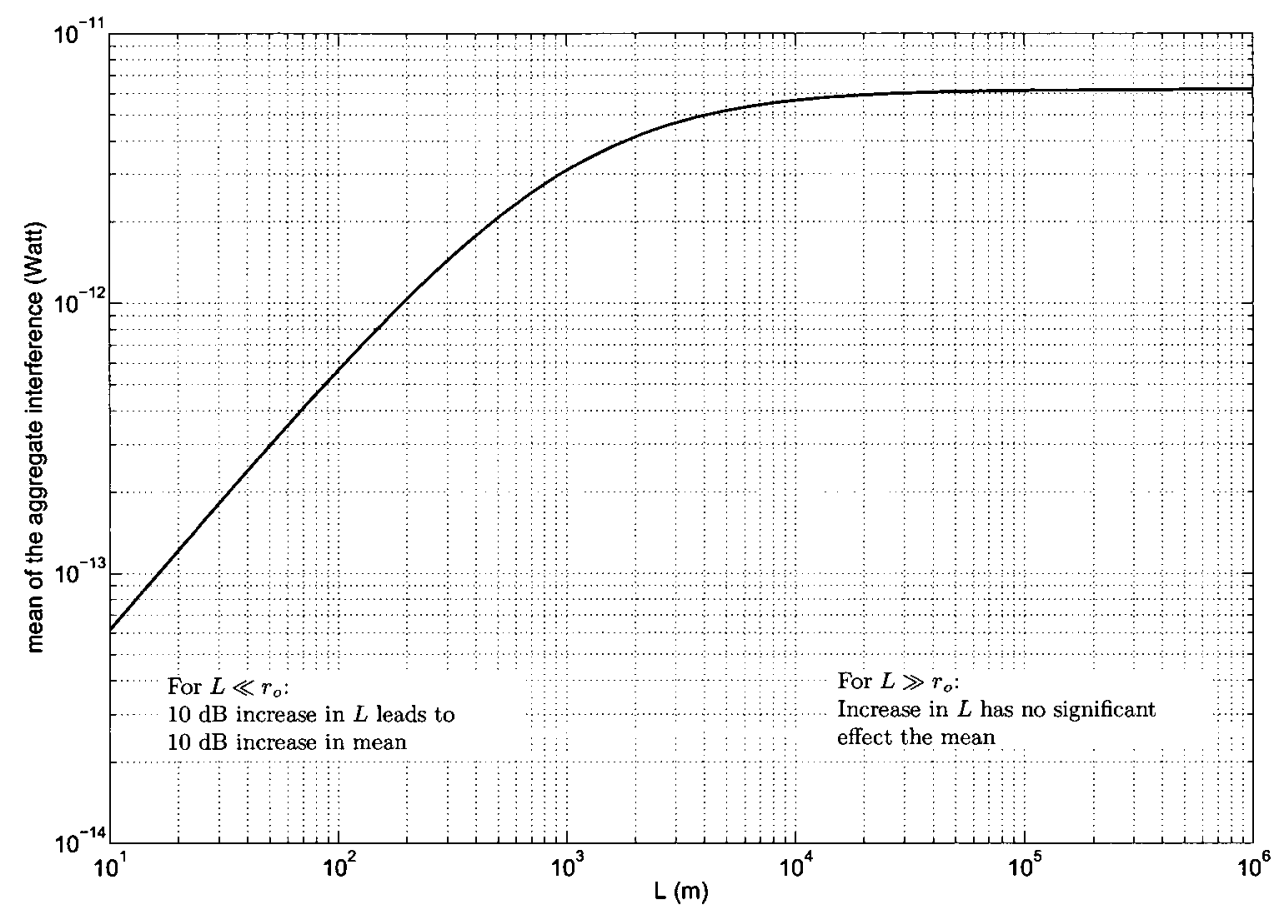

Figure 4.9: Effect of field size on the mean of the aggregate interference $\left(r_{o}=1000 \mathrm{~m}\right.$; see Table 4.1 for other assumptions used to produce this plot).

\subsubsection{Effect of Field Size and Location on $\mu_{A}$}

The behavior of the mean with respect to the changes in the field size is discussed thoroughly in Section 4.1. In brief, the mean of the aggregate interference changes linearly with changes in $L$ when $L \ll r_{o}$, see Fig. 4.9. For $L \gg r_{o}$, changes in $L$ do not significantly affect the mean of the aggregate interference, rather the mean tends to a limiting value of

$$
\mu_{A}=\frac{\lambda \theta}{(n-2)} \mu_{X} r_{o}^{2-n}
$$

This asymptotic observation agrees with the findings reported in [53] and [57] which indicate that the aggregate interference power generated by an infinite field of interferers tends to an asymptotic limit if the path loss exponent is greater than the dimensions of the field ${ }^{2}$.

\footnotetext{
${ }^{2}$ The divergence of the aggregate interference power for an infinite two-dimensional field is also discussed in $[7,55,56]$.
} 


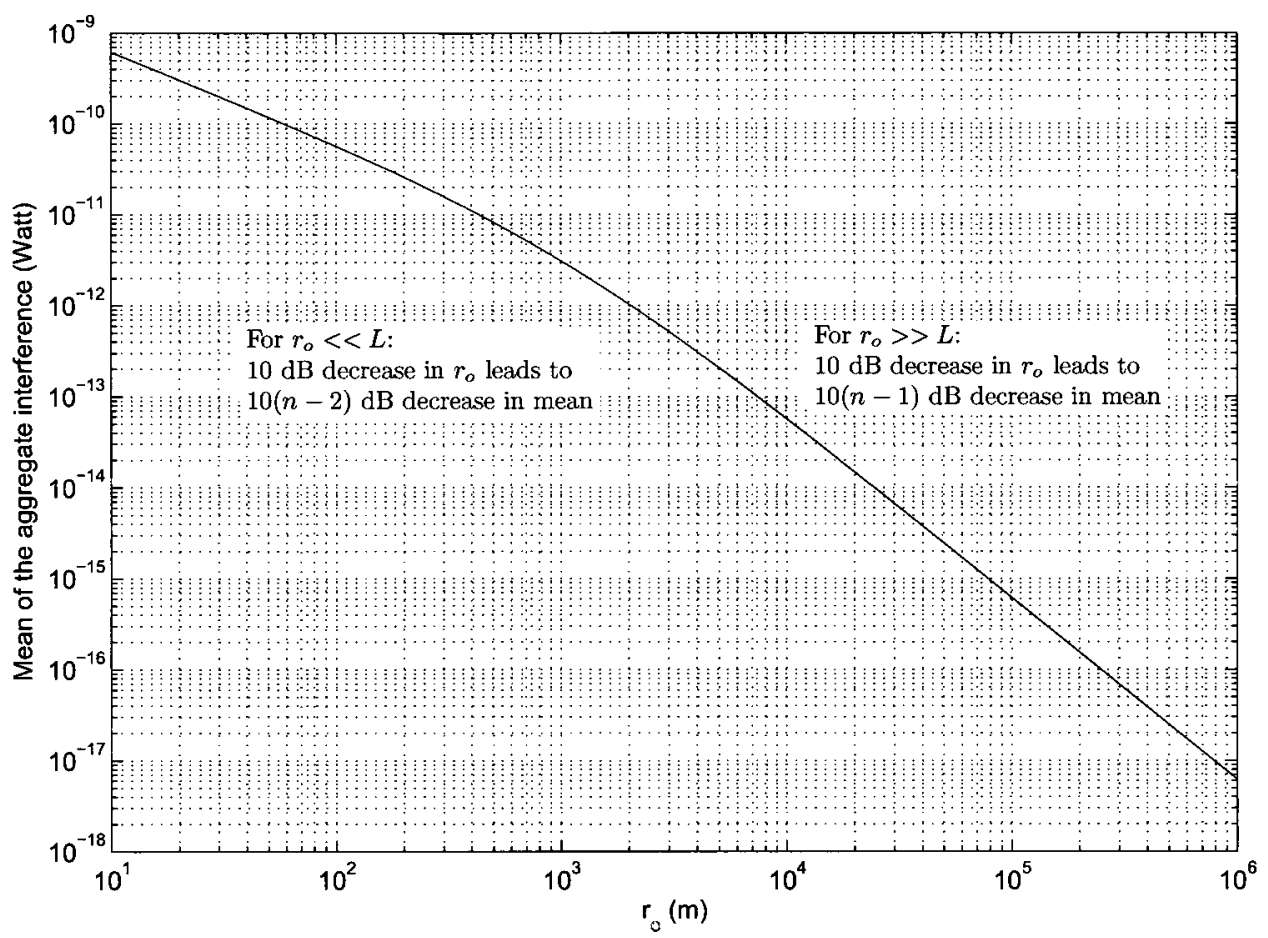

Figure 4.10: Effect of $r_{o}$ on the mean of the aggregate interference ( $L=1000 \mathrm{~m}$; see Table 4.1 for other assumptions used to produce this plot).

Table 4.1: System and Propagation Parameters Used to Produce Plots

\begin{tabular}{c|c|c}
\hline Parameter & Value & Remarks \\
\hline$n$ & 3 & \\
$P_{i}$ & $1 \mathrm{~mW}$ & \\
$G_{i}, G_{x}$ & $0 \mathrm{dBi}$ & \\
$f$ & $2.4 \mathrm{GHz}$ & \\
$d_{o}$ & $1 \mathrm{~m}$ & \\
$\theta$ & $2 \pi$ & \\
$\lambda$ & 0.01 node $/ \mathrm{m}^{2}$ & \\
$\sigma_{X}^{2}$ & $X_{i}$ assumed to be deterministic & \\
\hline
\end{tabular}

The effect of the field location on the mean of the aggregate interference is highlighted in Fig. 4.10 which can be summarized as follows: a $10 \mathrm{~dB}$ increase in $r_{o}$ leads to a $10(n-2) \mathrm{dB}$ decrease in $\mu_{A}$ for $r_{o} \ll L$; when $r_{o} \gg L$, a $10 \mathrm{~dB}$ increase in $r_{o}$ results in $10(n-1) \mathrm{dB}$ decrease in the mean of the aggregate interference. 


\subsubsection{Variance of the Aggregate Interference}

The variance of the aggregate interference of the field of secondary users is denoted by $\sigma_{A}^{2}$. This subsection presents the formulation of $\sigma_{A}^{2}$ and discusses its behavior with respect to changes in $L$ and $r_{o}$.

\subsubsection{The Formulation}

Based on the assumptions that all $I_{i}$ 's are i.i.d. random variables, it can be shown that the second moment of the aggregate interference for $N$ transmitting nodes is

$$
\mathbb{E}\left[I_{A}^{2} \mid N\right]=N\left(\sigma_{I}^{2}+\mu_{I}^{2}\right)+N(N-1) \mu_{I}^{2}
$$

Averaging (4.28) over $N$ results in

$$
\sigma_{A}^{2}=\mu_{N} \sigma_{I}^{2}+\sigma_{N}^{2} \mu_{I}^{2}
$$

Since $N$ follows a Poisson distribution,

$$
\sigma_{N}^{2}=\mu_{N}=\lambda A
$$

By noting that

$$
\begin{gathered}
\sigma_{I}^{2}=\mathbb{E}\left[X^{2}\right] \mathbb{E}\left[r_{i}^{-2 n}\right]-\mu_{I}^{2} \\
\mathbb{E}\left[r_{i}^{-2 n}\right]=\frac{\theta r_{o}^{2-2 n}}{A(2 n-2)}\left[1-\left(1+\frac{L}{r_{o}}\right)^{2-2 n}\right]
\end{gathered}
$$

and substituting them as well as (4.30) in (4.29), we obtain

$$
\sigma_{A}^{2}=\frac{\lambda \theta r_{o}^{2-2 n}}{2 n-2} \mu_{X}^{2}\left(1+\frac{\sigma_{X}^{2}}{\mu_{X}^{2}}\right)\left[1-\left(1+\frac{L}{r_{o}}\right)^{2-2 n}\right]
$$




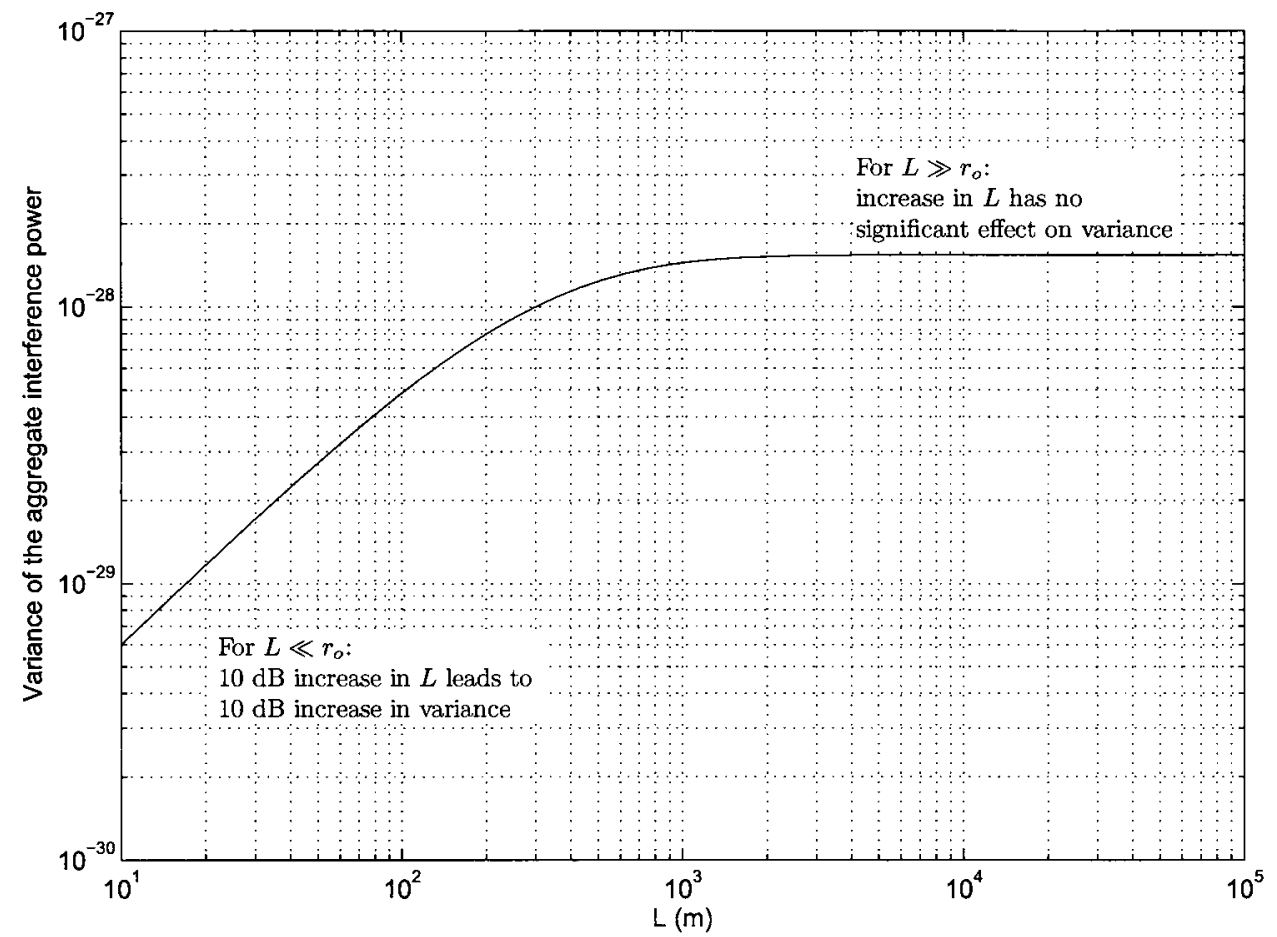

Figure 4.11: Effect of field size on variance of the aggregate interference $\left(r_{o}=1000 \mathrm{~m}\right.$; see Table 4.1 for other assumptions used to produce this plot).

which means that the randomness in $X_{i}$ causes an increase by a factor of $\left(1+\frac{\sigma_{X}^{2}}{\mu_{X}^{2}}\right)$. So, the behavior of the variance of the aggregate interference with respect to changes in the field size and location can be studied without loss of generality by assuming that $X_{i}$ is deterministic.

\subsubsection{Effect of Field Size and Location on $\sigma_{A}^{2}$}

The variance of the aggregate interference changes linearly with changes in $L$ when $L \ll r_{o}$, see Fig. 4.11. However, when $L \gg r_{o}$ changes in $L$ have no tangible effect on the variance. The variance of the aggregate interference reaches a limit with respect to the increase in $L$ to

$$
\sigma_{A}^{2}=\frac{\lambda \theta r_{o}^{2-2 n}}{2 n-2} \mu_{X}^{2}\left(1+\frac{\sigma_{X}^{2}}{\mu_{X}^{2}}\right) .
$$

Fig. 4.12 shows the effect of $r_{o}$ on the variance of the aggregate interference. A 


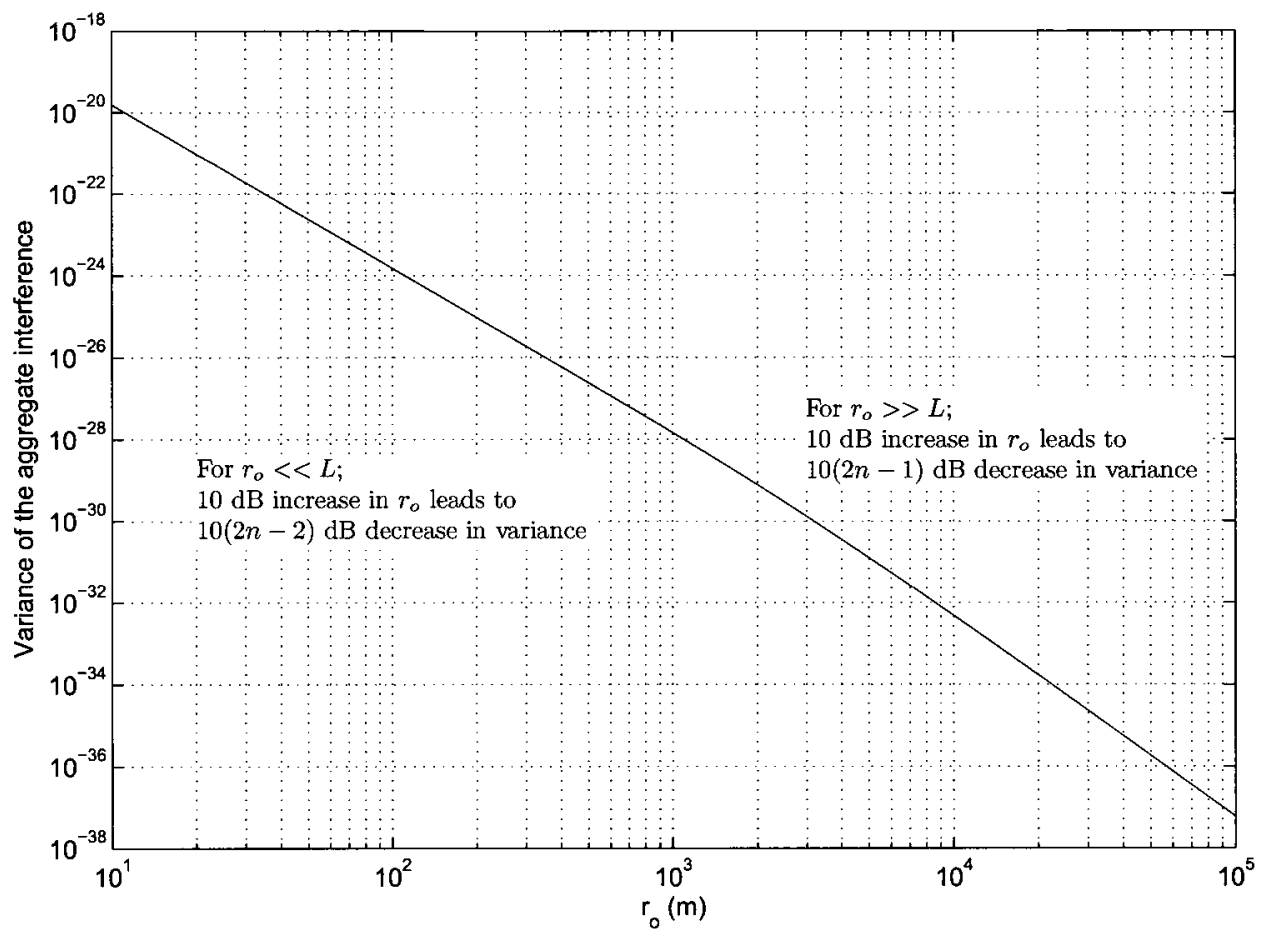

Figure 4.12: Effect of $r_{o}$ on the variance of the aggregate interference ( $L=1000 \mathrm{~m}$; see Table 4.1 for other assumptions used to produce this plot).

$10 \mathrm{~dB}$ increase in $r_{o}$ leads to $10(2 n-2) \mathrm{dB}$ decrease in the variance when $r_{o} \ll L$, and a $10(2 n-1) \mathrm{dB}$ decrease if $r_{o} \gg L$.

\subsubsection{Upper Bound on the Interference Probability}

\subsubsection{The Formulation}

An upper bound on the interference probability can be established using Chebyshev's inequality $^{3}$. This inequality states that for a random variable $X$ with mean $\mu$ and variance $\sigma^{2}[92]$

$$
P(|X-\mu| \geq t) \leq \frac{\sigma^{2}}{t^{2}}, t>0
$$

\footnotetext{
${ }^{3}$ While Chebyshev's inequality may result in loose upper bounds, the decisions deduced from these bounds are conservative and in favor of protecting the primary user.
} 
Applying this inequality to our problem results in

$$
P\left(I_{A} \geq I_{t h}\right) \leq \frac{\frac{\lambda \theta r_{o}^{2-2 n}}{2 n-2} \mu_{X}^{2}\left(1+\frac{\sigma_{X}^{2}}{\mu_{X}^{2}}\right)\left[1-\left(1+\frac{L}{r_{o}}\right)^{2-2 n}\right]}{\left(I_{t h}-\frac{\lambda \theta r_{o}^{2-n}}{(n-2)} \mu_{X}\left[1-\left(1+\frac{L}{r_{o}}\right)^{2-n}\right]\right)^{2}}
$$

provided that $I_{t h}>\mu_{A}$ where $I_{t h}$ could be the maximum level of the tolerable interference by the primary user.

The expression (4.36) can be used to understand the effect of the randomness in $X_{i}$ on the probability of interference. As mentioned before, the randomness in $X_{i}$ may be due to the fluctuations in the transmit power, multipath fading, and more importantly, the shadowing effect. The randomness in $X_{i}$ shifts up the upper bound

of the interference probability by $10 \log \left(1+\frac{\sigma_{X}^{2}}{\mu_{X}^{2}}\right) \mathrm{dB}$. So, without loss of generality, the behavior of the upper bound on the interference probability with respect to changes in field size and location can be studied with the assumption that $X_{i}$ is deterministic. The results could be adjusted with a proper scaling factor (or a proper dB shift) to reflect the randomness in $X_{i}$.

\subsubsection{Effect of Field Size and Location on the Interference Probability}

Before discussing the effect of changes in the field size and location on the interference probability, it is important to highlight the following: the Chebyshev inequality is valid when $I_{t h}>\mu_{A}$; however, $\mu_{A}$ would change with changes in the field size and location. Therefore, in our plots we make sure that $I_{t h}>\mu_{A}$ over the ranges of $L$ or $r_{o}$ considered in the plots. In practice, the value of $I_{t h}$ is determined by the regulator or the primary user, as such, the secondary user has no control over it.

The behavior of the upper bound on the interference probability with respect to changes in the field size is shown in Fig. 4.13. The upper bound on the interference probability increases linearly with the increase in $L$ for $L \ll r_{o}$. If $L \gg r_{o}$, then the 


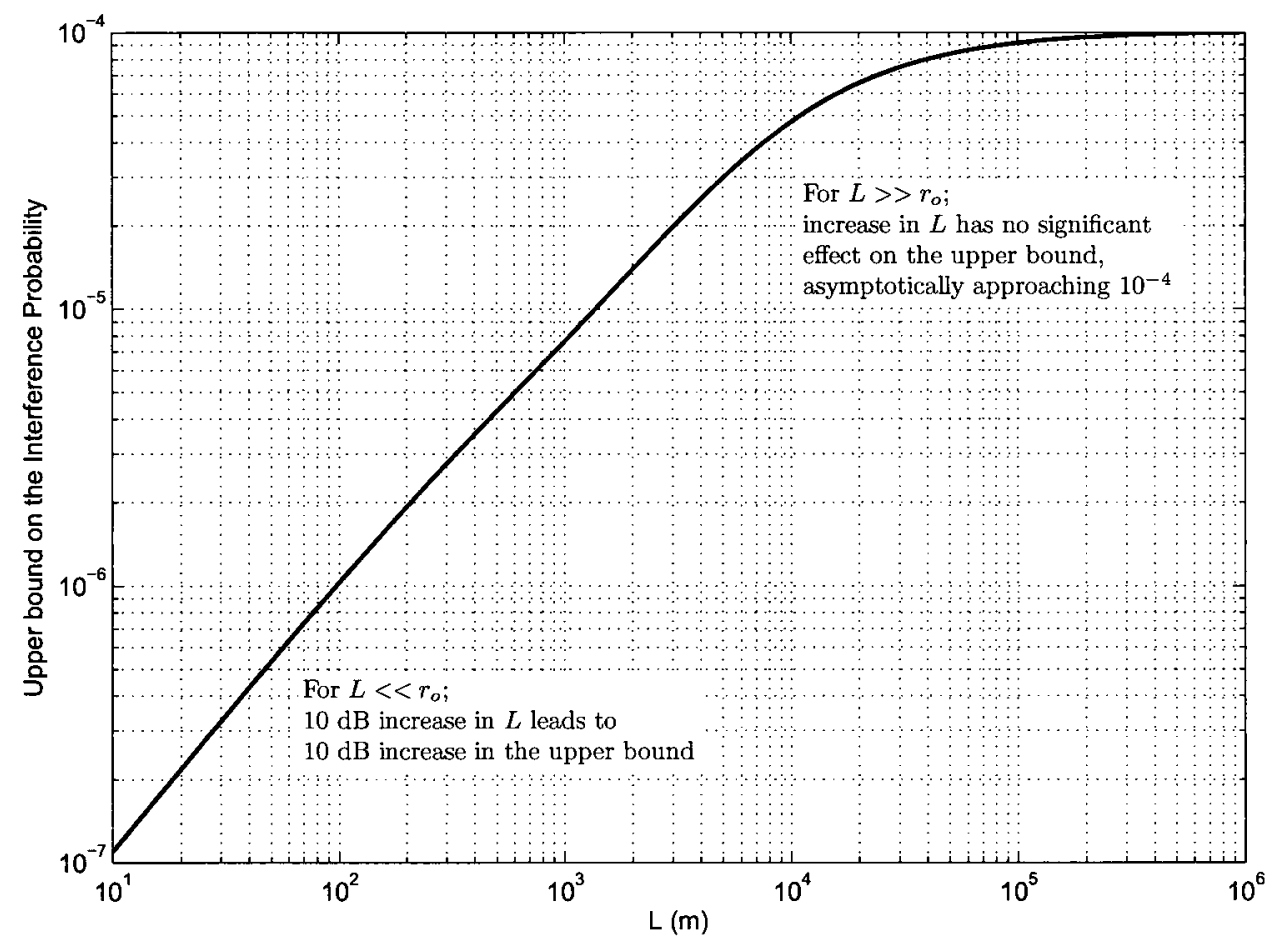

Figure 4.13: Effect of field size on interference probability $\left(r_{o}=1000 \mathrm{~m}\right.$; see Table 4.1 for other assumptions used to produce this plot).

increase in $L$ has no or negligible effect on the upper bound. In this case, the upper bound saturates at

$$
P\left(I_{A} \geq I_{t h}\right) \leq \frac{\frac{\lambda \theta}{2 n-2} r_{o}^{2-2 n} \mu_{X}^{2}\left(1+\frac{\sigma_{X}^{2}}{\mu_{X}^{2}}\right)}{\left(I_{t h}-\frac{\lambda \theta}{n-2} r_{o}^{2-n} \mu_{X}\right)^{2}}
$$

Fig. 4.14 shows the behavior of the upper bound on the interference probability with respect to changes in $r_{o}$. A $10 \mathrm{~dB}$ increase in $r_{o}$ leads to a $10(2 n-2) \mathrm{dB}$ decrease in the upper bound if $r_{o} \ll L$; when $r_{o} \gg L$, a $10 \mathrm{~dB}$ increase in $r_{o}$ leads to a $10(2 n-1)$ $\mathrm{dB}$ decrease in the upper bound. Table 4.2 summarizes some asymptotic expressions, i.e., when $L \gg r_{o}$ and $L \ll r_{o}$, for the probability upper bound, variance, and mean of the aggregate interference. 


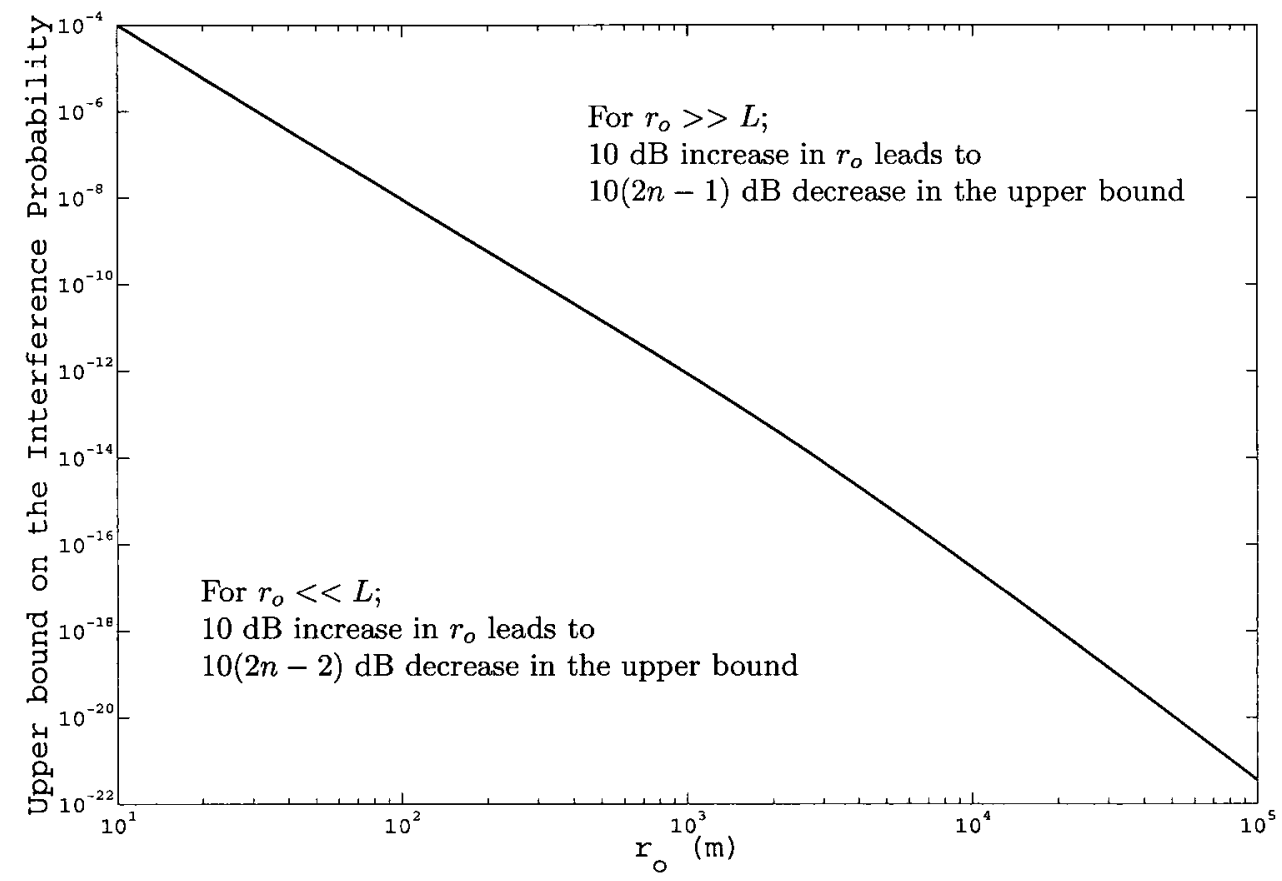

Figure 4.14: Effect of field location on interference probability ( $L=1000 \mathrm{~m}$; see Table 4.1 for other assumptions used to produce this plot).

Table 4.2: Summary of Asymptotic Expressions

\begin{tabular}{l|c|c}
\hline & For $L \gg r_{o}$ & For $L \ll r_{o}$ \\
\hline$P\left(I_{A} \geq I_{t h}\right) \leq$ & $\frac{\frac{1}{2 n-2} \lambda \theta r_{o}^{2-2 n} \mu_{X}^{2}\left(1+\frac{\sigma_{X}^{2}}{\mu_{X}^{2}}\right)}{\left(I_{t h}-\frac{1}{n-2} \lambda \theta r_{o}^{2-n} \mu_{X}\right)^{2}}$ & $\frac{\lambda \theta r_{o}^{1-2 n} L \mu_{X}^{2}\left(1+\frac{\sigma_{X}^{2}}{\mu_{X}^{2}}\right)}{\left(I_{t h}-\lambda \theta r_{o}{ }^{1-n} L \mu_{X}\right)^{2}}$ \\
\hline$\sigma_{A}^{2}=$ & $\frac{1}{2 n-2} \lambda \theta r_{o}^{2-2 n} \mu_{X}^{2}\left(1+\frac{\sigma_{X}^{2}}{\mu_{X}^{2}}\right)$ & $\lambda \theta r_{o}^{1-2 n} L \mu_{X}^{2}\left(1+\frac{\sigma_{X}^{2}}{\mu_{X}^{2}}\right)$ \\
\hline$\mu_{A}=$ & $\frac{1}{n-2} \lambda \theta r_{o}^{2-n} \mu_{X}$ & $\lambda \theta r_{o}{ }^{1-n} L \mu_{X}$ \\
\hline
\end{tabular}

\subsubsection{Effect of Field Size and Location on Spectrum Sharing Opportuni- ties}

We consider a spectrum sharing opportunity exists if the condition in (3.6) is satisfied. Therefore, for a certain value of $I_{t h}$, the upper bound obtained in (4.36) will be compared to (3.6). If the upper bound is less than or equal to $\beta$, then the aggregate interference of the secondary network is considered to be non-harmful to the primary user (non-interfering region). Otherwise, the secondary network may cause harmful 
interference to the primary user (interfering region).

If the secondary network operates in an interfering region, then it may use the spectrum of the primary user during the absence of the primary user. However, when the primary user starts using the spectrum, then the secondary user has to evacuate the spectrum or to adapt its transmission parameters to move to the non-interfering region. Therefore, the secondary user has to have a technology such as "cognitive radio" to be able to sense the presence of the primary user, and to perform a proper action accordingly $[5,6]$.

On the other hand, if the secondary network operates in the non-interfering region, then it can concurrently and continuously share the spectrum with the primary user without the need to implement any spectrum sensing technique or cognition related function. This result could be used towards solving the spectrum problem facing wireless sensor networks (WSN)s without increasing the complexity of the sensor nodes. Currently, Wireless Sensor Networks (WSNs) are operating in unlicensed bands, e.g., 2.4 GHz. These bands are becoming over-crowded which may severely affect the operation of the WSNs. Alternatively, the WSNs could share a licensed band with a primary user. However, this sharing usually requires spectrum sensing and cognitive radio functionalities. Adding these functionalities may increase the complexity of the sensor nodes to a level that is economically infeasible with today's technologies especially for massive deployments [88]. It has been shown in this section that under certain conditions secondary users (WSNs in this example) could concurrently and continuously share the spectrum with a primary user without the need to have any spectrum sensing techniques or cognitive radio functionalities.

The effect of the field size and location on determining the interfering and noninterfering regions and on the spectrum sharing opportunities are discussed in the following subsections. Without loss of generality, the numerical examples and results 


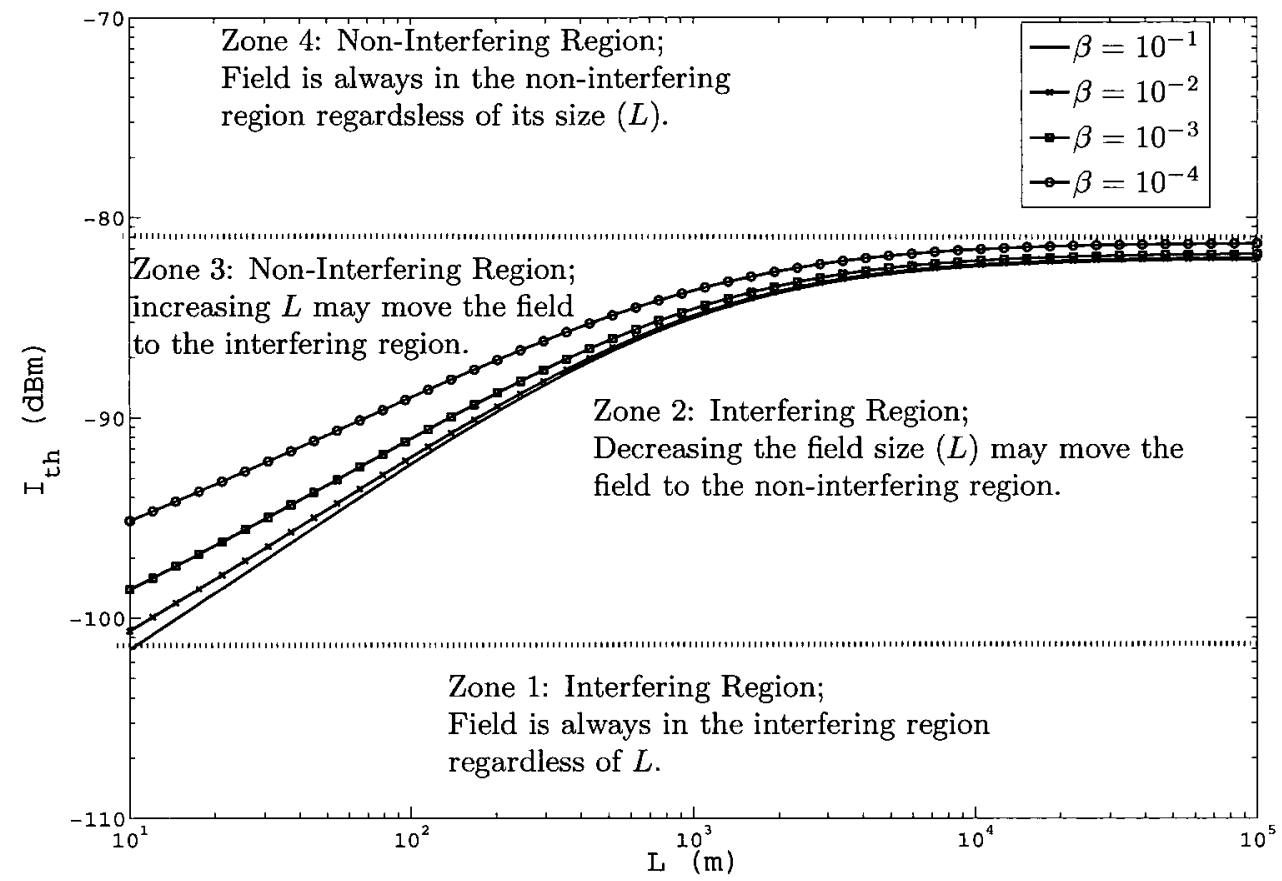

Figure 4.15: Field size and spectrum sharing opportunities $\left(r_{o}=1000 \mathrm{~m}\right.$; see Table 4.1 for other assumptions used to produce this plot).

shown later in this section are based on the following model for $X_{i}$ :

$$
X_{i}=P_{i} G_{i} G_{x}\left(\frac{\gamma}{4 \pi}\right)^{2} d_{o}^{n-2} h_{i} 10^{\frac{S_{i}}{10}}
$$

where $P_{i}$ is the transmitted power by node $i, G_{i}$ is its antenna gain, $G_{x}$ is the antenna gain of the primary user, $\gamma$ is the wavelength of the carrier frequency, $d_{o}$ is the close-in reference distance, $h_{i}$ reflects the effect of the multipath fading on the received power, and $S_{i}$ is a Gaussian random variable modeling the shadowing effect.

\subsubsection{Field Size and Spectrum Sharing Opportunities}

The discussion in this subsection is based on Fig. 4.15. This figure has four curves; each curve corresponds to a certain value of $\beta$. Each one of these curves divides the figure into two main regions: interfering (comprised of zones 1 and 2) and noninterfering (comprised of zones 3 and 4) regions. 
If the maximum interference probability that a primary user can tolerate is $\beta$ (e.g., 0.01 ), then the aggregate interference generated by the secondary network is not considered to be harmful if the interference probability is less than $\beta$. Therefore, the region in Fig. 4.15 above the curve of $\beta=0.01$ is considered to be a non-interfering region. In this case, a secondary network could concurrently and continuously share the spectrum with the primary user without the need to have spectrum sensing techniques or cognitive radio capabilities.

The non-interfering region in Fig. 4.15 can be divided into two zones: zone 3 and zone 4 . In zone 4 , the secondary network does not cause harmful interference towards the primary users regardless of the field size, $L$. Therefore, expanding the field does not eliminate any spectrum sharing opportunity. In zone 3 , on the other hand, the increase in $L$ may move the secondary network from the non-interfering region to the interfering region eliminating a spectrum sharing opportunity.

For a specific $\beta$ value, the part of Fig. 4.15 below the curve corresponding to that $\beta$ (e.g., 0.01) is considered to be an interfering region because the interference probability in this part is grater than 0.01 . The interfering region can also be divided into two zones. In the first one (zone 1), a reduction in $L$ never moves the secondary network from the interfering region to the non-interfering region except when there is no transmitting node. Therefore, controlling the field size here does not create a spectrum sharing opportunity. In the other zone (zone 2), on the other hand, a reduction in $L$ may lead to moving the secondary network from the interfering region to the non-interfering region which creates a spectrum sharing opportunity.

At this stage we can comment on the impact of the field size on the spectrum sharing opportunities. It has been shown that an increase in the field size may eliminate spectrum sharing opportunities. However, there are some cases where the spectrum sharing opportunities are not affected by the increase in the field size even if the field size grows to infinity. Moreover, it has been demonstrated that the reduction 


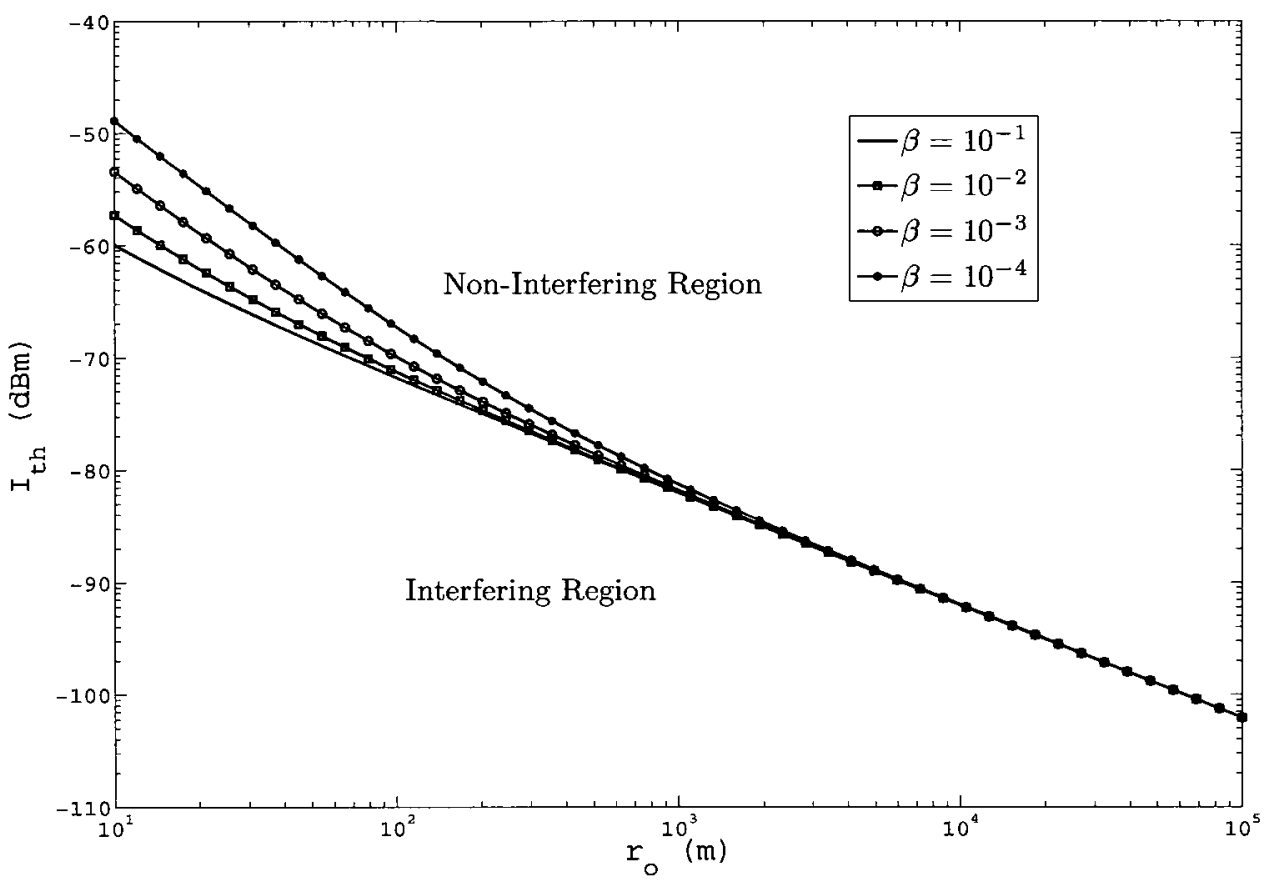

Figure 4.16: Field location and spectrum sharing opportunities $(L \rightarrow \infty$; see Table 4.1 for other assumptions used to produce this plot).

in the field size may open spectrum sharing opportunities. On the other hand, there are some other cases where the reduction in the field size does not open any spectrum sharing opportunities.

\subsubsection{Field Location and Spectrum Sharing Opportunities}

Fig. 4.16 has four curves. Each curve corresponds to a certain value of $\beta$. Each one of these curves divides the figure into two regions: interfering and non-interfering regions.

A parameter that the secondary network may use to move from an interfering region to a non-interfering region is $r_{o}$. By knowing the maximum acceptable interference probability $\beta$ in (3.6) and $I_{t h}$, Fig. 4.16 can be used to find the minimum exclusion region $\left(r_{o}\right)$ satisfying these requirements. For example, if $I_{t h}=-80 \mathrm{dBm}$, then $r_{o}$ should be greater than or equal to $630 \mathrm{~m}$ to ensure that the interference 
probability is less than or equal to 0.1 .

Fig. 4.16 also reveals an interesting situation. The right side of the plot where the lines of different $\beta$ 's overlap may suggest that for sufficiently high $r_{o}$ there is no need to know the distribution of the aggregate interference; knowing only the mean is sufficient. In other words, the aggregate interference in this case is tending towards a deterministic quantity. Similar observations are made in [19] while discussing the ratio of the standard deviation of the aggregate interference to its mean.

\subsection{Conclusions}

In this chapter, we provide an asymptotic analysis of the average interference power generated by a WSN towards a primary user sharing the same frequency band. Applying this analysis to two special but important wireless sensor field shapes produces closed-form expressions. These expressions provide further insight into the behavior of the average interference power generated by a sensor field towards a primary user. based on exponential power decay with distance. Moreover, the analysis and results presented in this chapter can also be applied to the case when a WSN shares an unlicensed frequency band with another set of unlicensed users.

Results reported in this chapter reveal that the average interference power does not depend on the exact values of the depth or width of the sensor field, but depends on their ratio to the shortest distance between the field and the primary user. If this ratio is much smaller than 1, then the average interference power changes linearly with changes in this ratio. On the other hand, if the ratio is much greater than 1 , then the average interference power is upper bounded by a constant regardless of how big the field is. An exception to this is when the path loss exponent is 2. In this case, the average interface power changes logarithmically with changes in the field size.

It has been demonstrated that the annular sector shape or rectangular shape 
presented in this chapter can be used to provide a conservative approximation (an upper bound) for the average interference power of a sensor field with any arbitrary shape.

This chapter also studies the impact of the field size of secondary users on spectrum sharing opportunities. The study shows that an increase in the field size may eliminate the spectrum sharing opportunities. However, there are some cases where the spectrum sharing opportunities are not reduced by the increase in the field size even when the field size grows to infinity.

The results in this chapter show the possibility of a secondary network to concurrently and continuously share the spectrum with a primary network without the need for spectrum sensing techniques or other cognitive radio functionalities. This observation hints at a promising spectrum sharing solution for the wireless sensor networks which are facing interference challenges in the crowded unlicensed spectrum. 


\section{Chapter 5}

\section{Cumulants-Based Characterization of the Aggregate Interference Power and Spectrum Sharing}

A cumulant-based characterization of the aggregate interference is an attractive approach. A number of recent papers in the literature have dealt with cumulants of the aggregate interference but under specific scenarios. In this chapter, we introduce a simple yet comprehensive method to determine the cumulants of the aggregate interference power originating from a wireless network. This method is quite general and applicable for finite and infinite network sizes, and it is flexible to encompass different system and propagation parameters such as large-scale fading, small-scale fading or even composite fading. Moreover, this chapter revisits the impact of the spatial size of the secondary network on spectrum sharing opportunities, which is discussed preliminarily in Chapter 4. The study in this chapter is based on an approximation of the distribution of the aggregate interference power and the interference probability, rather than an upper bound. 


\subsection{Introduction}

While the moments and cumulants are closely related, cumulants have some properties making them more attractive for characterizing the aggregate interference of a Poisson field of interferers. Among these properties is that the $m$ th cumulant of a sum of independent random variables is equal to the sum of the individual $m$ th cumulants [77]. Combining this property with the independence property of disjoint Poisson fields provides a powerful tool in characterizing the aggregate interference in a wireless network.

To the best of our knowledge, few papers in the literature have dealt with cumulants of the aggregate interference power. These papers focus on specific scenarios. For example, [7] and [8] only consider the first cumulant, i.e., the mean. The authors in [9] and [10] deal with cumulants for non-fading scenarios, [11] provides an integral form to compute the cumulants for out-of-cell interference in CDMA networks,

and [12] considers an infinite field with an exclusion region. Extending these results and generalizing them for a wide range of scenarios are among the contributions of this thesis.

In this chapter, we characterize the distribution of the aggregate interference using cumulants. We provide a very simple yet powerful method to determine the cumulants. The method is flexible enough to be applicable to a wide range of scenarios including, but not limited to, the following: finite fields, infinite fields, different small-scale and large-scale fading distributions (e.g., Rayleigh, Rician, lognormal, generalized- $K$ ), and variations in power levels.

\subsection{Cumulants of $I_{A}$}

One of the contributions of this thesis is stated in the following proposition. To interpret this proposition, it is helpful to imagine that the field of interferers would 
virtually collapse to a subfield with an effective area $A_{\text {eff }}$, an inner radius $r_{o}$, and an outer radius $r_{\text {eff }}$. The average number of interfering nodes within this subfield is $N_{\text {eff }}$.

Proposition 1. The mth cumulant of the distribution of the aggregate interference power received by a victim receiver at the origin from an annulus-shaped Poisson field of i.i.d. interferers is

$$
\kappa_{m}\left(I_{A}\right)=N_{\mathrm{eff}}(m) \tilde{\mu}_{m}\left(I_{r_{o}}\right)
$$

where $N_{\mathrm{eff}}(m)$ is the average number of interfering nodes within a radius of $r_{\mathrm{eff}}(m)$ from the victim receiver, and $\tilde{\mu}_{m}\left(I_{r_{o}}\right)$ is the mth raw moment of the distribution of the interference power received by the victim receiver from an interfering node at distance $r_{0}$. We then have

$$
N_{\mathrm{eff}}(m)=\lambda A_{\mathrm{eff}}(m)
$$

where

$$
A_{\mathrm{eff}}(m)=\frac{1}{2} \theta\left[r_{\mathrm{eff}}^{2}(m)-r_{o}^{2}\right]
$$

and

$$
\begin{aligned}
r_{\mathrm{eff}}(m) & =\hat{r} \sqrt{1+\frac{2}{m n-2}\left(1-\left[\frac{\hat{r}}{r_{o}+L}\right]^{m n-2}\right)}, \\
\hat{r} & =\max \left(\min \left(r_{c}, r_{o}+L\right), r_{o}\right)
\end{aligned}
$$

Note that $\tilde{\mu}_{m}\left(I_{r_{o}}\right)=\tilde{\mu}_{m}(X)\left[g\left(r_{o}\right)\right]^{m}$ where $\tilde{\mu}_{m}(X)$ is the mth raw moment of $X_{i}$.

Proof. Using Campbell's theorem for a Poisson point process $[1,14]$, it can be shown that

$$
\phi_{I_{A}}(\omega)=\exp \left(-\theta \lambda \int_{r_{0}}^{r_{o}+L} \int_{0}^{\infty}\left[1-e^{j \omega x g(r)}\right] f_{X}(x) r d x d r\right)
$$

where $\phi_{I_{A}}(\omega)$ is the characteristic function of $I_{A}, f_{X}(x)$ is the PDF of $X_{i}$, and $j=$ 
$\sqrt{-1}$. Denoting the characteristic function of $X_{i}$ by $\phi_{X}(\omega),(5.5)$ can be rewritten as

$$
\phi_{I_{A}}(\omega)=\exp \left(-\theta \lambda \int_{r_{o}}^{r_{o}+L}\left[1-\phi_{X}(\omega g(r))\right] r d r\right) .
$$

Using (5.6) and (2.1),

$$
\kappa_{m}\left(I_{A}\right)=\theta \lambda \tilde{\mu}_{m}(X) \int_{r_{o}}^{r_{o}+L} g^{m}(r) r d r .
$$

Then, the proof follows directly from (5.7) with some mathematical manipulations and rearrangements.

\section{Remarks:}

- Equation (5.1) is simple yet flexible in the sense that it can be applied to a wide range of scenarios such as finite fields, infinite fields (see Chapter 5.6), and to different fading distributions (see Section 5.3).

- Moreover, (5.1) is applicable for many field's topologies, including when the PU-RX is in the middle of a secondary network or away from it.

- Equations (5.2), (5.3), and (5.4) can be combined to express $\kappa_{m}$ in an expanded 
form as

$$
\kappa_{m}\left(I_{A}\right)=\left\{\begin{array}{cl}
\frac{1}{n m-2} \lambda \theta \tilde{\mu}_{m}(X) r_{o}^{2-m n}\left[1-\left(\frac{r_{o}}{r_{o}+L}\right)^{m n-2}\right], & 0<r_{c} \leq r_{o} \leq r_{o}+L \\
\frac{1}{2} \lambda \theta \tilde{\mu}_{m}(X) r_{c}^{-m n}\left[r_{c}^{2}-r_{o}^{2}\right]+ & \\
\frac{1}{n m-2} \lambda \theta \tilde{\mu}_{m}(X) r_{c}^{2-m n}\left[1-\left(\frac{r_{c}}{r_{o}+L}\right)^{m n-2}\right], & 0 \leq r_{o}<r_{c} \leq r_{o}+L . \\
\frac{1}{2} \lambda \theta \tilde{\mu}_{m}(X) r_{c}^{-m n}\left[\left(r_{o}+L\right)^{2}-r_{o}^{2}\right], & 0 \leq r_{o} \leq r_{o}+L<r_{c}
\end{array}\right.
$$

\subsection{Effect of the Distribution of $X$ on Cumulants}

As indicated in Chapter 3, the random variable $X_{i}$ encompasses many system and channel parameters. It might be modeled as the multiplication of some deterministic and random variables reflecting the effect of different parameters, such as fluctuations in power level and antenna gains, multipath fading, and shadow fading. From (5.1) and $\tilde{\mu}_{m}\left(I_{r_{o}}\right)=\tilde{\mu}_{m}(X)\left[g\left(r_{o}\right)\right]^{m}$, it is clear that the distribution of $X_{i}$ has a major influence on $\kappa_{m}$, and hence the distribution of the aggregate interference power. A similar observation on the influence of the fading distribution, equivalently of the distribution of $X_{i}$, on the aggregate interference appears in [16].

Following are some examples of $\kappa_{m}$ under different distributions of $X_{i}$. While $X_{i}$ in our model is more general than just fading, examples given here consider fading distributions only. 


\subsubsection{Multipath Fading}

A general model that could be used to reflect the multipath fading on the interference power is the Gamma distribution (under the assumption of Nakagami fading for the interference signal amplitude). The PDF of the Gamma distribution is

$$
f_{X}(x)=\left(\frac{\nu}{\Omega}\right)^{\nu} \frac{x^{\nu-1}}{\Gamma(\nu)} e^{-\frac{\nu}{\Omega} x}, x>0, \nu \geq \frac{1}{2},
$$

where $\nu$ is the shape parameter, $\Gamma(\cdot)$ is the Gamma function, and $\Omega$ is the average power, i.e., $\mathbb{E}[X]$, which is commonly assumed to be equal to unity [93]. The $m$ th raw moment of the Gamma distribution with $\Omega=1$ can be expressed as

$$
\tilde{\mu}_{m}(X)=\nu^{-m} \frac{\Gamma(\nu+m)}{\Gamma(\nu)} .
$$

Therefore,

$$
\kappa_{m}\left(I_{A}\right)=N_{\mathrm{eff}}(m)\left[g\left(r_{o}\right)\right]^{m} \nu^{-m} \frac{\Gamma(\nu+m)}{\Gamma(\nu)} .
$$

The exponential distribution (corresponding to Rayleigh fading for the interference signal amplitude) is a special case of the Gamma distribution, when $\nu=1$.

\subsubsection{Shadow Fading}

Shadow fading is usually modeled by a lognormal random variable whose mean and standard deviation in the $\mathrm{dB}$ domain are zero and $\sigma_{\mathrm{dB}}$, respectively. Let $X_{i}=10^{S_{i} / 10}$, representing the shadowing effect in the linear domain, where $S_{i} \sim \mathcal{N}\left(0, \sigma_{\mathrm{dB}}^{2}\right)$. It can be shown that

$$
\tilde{\mu}_{m}(X)=e^{\frac{1}{2}\left(m \frac{\ln 10}{10} \sigma_{\mathrm{dB}}\right)^{2}}
$$

and 


$$
\kappa_{m}\left(I_{A}\right)=N_{\mathrm{eff}}(m)\left[g\left(r_{o}\right)\right]^{m} e^{\frac{1}{2}\left(m \frac{\ln 10}{10} \sigma_{\mathrm{dB}}\right)^{2}}
$$

after a proper normalization of constant terms.

\subsubsection{Composite Fading}

If the wireless channel suffers from multipath and shadow fading simultaneously, $X_{i}$ can be modeled as the product of two independent random variables $[14,82]$. Assuming these random variables are Gamma (for multipath fading) and lognormal (for shadow fading), the cumulants of the normalized aggregate interference can be expressed as

$$
\kappa_{m}\left(I_{A}\right)=N_{\mathrm{eff}}(m)\left[g\left(r_{o}\right)\right]^{m} e^{\frac{1}{2}\left(m^{\frac{\ln 10}{10}} \sigma_{\mathrm{dB}}\right)^{2}} \nu^{-m} \frac{\Gamma(\nu+m)}{\Gamma(\nu)}
$$

\subsection{Generalizations of the Cumulant-Based Characterization}

The formulations of the cumulants and the discussions on spectrum sharing opportunities can be generalized for other cases including the following situations.

\subsubsection{A Field with an Irregular Shape}

If the PU-RX is inside a field of SU-TXs but the shape of this field is not a regular disk or annular sector shape, then the results obtained before are applicable to this case provided that the distance between the PU-RX and the nearest edge of the field is much greater than the radius of the exclusion region around the PU-RX. Otherwise, the field can be segmented into disjoint segments and the total $m$ th cumulant will be the sum of $m$ th cumulants of each segment, i.e.,

$$
\kappa_{m}=\sum_{i} \kappa_{m_{i}}
$$


where $\kappa_{m_{i}}$ is the $m$ th cumulant of segment $i$. This result is obtained from the independence property of disjoint Poisson fields and the property of the cumulants of independent random variables that states the $m$ th cumulant of the sum of independent random variables is the sum of their individual $m$ th cumulants.

\subsubsection{A Field of Heterogeneous Networks}

The results obtained before have been based on the assumption that the $X_{i}$ are identically distributed. However, for a heterogeneous network (which can be visualized

as the overlap of different types of networks) the distributions of the $X_{i}$ might be non-identical. If each network by itself makes a Poisson field and those sub-networks are independent of each other, then it can be shown that the $m$ th cumulant of the heterogeneous network is the sum of the $m$ th cumulant of each of the individual networks. It is worth noting that a similar approach, i.e., considering a heterogeneous network as a set of overlapping homogeneous networks, is used in [63] to develop a moment generating function of the aggregate interference in heterogeneous networks.

\subsubsection{Many Primary Users}

While the system model considers a single PU-RX, results of this chapter are still useful for cases having many PU-RXs (composing a primary network). For example, if a primary network allows spectrum sharing only if none of its PU-RXs is experiencing harmful interference, then analysis in this chapter should be applied to the PU-RX representing the worst case scenario. An example of a PU-RX representing the worst case scenario is when this PU-RX is at the center of the secondary network given that a portion of the primary network overlaps with the center of the secondary network. Another example is when the primary network and secondary network partially overlap at the edges. In this case, the deepest PU-RX within the secondary network will be considered for the analysis. A third example is when the primary 
network and secondary network do not overlap. Then, the analysis will be based on the closest PU-RX to the secondary network. Furthermore, the results in this chapter could be used to establish an upper-bound of the interference probability, or a lower bound of spectrum sharing opportunities. Generally, the results of this chapter can be extended to investigate the spectrum sharing opportunities that a secondary network may have in the presence of a primary network that imposes a network level harmful interference metric, which could be a generalization of the user level harmful interference metric shown in (3.6).

\subsection{Cumulants-based Approximations of the Distribution of the Aggregate Interference Power}

The distribution of $I_{A}$ can be approximated using a finite set of its cumulants. This section discusses some of these cumulant-based approximations.

\subsubsection{Edgeworth Series Expansion}

The Edgeworth expansion is a simple approach used to expand the logarithm of the characteristic function using its Taylor series expansion. Based on this expansion and knowing the cumulants of a random variable, the PDF of this random variable can be approximated [11]. A detailed discussion of the Edgeworth expansion for approximating a PDF is provided in [78].

The Edgeworth series expansion is used in $[9,11,12]$ to approximate the PDF of the aggregate interference. An approximation of the PDF of $I_{A}$ based on this approach and using the first few cumulants can be written as:

$$
f_{\tilde{I}_{A}}(y) \simeq f_{n d}(y)\left[1+\frac{\tilde{\kappa}_{3}\left(I_{A}\right)}{6} H_{3}(y)+\frac{\tilde{\kappa}_{4}\left(I_{A}\right)}{24} H_{4}(y)+\frac{\tilde{\kappa}_{3}^{2}\left(I_{A}\right)}{72} H_{6}(y)\right]
$$


where $\tilde{I}_{A}=\frac{I_{A}-\kappa_{1}\left(I_{A}\right)}{\kappa_{2}^{1 / 2}\left(I_{A}\right)}$ which is the standardized $I_{A}, f_{n d}(y)$ is the standard Normal $\mathrm{PDF}$, and $\tilde{\kappa}_{m}$ is the $m$ th standardized cumulants which is equal to $\frac{\kappa_{m}}{\kappa_{2}^{m / 2}}$ for $m \geq 2$ and 0 for $m=1 . H_{m}(y)$ are the Hermite polynomials which are defined by

$$
H_{m}(y)=(-1)^{m} f_{n d}(y)^{-1} \frac{d^{m}}{d y^{m}} f_{n d}(y)
$$

The (outage) interference probability can be calculated as

$$
P\left(I_{A} \geq I_{t h}\right)=P\left(\tilde{I}_{A} \geq \tilde{I}_{t h}\right)=\int_{\tilde{I}_{t h}}^{\infty} f_{\tilde{I}_{A}}(y) d y
$$

where $\tilde{I}_{t h}=\frac{I_{t h}-\kappa_{1}\left(I_{A}\right)}{\sqrt{\kappa_{2}\left(I_{A}\right)}}$.

It is important to highlight that while the Edgeworth series expansion is an asymptotic expansion for the PDF, the finite Edgeworth series should be applied with some caution: it is applicable for moderately-skewed distributions only. Conditions under which the Edgeworth finite approximation can be used are discussed in [94].

\subsubsection{Shifted Lognormal}

If the distribution of the aggregate interference has a positive skewness, $I_{A}$ can be approximated by a lognormal random variable. An enhanced version of the lognormal approximation, called shifted lognormal approximation, is proposed in [95], and used in [12] to approximate the distribution of the aggregate interference power. The shifted lognormal approximation is a three-parameter approximation. These parameters are obtained from the first three cumulants. To implement this approximation for the aggregate interference considered in this thesis, let $Z$ denotes the shifted lognormal random variable whose PDF can be written as

$$
f_{Z}(z)=\frac{1}{s(z-b) \sqrt{2 \pi}} e^{-(\ln (z-b)-u)^{2} / 2 s^{2}}, z>b
$$


where $s, b$, and $u$ can be calculated as follows:

$$
\begin{aligned}
& s^{2}=\ln \tau \\
& u=\frac{1}{2} \ln \frac{\kappa_{2}\left(I_{A}\right)}{\tau(\tau-1)} \\
& b=\kappa_{1}\left(I_{A}\right)-\sqrt{\frac{\kappa_{2}\left(I_{A}\right)}{\tau-1}} \\
& \tau=\left[v+\sqrt{v^{2}-1}\right]^{1 / 3}+\left[v-\sqrt{v^{2}-1}\right]^{1 / 3}-1, \\
& v=1+\frac{1}{2} \rho^{2} \\
& \rho=\frac{\kappa_{3}\left(I_{A}\right)}{\left[\kappa_{2}\left(I_{A}\right)\right]^{3 / 2}} .
\end{aligned}
$$

Then, the (outage) interference probability can be approximated as

$$
P\left(I_{A} \geq I_{t h}\right) \simeq Q\left(\frac{\ln \left(I_{t h}-b\right)-u}{s}\right)
$$

\subsubsection{General Remarks on the Approximations}

As an alternative approximation approach, cumulants can be used to calculate the moments. Then, moments-based approximations or bounds can be applied to approximate the distribution of $I_{A}[78]$.

Generally, [16] highlights some helpful guidelines for the approximation of the distribution for the aggregate interference which can be summarized as follows. The fading distribution dictates the approximating distribution of the aggregate interference. If the fading distribution in the aggregate interference decays polynomially, then the distribution of the aggregate interference should be approximated by a polynomially decaying distribution (like a lognormal distribution). However, if the fading distribution decays exponentially, then an exponentially decaying distribution (like a Gamma distribution) could be used as an approximating distribution. 


\subsubsection{Approximation and Simulation Results for the Distribution of $I_{A}$}

Fig. 5.1 and Fig. 5.2 compare the the performance of different approximating distributions with respect to the Monte Carlo simulation for fading and non-fading scenarios, respectively. Edgeworth series expansion, shifted lognormal and Gamma approximations work well for the non-fading case. However, if we go deep in the upper tail, the Edgeworth series approximation deviates. The performance of the shifted lognormal and Gamma approximations continue to provide good approximations. Introducing shadow fading increases the skewness of the distribution of $I_{A}$ and makes it a heavy tailed distribution. As a result, the Edgeworth series expansion fails to approximate the distribution; it generates a PDF with negative values, therefore, we exclude it from Fig. 5.2. The Gamma distribution also does not provide a good approximation when the distribution of $I_{A}$ has a heavy tail. The shifted lognormal distribution, on the other hand, provides an acceptable approximation of the upper tail of the distribution of $I_{A}$, as shown in Fig. 5.2. In this thesis, we focus more on the interference probability as the metric for the spectrum sharing; therefore, the upper tail of the CCDF is more relevant than the body.

\subsection{A Cumulant-Based Investigation of the Impact of Sec- ondary Users' Field Size on Spectrum Sharing Opportu- nities}

This section revisits the effect of the field (network) size on spectrum sharing opportunities, which is discussed preliminarily in Chapter 4. 


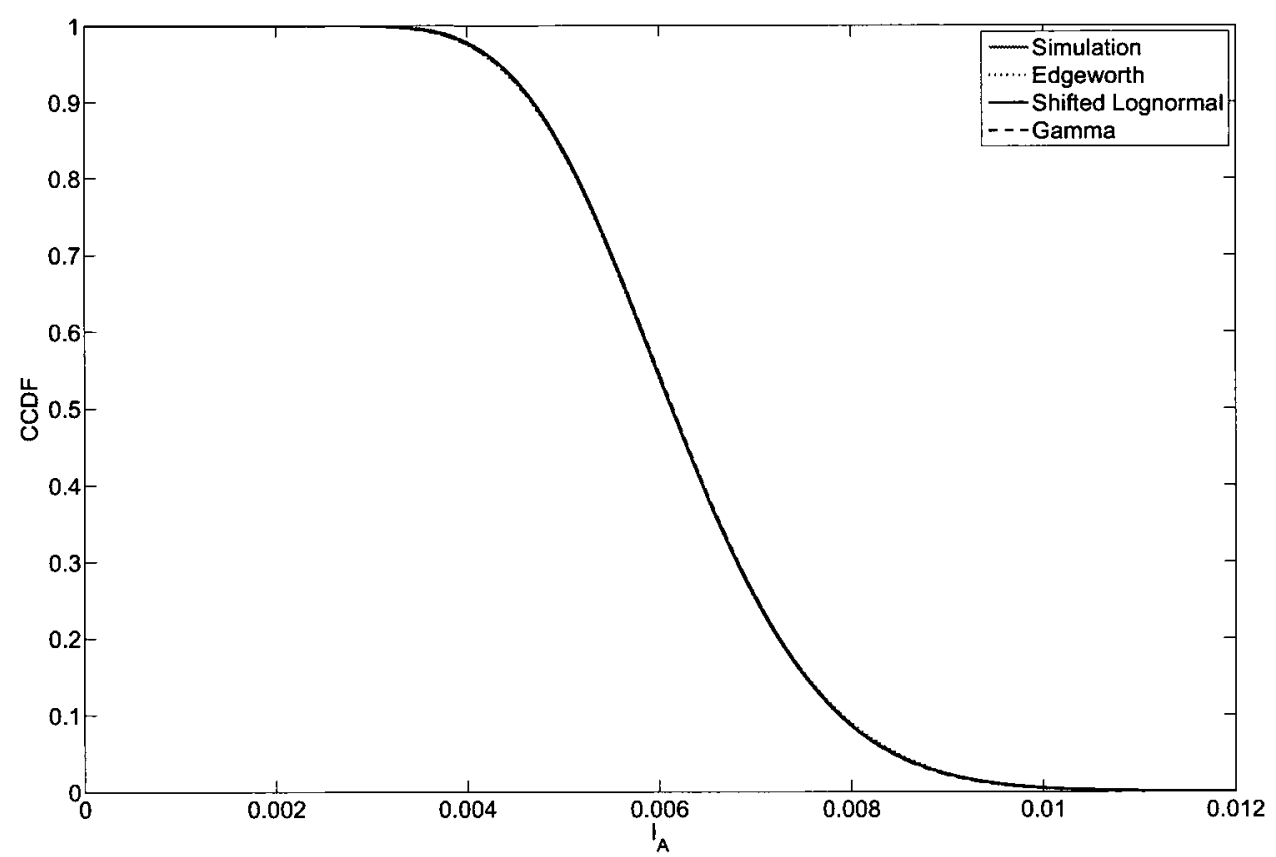

(a)

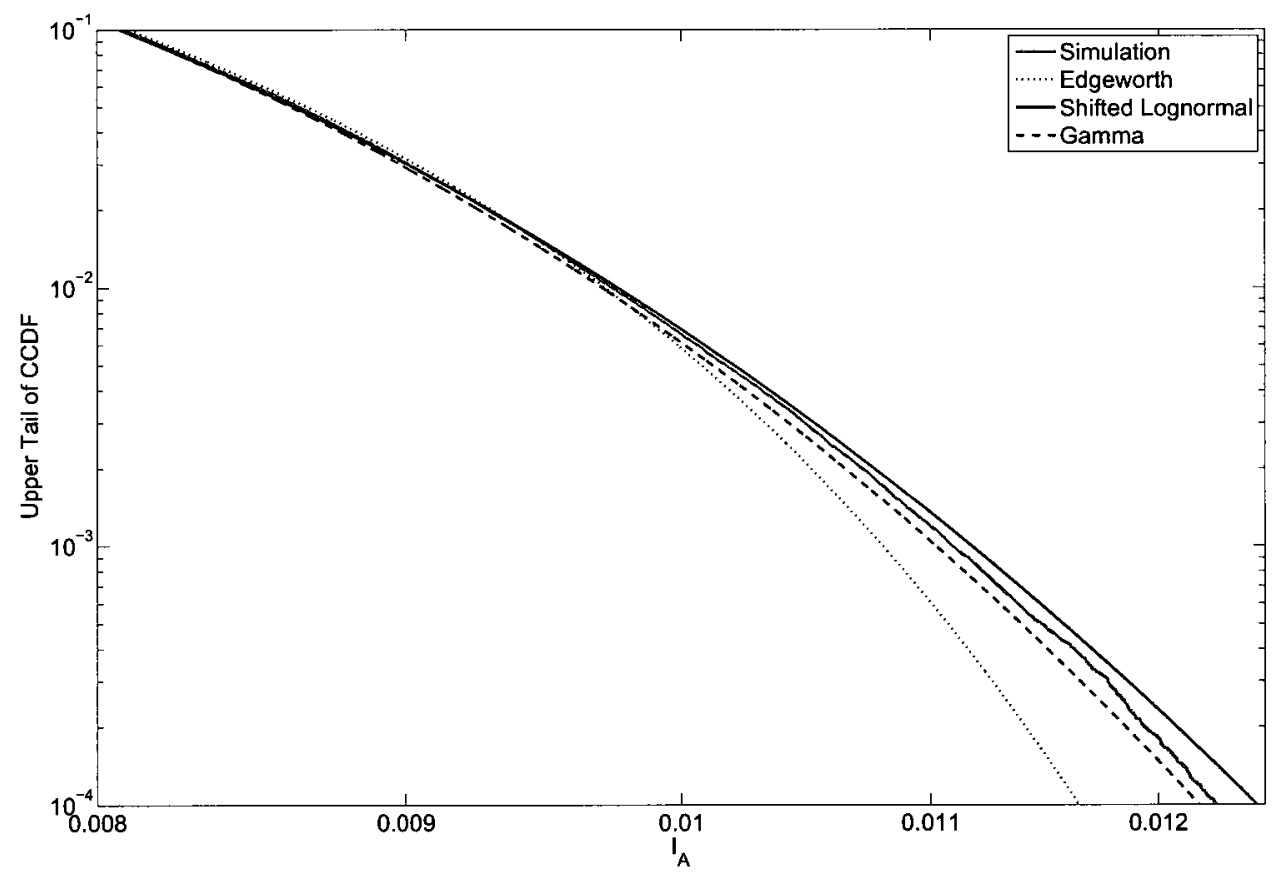

(b)

Figure 5.1: The CCDF of $I_{A}\left(r_{o}=10 \mathrm{~m}, L=1000 \mathrm{~m}, n=3, \lambda=0.01\right.$ node $/ \mathrm{m}^{2}$, $\theta=2 \pi$, no multipath fading, no shadow fading). (a) The CCDF on a linear scale. (b) The upper tail of the CCDF (log-log scale). 


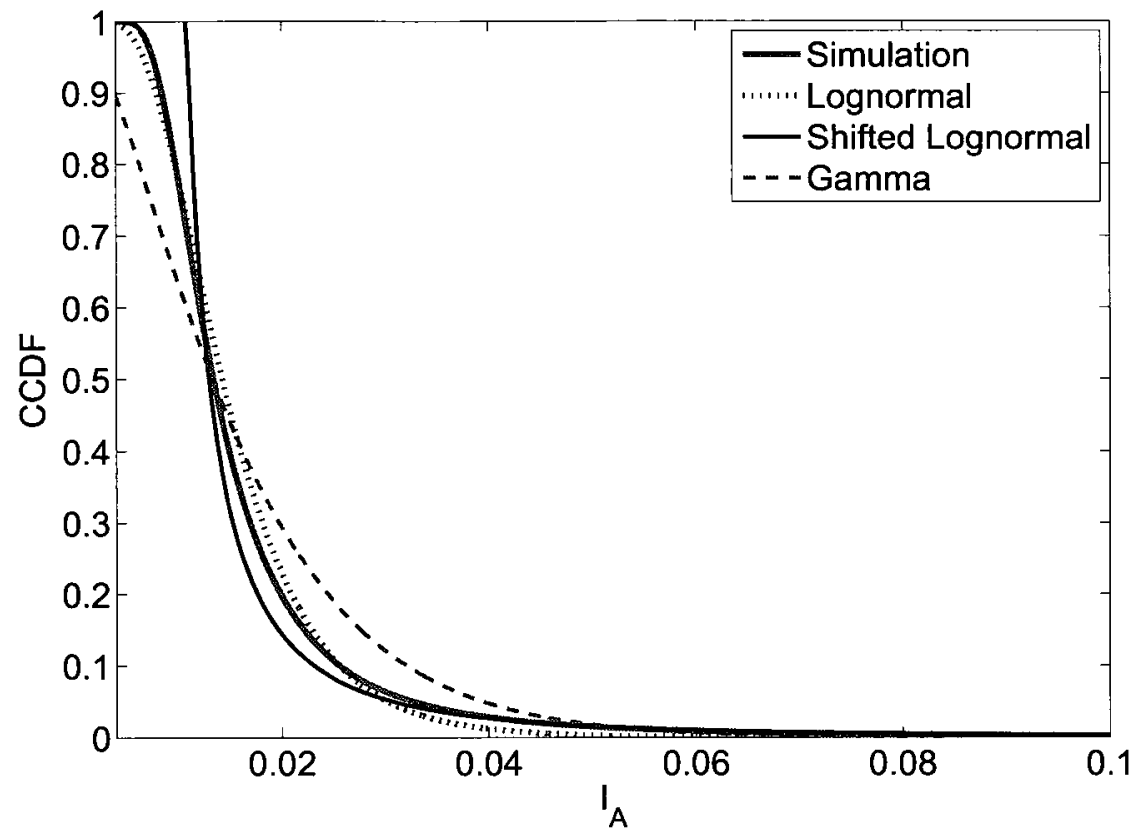

(a)

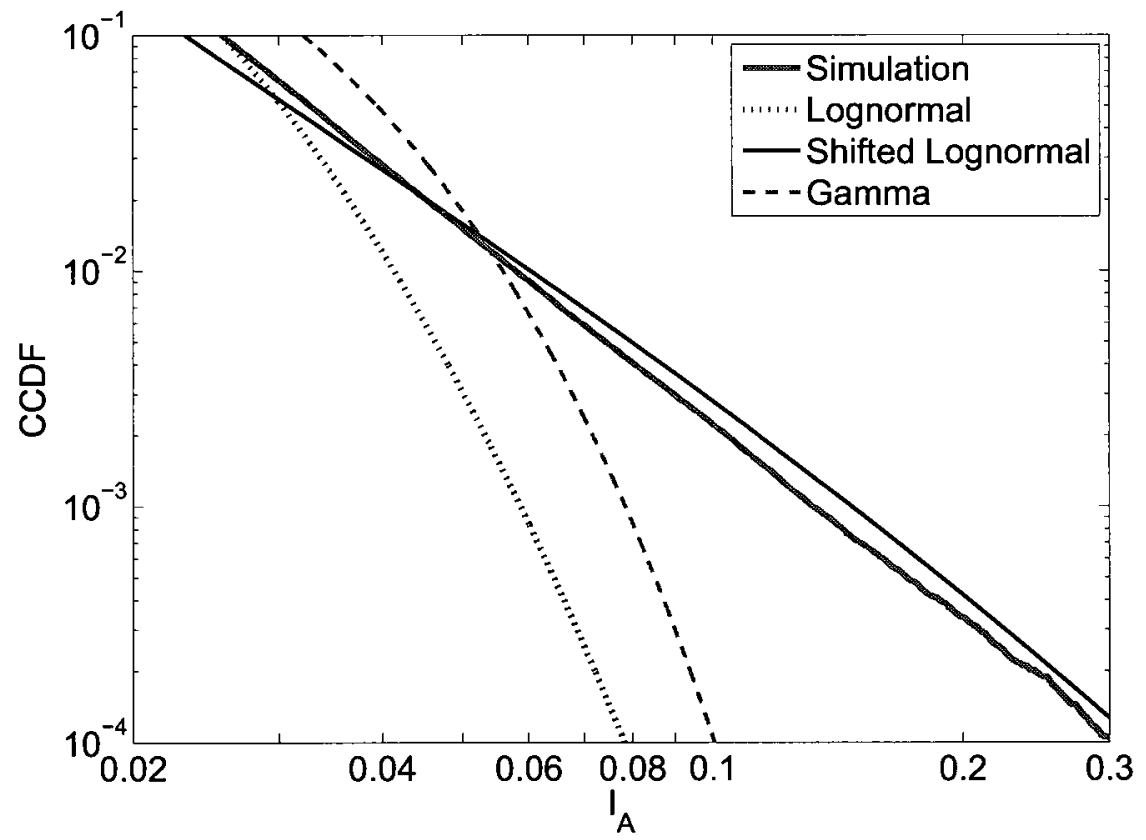

(b)

Figure 5.2: The CCDF of $I_{A}\left(r_{o}=10 \mathrm{~m}, L=1000 \mathrm{~m}, n=3, \lambda=0.01 \mathrm{node} / \mathrm{m}^{2}\right.$, $\theta=2 \pi$, Rayleigh fading, $6 \mathrm{~dB}$ shadow fading). (a) The CCDF on a linear scale. (b) The upper tail of the CCDF (log-log scale). The curve for the Edgeworth approximation is excluded because it fails to generate a valid CCDF curve for heavily skewed distributions. We show a lognormal approximation instead. 


\subsubsection{Objective and Approach}

Works such as $[9,12,17,18]$ study the effect of various system parameters on the harmful interference metric. However, a system parameter that has not received much attention is the spatial size of the field of secondary users. In most studies, the spatial size of the field is assumed to be infinite.

One might argue that asymptotic results obtained for infinite fields may be used as bounds for finite fields. However, since the spectrum sharing is opportunistic, using results developed for an infinite field may end up being too pessimistic leading to missing spectrum sharing opportunities. This concern about the applicability of the results of infinite fields could be addressed properly by studying the behavior of the harmful interference metric, and hence the spectrum sharing opportunities, with respect to changes in the field size.

In this section, we investigate the behavior of the cumulants, and hence the aggregate interference power, with respect to changes in the network size for various fading distributions. In this thesis, we consider a two-dimensional field but without assuming that the field is infinite; rather we consider a general setup in which the infinite field is a special case. We use the interference probability as the harmful interference metric. We study how the interference probability and, correspondingly, the spectrum sharing opportunities, would change with changes in the field size. The study shows that in some cases an increase in the field size may eliminate spectrum sharing opportunities; however, our study also demonstrates that there are other cases where the spectrum sharing opportunities are not affected by the increase in the field size even if the field size grows to infinity. Moreover, it is observed that while the reduction in the field size may open spectrum sharing opportunities in some cases; it does not in some other cases.

The rest of this section is organized as follows. First, Subsection 5.6.2 discusses 
the effect of the field size on the cumulants of $I_{A}$. Then, the effect of the field size on spectrum sharing is addressed in Subsection 5.6.3.

\subsubsection{Effect of Spatial Size of the Field and SU-TXs Density on the Cu- mulants}

As shown before, the $m$ th cumulant of $I_{A}$ can be expressed as

$$
\kappa_{m}\left(I_{A}\right)=N_{\mathrm{eff}}(m) g\left(r_{o}\right)^{m} \tilde{\mu}_{m}(X)
$$

where

$$
\begin{gathered}
N_{\mathrm{eff}}(m)=\lambda A_{\mathrm{eff}}(m), \\
A_{\mathrm{eff}}(m)=\frac{1}{2} \theta\left[r_{\mathrm{eff}}^{2}(m)-r_{o}^{2}\right] \\
r_{\mathrm{eff}}(m)=\hat{r} \sqrt{1+\frac{2}{m n-2}\left(1-\left[\frac{\hat{r}}{r_{o}+L}\right]^{m n-2}\right)}, \\
\hat{r}=\max \left(\min \left(r_{c}, r_{o}+L\right), r_{o}\right) .
\end{gathered}
$$

The spatial size of the field is controlled by $L, r_{o}$, and $\theta$. The changes in $L$ affect $r_{\text {eff }}$, and hence $A_{\text {eff }}$ and $\kappa_{m}$. However, this effect is limited. As $L$ increases, $r_{\text {eff }}$ increases but it tends to a limiting value regardless of further increase in $L$. As $m$ or $n$ increases, $r_{\text {eff }}$ approaches the limiting value faster. The effect of changes in $L$ is significant only for lower-order cumulants and for $L$ closer to or less than $\max \left(r_{c}, r_{o}\right)$. As $L$ approaches the value of $\max \left(r_{c}, r_{o}\right)$, its effect becomes weaker and it will be negligible when $\left(\frac{\max \left(r_{c}, r_{o}\right)}{r_{o}+L}\right)^{m n-2} \ll 1$.

As an example, Fig. 5.3 shows that the limiting value for $\kappa_{1}$ is almost reached when $L>100 \mathrm{~m}$ for a field with no exclusion region $\left(r_{o}=0\right)$. However, $\kappa_{2}$ is almost at the limiting value when $L>2 \mathrm{~m}$. The higher-order cumulants tend faster (at lower values of $L$ ) to the limiting values. Therefore, doubling $L$ or even expanding it 


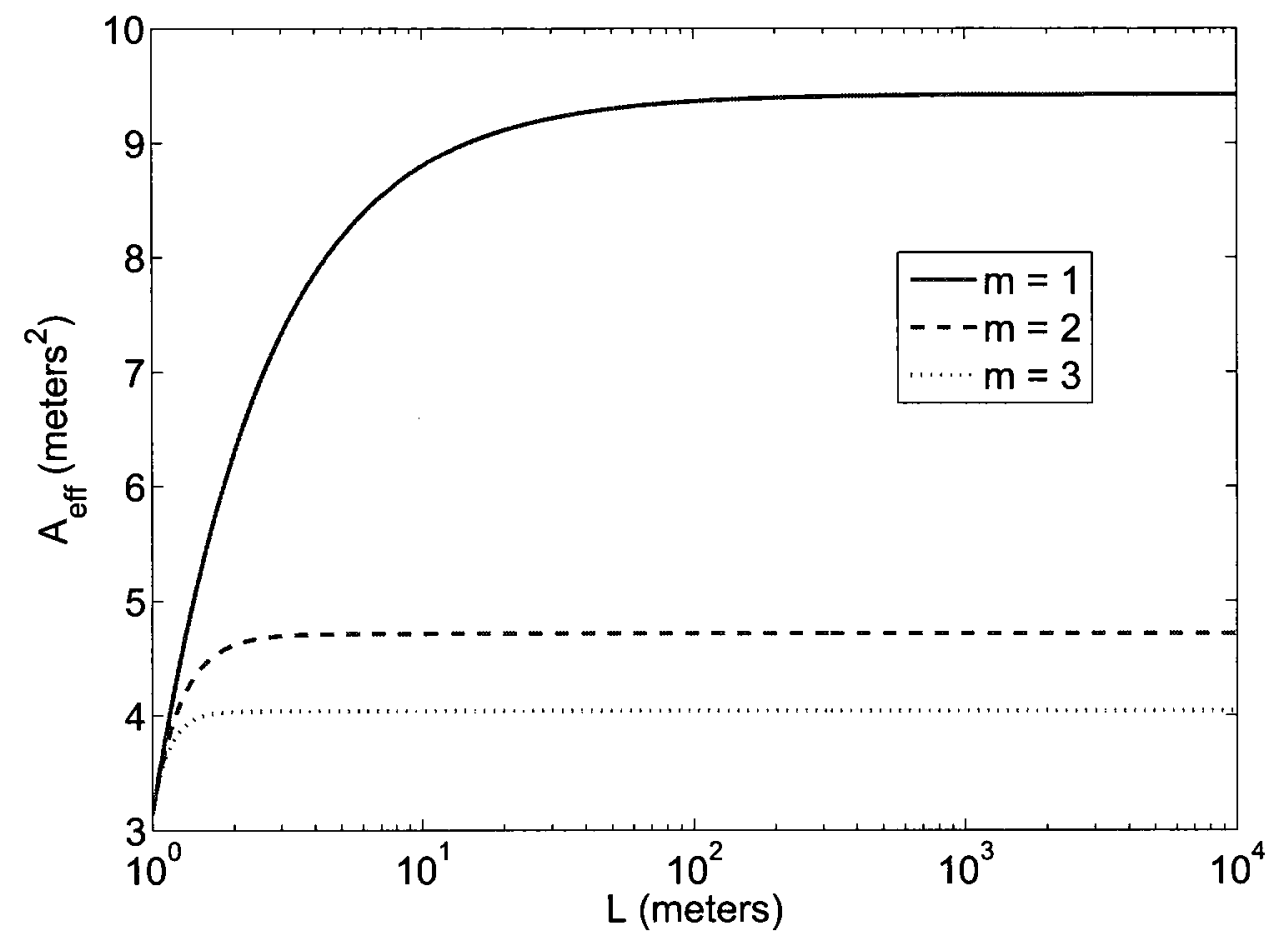

Figure 5.3: The effect of $L$ on $A_{\text {eff }}$, and hence on $\kappa_{m}$, for the case of no exclusion region around the PU-RX $\left(r_{c}=1\right.$ meter, $\theta=2 \pi$, and $\left.n=3\right)$.

to infinity has a negligible (or practically no) effect on $\kappa_{m}$ provided that $\kappa_{m}$ is almost at the limiting value.

If there is an exclusion region, e.g., $r_{o}=10 \mathrm{~m}$, then the limiting value will be almost reached when $L>1000 \mathrm{~m}$ for the first cumulant and $L>20 \mathrm{~m}$ for the second cumulant. A smaller $L$ will be required to closely approach the limiting value for higher cumulants, as seen in Fig. 5.4.

Interestingly, the value of $r_{\text {eff }}$ tends to $\max \left(r_{c}, r_{o}\right) \sqrt{1+\frac{2}{m n-2}}$ as $L \rightarrow \infty$. From this, we may conclude that $\kappa_{m}$ is controlled mainly by the region which is close to the victim receiver. The dominant region for $\kappa_{1}$ (the average) is wider than that for $\kappa_{2}$ (the variance). It shrinks as $m$ increases. On the other hand, this dominant region expands as $r_{o}$ (the exclusion region) increases.

The exclusion region has an effect on $A_{\text {eff }}$ of an order of $r_{o}^{2}$ (provided that $r_{o}>r_{c}$ ). Moreover, $r_{o}$ affects the value of $\tilde{\mu}_{m}\left(I_{r_{o}}\right)$ by an order of $r_{o}^{-m n}$. Therefore, the net effect 


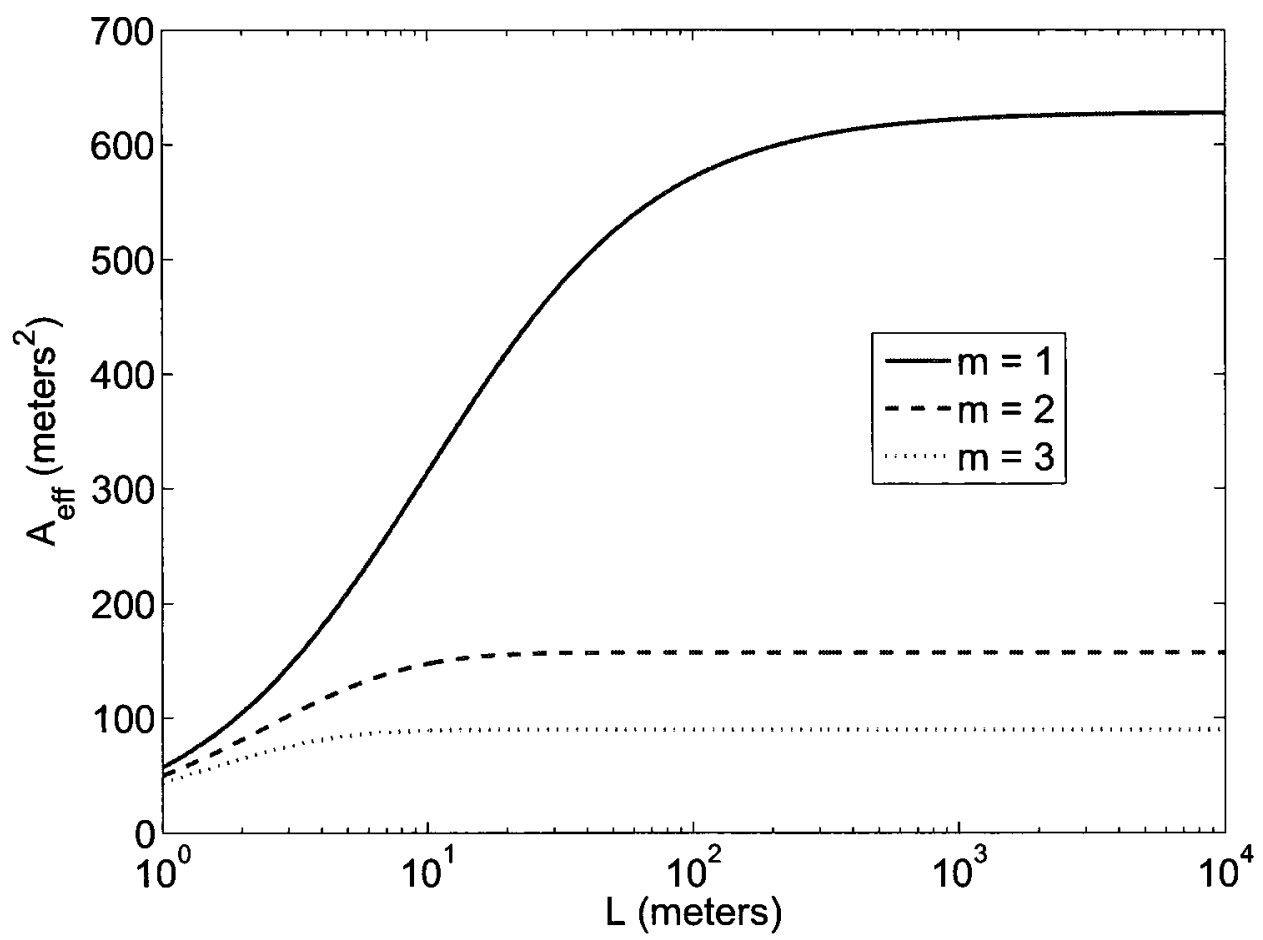

Figure 5.4: The effect of $L$ on $A_{\text {eff }}$, and hence on $\kappa_{m}$, for the case of an exclusion region of $r_{o}=10$ meters $\left(r_{c}=1\right.$ meter, $\theta=2 \pi$, and $\left.n=3\right)$.

of $r_{o}$ on $\kappa_{m}$ is in the order of $r_{o}^{2-m n}$, which suggests that increasing the value of $r_{o}$ is an effective way to lower the aggregate interference. However, increasing $r_{o}$ may contradict the performance objectives of the wireless network $[10,15,63]$. Therefore, an optimal tradeoff is to be found.

Regarding the effect of the active node density $(\lambda)$, it has a linear effect on all cumulants. Therefore, it is one of the important parameters that could be used to control the level of interference at the PU-RX.

Similarly, $\theta$ has a linear effect on the cumulants. However, it is limited to the range $[0,2 \pi]$. It may reflect the effectiveness of using a directional antenna at the PU-RX.

The effect of the increase in the network size (spatial size and node density) on the mean of the aggregate interference is considered in [57] and [7]. Moreover, the 
effect on the variance is discussed in $[21]^{1}$. However, to the best of our knowledge, this thesis is the first to address the effect of the spatial size on all cumulants of the aggregate interference. In this chapter, we don't focus on the effect of $r_{o}$ on $I_{A}$ because it has been the subject for other papers, such as [10], [15] and [63].

\subsubsection{Effect of Field Size on Spectrum Sharing Opportunities}

\subsubsection{Spectrum Sharing Opportunities}

If the condition in (3.6) is satisfied, we consider a spectrum sharing opportunity exists. Therefore, for a certain value of $I_{t h}$, the interference probability $\left(P_{\text {int }}=P\left(I_{A} \geq I_{t h}\right)\right)$ will be compared to $\beta$. If $P_{\text {int }}$ is less than or equal to $\beta$, then the aggregate interference of the secondary network is considered to be non-harmful to the PU. In this case, the secondary network is considered to be operating in a non-interfering region. On the other hand, the secondary network operates in an interfering region if it causes harmful interference to the PU.

If the secondary network operates in an interfering region, then it may access the spectrum of the PU during the absence of the PU. However, when the PU starts using the spectrum, then the secondary network has to leave the spectrum or to change its transmission parameters to move to the non-interfering region. For the secondary network to be able to sense the PU and to perform a proper action accordingly, it should employ a technology such as a "cognitive radio" $[5,6]$.

On the other hand, if the secondary network operates in the non-interfering region, then it can concurrently share the spectrum with the PU. It is possible that the secondary network operations will be in the non-interfering region under all practically possible scenarios. In this case, the secondary network could share the spectrum with the PU without the need to implement any spectrum sensing technique or cognition

\footnotetext{
${ }^{1}$ The divergence of the aggregate interference power for an infinite two-dimensional field is also discussed in $[55,56]$.
} 
related function.

\subsubsection{Field Size and Spectrum Sharing Opportunities}

In this chapter, a discussion of the effect of the field size on spectrum sharing opportunities is based on approximating the distribution of $I_{A}$ by a shifted lognormal; see Chapter 5.5 for more details. Therefore, the interference probability can be written as

$$
P\left(I_{A} \geq I_{t h}\right) \simeq Q\left(\frac{\ln \left(I_{t h}-b\right)-u}{s}\right) .
$$

Expressions for $b, u$ and $s$ are given in Section 5.5.2 in terms of the cumulants of $I_{A}$. Fig. 5.5 reflects the effect of the field size on spectrum sharing opportunities. This figure has a straight line that corresponds to a certain value of $\beta$. This line divides the figure into two main regions: interfering and non-interfering regions.

If $\beta$ (e.g., 0.1 ) is the maximum interference probability that a PU can tolerate, then the aggregate interference generated by the secondary network is not considered to be harmful if the interference probability is less than $\beta$. Therefore, a non-interfering region in Fig. 5.5 corresponds to the part of the figure below the straight line of $10^{-1}$. In this case, the spectrum of the PU can be shared with the secondary network.

If the secondary network operates in the upper part of Fig. 5.5, i.e., the interfering region, the secondary network could utilize the spectrum during the absence of the PU. If the PU is present and active, then it is possible for the secondary network to access the spectrum provided that it has adapted its operation parameters, and has moved to the non-interfering region.

There are three curves in Fig. 5.5; each curve corresponds to a certain value of $I_{t h}$. For the curve corresponding to $I_{t h}=0.009,{ }^{2}$ the secondary network does not cause

\footnotetext{
${ }^{2} \mathrm{All}$ values of $I_{t h}$ mentioned here and in Fig. 5.5 are normalized with respect to the deterministic parts of $I_{A}$, e.g., transmit power and antenna gains, which are not of interest in this discussion. The values of $I_{t h}$ are properly chosen from the corresponding CCDF curve; see for example Fig. 5.1.
} 


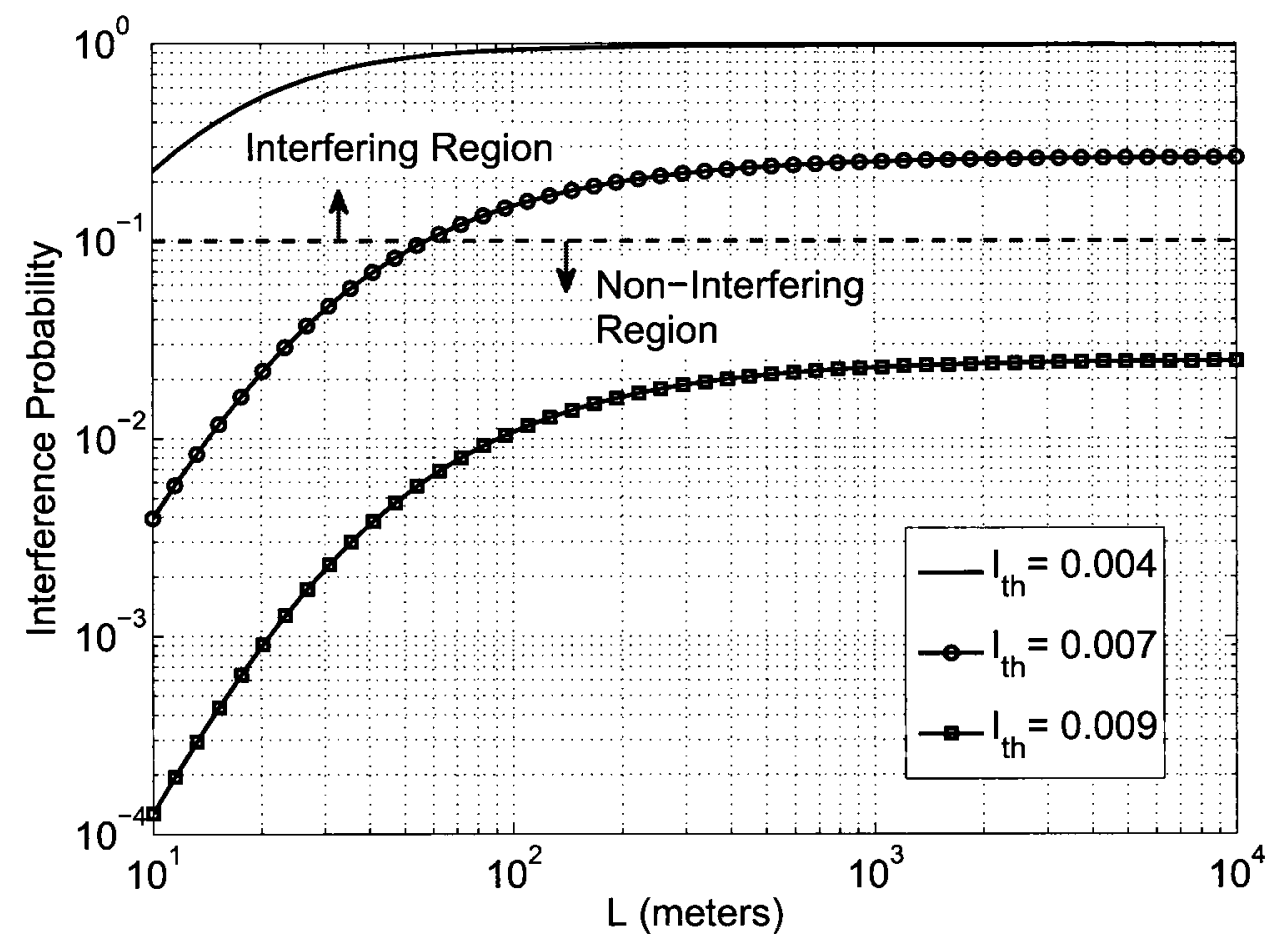

Figure 5.5: Field size and spectrum sharing opportunities $\left(r_{o}=10 \mathrm{~m}, n=3, r_{c}=1\right.$ $\mathrm{m}, \theta=2 \pi, \lambda=0.01$ node $/ \mathrm{m}^{2}$, no multipath fading, no shadow fading). The dashed line corresponds to $P_{i n t}=\beta=0.1$, which divides the figure into two regions: a noninterfering region (the lower part of the figure), and an interfering region (the upper part of the figure). The values of $I_{t h}$ are properly chosen from the corresponding CCDF curve; see for example Fig. 5.1.

harmful interference towards the PU regardless of the field size, $L$. Therefore, the field expansion does not eliminate any spectrum sharing opportunity. For the curve corresponding to $I_{t h}=0.007$, on the other hand, the increase in $L$ may move the secondary network from the non-interfering region to the interfering region eliminating a spectrum sharing opportunity. From the same curve and starting with a field with a large $L$, we may also deduce that a reduction in the field size might lead to moving the secondary network from the interfering region to the non-interfering region which creates a spectrum sharing opportunity. For the curve corresponding to $I_{t h}=0.004$, a reduction in $L$ never moves the secondary network from the interfering region to the non-interfering region except when there is no transmitting node. Therefore, reducing 
the field size here does not create a spectrum sharing opportunity.

At this stage we can comment on the impact of the field size on the spectrum sharing opportunities. In summary, it has been shown that an increase in the field size may eliminate spectrum sharing opportunities. However, it is possible that the increase in the field size, even if the field size grows to infinity, does not affect the the spectrum sharing opportunities. Moreover, a reduction in the field size may open spectrum sharing opportunities. However, there are some cases where the reduction in the size of the field does not help in opening any spectrum sharing opportunities.

Regarding the applicability of asymptotic results obtained for a field of an infinite size to the case where the field size is finite, we can state the following: asymptotic results obtained for infinite fields can be applied for finite fields whose radial depth $(L)$ is much greater than the minimum distance between the field and the PU $\left(r_{o}\right)$. Otherwise, these asymptotic results will be too conservative and may lead to missing spectrum sharing opportunities.

In Fig. 5.5 where $r_{o}=10$ meters, the interference probability is almost constant as long as $L>1000$ meters. Fig. 5.6 shows curves for different values of $r_{o}$. From this figure, we can highlight some observations as follows. For lower $r_{o}$, the interference probability tends to a limiting value faster with respect to the increase in L. Furthermore, Fig. 5.6 reflects the importance of the exclusion region in creating spectrum sharing opportunities. A slight increase in $r_{o}$ could move the operation of the secondary network to the non-interfering region and create a spectrum sharing opportunity.

The value of $r_{o}$ can be properly designed during the pre-deployment stages of the secondary network. For an already deployed secondary network, it is possible to virtually control $r_{o}$ by a MAC protocol forcing nodes within a distance of $r_{o}$ from the primary user not to transmit. 


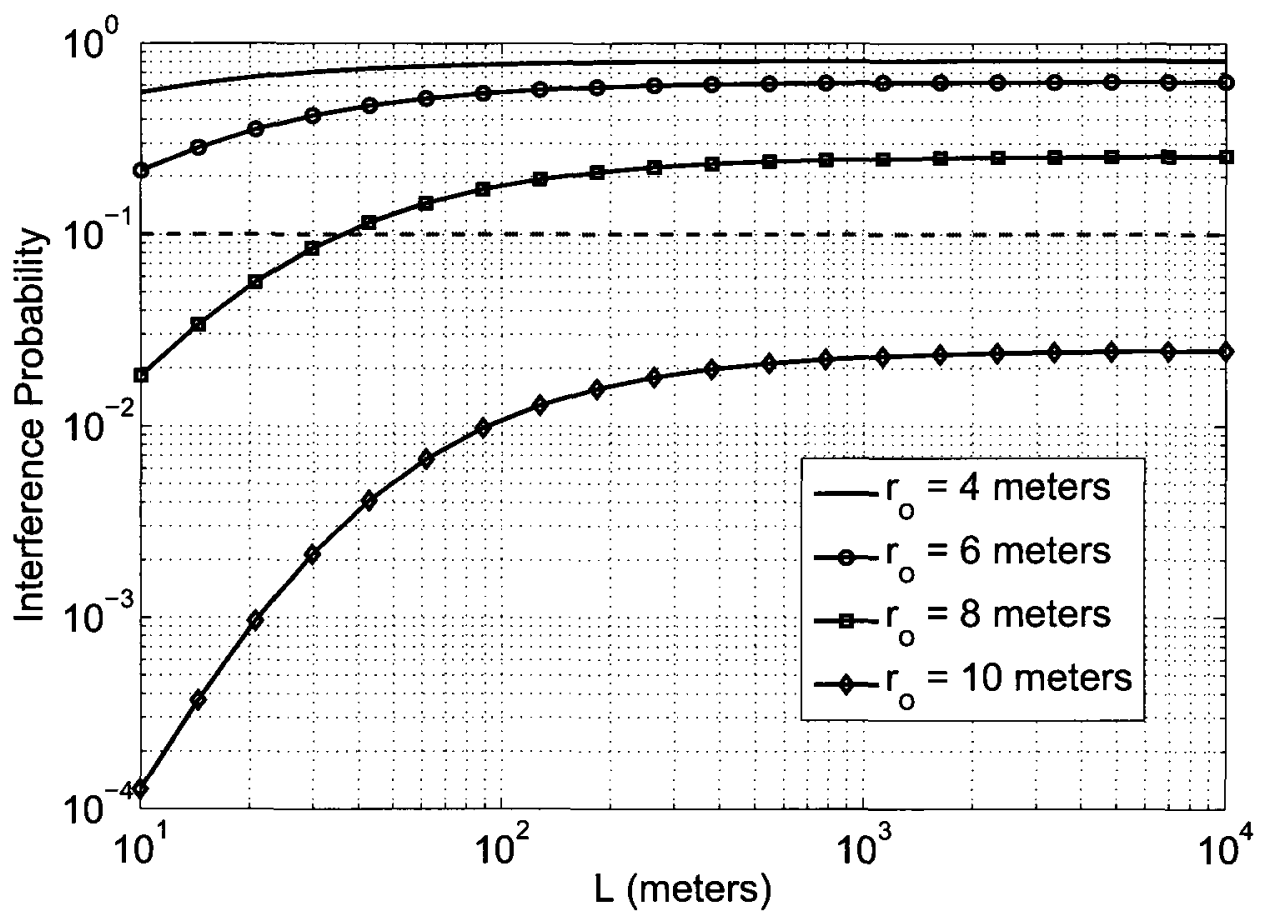

Figure 5.6: Field size and spectrum sharing opportunities under different values of $r_{o}$ ( $n=3, r_{c}=1 \mathrm{~m}, \theta=2 \pi, \lambda=0.01$ node $/ \mathrm{m}^{2}$, no multipath fading, no shadowing, $I_{t h}$ $=0.009$ ). The dashed line corresponds to $P_{\text {int }}=\beta=0.1$, which divides the figure into two regions: a non-interfering region (the lower part of the figure), and an interfering region (the upper part of the figure).

\subsection{Conclusions}

We characterized the aggregate interference power generated by a secondary network through a cumulant-based approach. We introduced a simple yet comprehensive method to calculate the cumulants. Our method is applicable for finite and infinite secondary networks, and is flexible to encompass different system and propagation parameters including large-scale fading, small-scale fading, and composite fading. Moreover, we presented some cumulant-based approximations of the distribution of the aggregate interference power. The shifted lognormal distribution shows a better match for the upper tail of the distribution of the aggregate interference power especially when the skewness is high. Since the upper tail of the distribution of the 
aggregate interference power is more relevant to my discussions on spectrum sharing, the shifted lognormal distribution is used as the approximating distribution in this thesis. We discussed the behavior of these cumulants with respect to changes in the network size and fading distributions. An important contribution of this chapter is the study of the impact of the field size of secondary users on the aggregate interference power, and hence on spectrum sharing opportunities. We showed that an increase in the field size may eliminate the spectrum sharing opportunities. However, we also showed that there are some cases where the spectrum sharing opportunities are not reduced by the increase in the field size even when the field size grows to infinity. It was demonstrated that asymptotic results obtained for an infinite field could be applied for a finite field whose radial depth is much greater than the minimum distance between the field and the primary user. Otherwise, these asymptotic results will be too conservative and may lead to missing spectrum sharing opportunities. 


\section{Chapter 6}

\section{The Gaussian Convergence of the Distribution of the Aggregate Interference Power}

As the number of interfering nodes in a wireless network increases, there might be a tendency to approximate the distribution of the aggregate interference power by a Gaussian random variable given that the individual interference signals are independent. However, some observations in the literature suggest that this Gaussian approximation is not valid, except under some specific scenarios. In this chapter, we cast these observations in a single mathematical framework and express the conditions for which the Gaussian approximation will be valid for the aggregate interference power generated by a Poisson field of interferers. Furthermore, we discuss the effect

of different system and channel parameters on the convergence of the distribution of the aggregate interference to a Gaussian distribution.

\subsection{Introduction}

It is common to characterize the interference in large wireless networks using a Poisson point process (PPP). Therefore, the aggregate interference can be considered as the sum of a large number of independent interference signals. Thus, there might be a tendency to approximate the aggregate interference power by a Gaussian random 
variable. However, this approximation is not valid, except under certain conditions. the authors in [11] consider the interference in a CDMA network and indicate that the distribution of the aggregate interference power from users in other cells is likely to be Gaussian if there is a large number of interfering users in the vicinity of the victim cell. The authors in [13] consider the aggregate interference in a CDMA network as well and show that the distribution of the aggregate interference power converges to a Gaussian distribution as the traffic measure (which can be related to the average number of interferers in a cell) goes to infinity. The authors in [14] indicate that the Central Limit Theorem (CLT) ${ }^{1}$ does not apply in the case where some of the interferers are dominant although the number of interferers may be large. It is indicated in [16] that the Gaussian distribution is a bad approximation for the distribution of the aggregate interference when the node density is low. Based on simulation results, [15] shows that the Gaussian approximation could be acceptable when there is a wide-enough exclusion region (with no interferers) around the victim receiver. It is also indicated in [48] that when the exclusion region is relatively small the distribution of the interference power has a heavy tail; therefore, the Gaussian distribution does not model the interference very well ${ }^{2}$.

Observing that the aggregate interference can be modeled as shot noise, discussions in [99] on the convergence of shot noise to a Gaussian random variable become relevant to our study. The authors in [99] proved that under certain conditions the shot noise converges in distribution to Gaussian when the intensity (density) of the underlying point process of the shot noise goes to infinity. However, no discussion has been given to the effect of the exclusion region on this convergence. In this chapter,

\footnotetext{
${ }^{1}$ The history of the CLT is summarized in [96].

${ }^{2}$ In the context of ultrawide bandwidth (UWB) systems, the validity of the Gaussian approximation for the multiuser interference in Time-Hopped UWB is discussed in [97,98]. However, the number of interferers is assumed to be deterministic. In contrast, this thesis uses PPP which accounts for the spatial distribution of the interferers and imposes a randomness in the number of interferers.
} 
we apply some of the results obtained in [99] to the case of the aggregate interference power in large wireless networks. We incorporate in the formulations the effect of the exclusion region and identify the rate of the Gaussian convergence with respect to the size of the exclusion region. Moreover, we discuss the effect of fading distributions, including the small-scale and large-scale fading, on this convergence.

The rest of this chapter is organized as follows. Section 6.2 establishes the mathematical framework to quantify how far away the distribution of the aggregate interference power is from a Gaussian distribution. Also, the same section discusses some system and channel parameters affecting the convergence to a Gaussian distribution. The effect of the fading distributions is addressed in Section 6.3. The chapter is concluded by some remarks in Section 6.4.

\subsection{Berry-Esseen Bound for the Distribution of $I_{A}$}

Our investigation of the Gaussianity of the distribution of $I_{A}$ is based on the BerryEsseen bound. This bound is explained in its basic form in Appendix A. Formulations for the Berry-Esseen bound when the underlying process is a stationary PPP are obtained in [99]. These formulations are extended in [100] by removing the requirement that a PPP is stationary. We apply the results in [99] and [100] to investigate the Gaussianity of the distribution of $I_{A}$.

Let $\tilde{\mu}_{A}$ and $\sigma_{A}$ be the mean and standard deviation of $I_{A}$, respectively. Let $F_{Z}(y)$ denote the cumulative distribution function (CDF) of $Z$ (where $Z=\frac{I_{A}-\tilde{\mu}_{A}}{\sigma_{A}}$ ), and $F_{N}$ denote the CDF of the standard normal distribution, i.e., $\mathcal{N}(0,1)$. Then, for all $y$

$$
\left|F_{Z}(y)-F_{N}(y)\right| \leq 2.21 \frac{\kappa_{3}\left(I_{A}\right)}{\left[\kappa_{2}\left(I_{A}\right)\right]^{\frac{3}{2}}}
$$

where $\kappa_{2}\left(I_{A}\right)$ and $\kappa_{3}\left(I_{A}\right)$ are the second and third cumulants of $I_{A}$, respectively. 
Equation (6.1) is a new formulation of the Berry-Esseen bound. The formulation of the Berry-Esseen bound in [99] is not explicitly presented in terms of cumulants. However, as shown in Appendix B, we equivalently expressed the Berry-Esseen bound using the second and the third cumulants.

Results obtained in Chapter 5, [48], or [58] can be used to find $\kappa_{2}\left(I_{A}\right)$ and $\kappa_{3}\left(I_{A}\right){ }^{3}$ Based on Chapter 5 , the $m$ th cumulant of $I_{A}$ can be written as

$$
\kappa_{m}\left(I_{A}\right)=N_{\mathrm{eff}}(m) g\left(r_{o}\right)^{m} \tilde{\mu}_{m}(X),
$$

where

$$
\begin{gathered}
N_{\text {eff }}(m)=\lambda \pi\left[r_{\text {eff }}^{2}(m)-r_{o}^{2}\right], \\
r_{\text {eff }}(m)=\max \left(r_{c}, r_{o}\right) \sqrt{\frac{m n}{m n-2}} .
\end{gathered}
$$

Here, $N_{\text {eff }}(m)$ virtually represents the average number of active nodes as seen by the victim receiver in an annular region of inner radius $r_{o}$ and an outer radius $r_{\text {eff }}$. The parameter $\tilde{\mu}_{m}(X)$ denotes the $m$ th raw moment of $X_{i}$, i.e., $\mathbb{E}\left[X_{i}^{m}\right]$. For convenience and since $X_{i}$ s are i.i.d., we omit the subscript $i$. Using (6.2) and (6.3) in (6.1), the Berry-Esseen bound for the distribution of $I_{A}$ can be expressed as

$$
\left|F_{Z}(y)-F_{N}(y)\right| \leq 2.21 \frac{N_{\mathrm{eff}}(3) \tilde{\mu}_{3}(X)}{\left[N_{\mathrm{eff}}(2) \tilde{\mu}_{2}(X)\right]^{\frac{3}{2}}} .
$$

There are three possible topologies with respect to the exclusion region: an exclusion region with $r_{o} \geq r_{c}$, no exclusion region $\left(r_{o}=0\right)$, and an exclusion region with $0<r_{o}<r_{c}$. We do not discuss the third topology here since results for the third

\footnotetext{
${ }^{3}$ It is worth highlighting that cumulants are derived by differentiating the logarithm of the characteristic function (or moment generating function) of $I_{A}$. There are different approaches in the literature to derive this characteristic function for a PPP. One approach is to use the conditional expectation as explained in $[48,53]$. Another approach is to use Campbell's theorem for a PPP as demonstrated in $[11,12,22]$.
} 
topology are bounded by the results of the first two topologies. However, results for the third topology can be easily obtained from (6.2), (6.3), and (6.4).

\subsubsection{Exclusion Region $\left(r_{o} \geq r_{c}\right)$}

For this topology, it can be shown from (6.3) and (6.4) that the Berry-Esseen bound yields $^{4}$

$$
\left|F_{Z}(y)-F_{N}(y)\right| \leq 2.21 \frac{2(n-1)^{\frac{3}{2}}}{3 n-2} \frac{1}{\sqrt{\lambda \pi r_{o}^{2}}} \frac{\tilde{\mu}_{3}(X)}{\left[\tilde{\mu}_{2}(X)\right]^{\frac{3}{2}}} .
$$

Remarks:

- It is observed from (6.5) that the bound is mainly controlled by the path-loss exponent, the active node density, the radius of the exclusion region, and the fading distribution.

- The active node density is an important parameter in the convergence of the distribution of $I_{A}$ to a Gaussian distribution. As $\lambda$ increases, the bound becomes smaller and the distribution of $I_{A}$ becomes closer to the Gaussian distribution; Figs. 6.1 and 6.2 show simulation results supporting this remark. Fig. 6.1 considers the histograms of the normalized $I_{A}$ for different values of $\lambda$. These histograms reflect the shape of the PDF of $I_{A}$ as compared to a Gaussian distribution. In Fig. 6.2 the comparison is performed among the CDFs. The rest of the figures shown in this chapter focus on CDFs, which are more relevant to the Berry-Esseen bound. It is observed from (6.5) that the rate of Gaussian convergence with respect to the increase in $\lambda$ is $\sqrt{\lambda}$, which agrees with the findings in [99].

- Similarly, as the exclusion region increases, the bound in (6.5) becomes smaller. Hence, the distribution of $I_{A}$ converges to a Gaussian distribution. Simulation

\footnotetext{
${ }^{4} \mathrm{~A}$ generalization of (6.5) for a $d$-dimensional space is considered in Appendix C.
} 


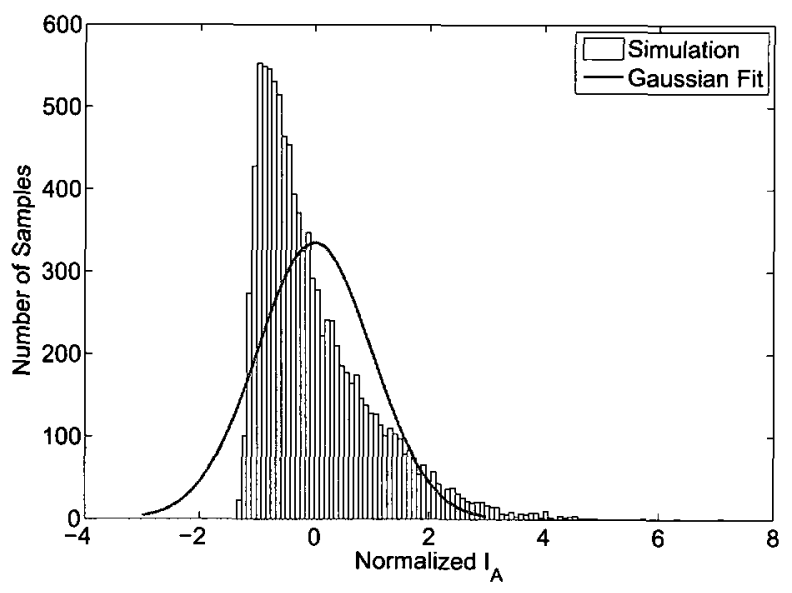

(a)

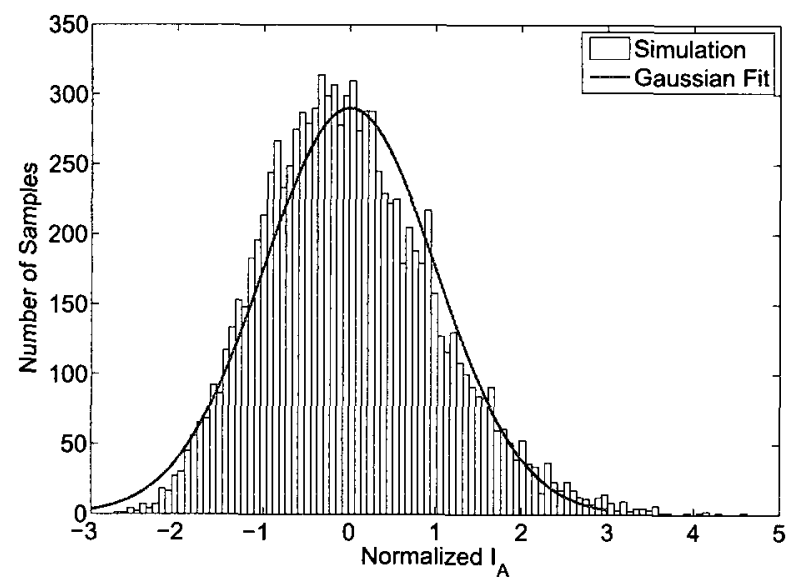

(b)

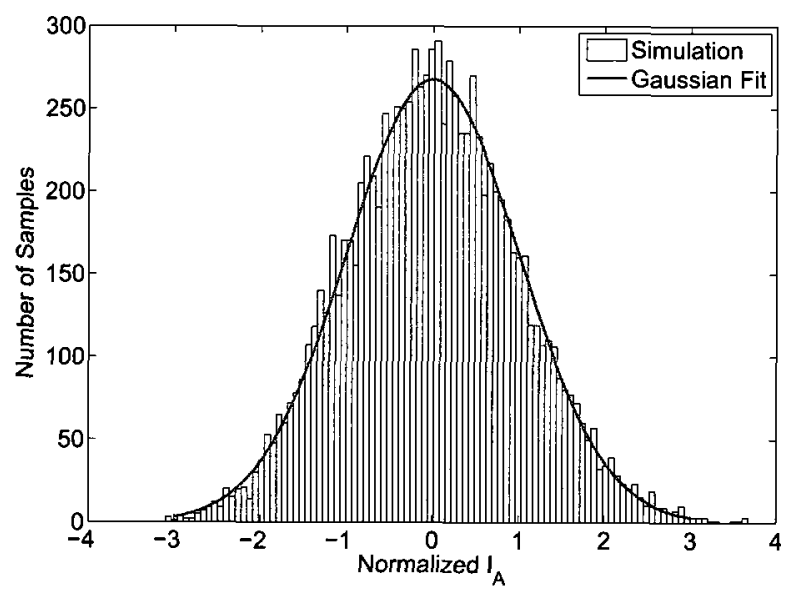

(c)

Figure 6.1: Monte-Carlo simulation-based histograms for the normalized $I_{A}$, i.e., $\frac{I_{A}-\tilde{\mu}_{A}}{\sigma_{A}}$, for different values of $\lambda\left(r_{o}=10\right.$ meters, $r_{c}=1$ meter, $n=3$, no multipath fading, and no shadow fading). (a) $\lambda=0.001$ nodes $/$ meter $^{2}$. (b) $\lambda=0.01$ nodes $/$ meter $^{2}$. (c) $\lambda=0.1$ nodes $/$ meter $^{2}$. 


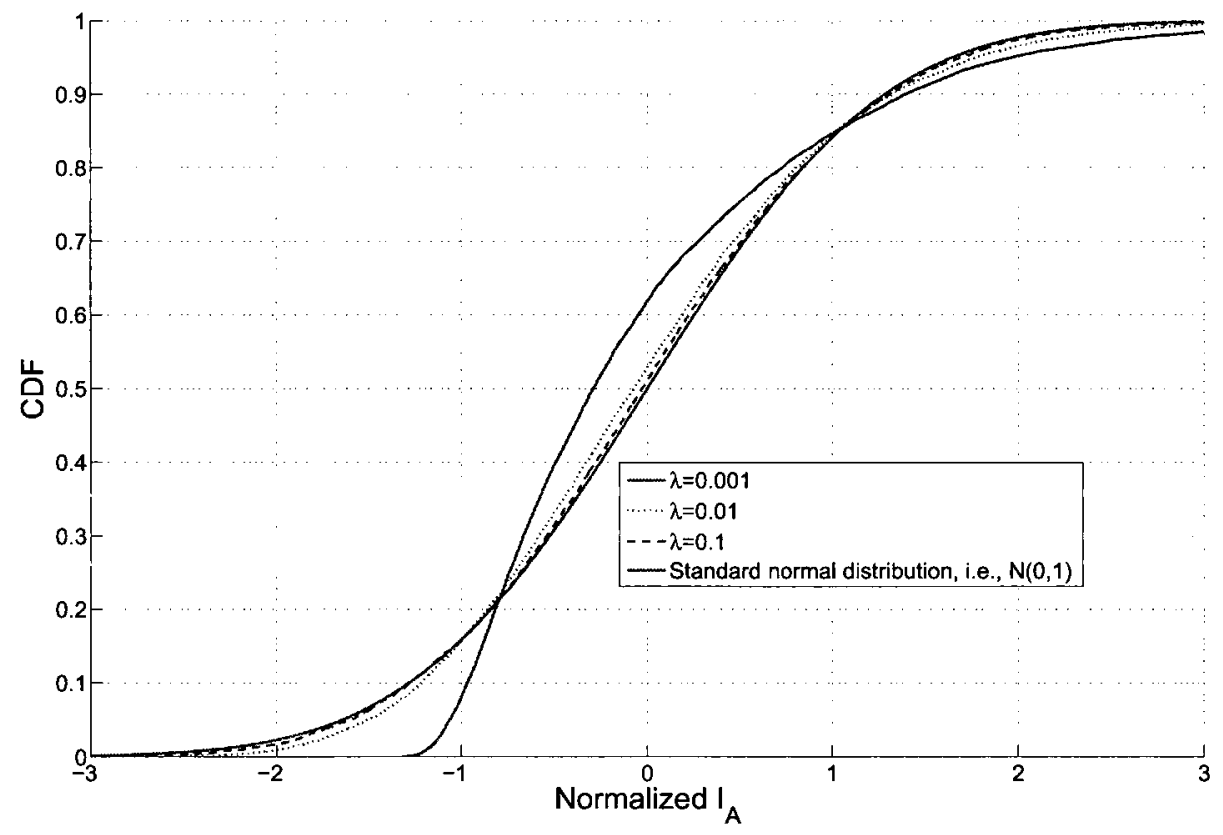

Figure 6.2: Monte-Carlo simulation-based CDF for the normalized $I_{A}$, i.e., $\frac{I_{A}-\tilde{\mu}_{A}}{\sigma_{A}}$, for different values of $\lambda\left(r_{o}=10\right.$ meters, $r_{c}=1$ meter and $\left.n=3\right)$.

results shown in Fig. 6.3 demonstrate this convergence. However, the convergence caused by increasing $r_{o}$ is faster than convergence caused by increasing $\lambda$. The rate of convergence with respect to the size of the exclusion region is $\sqrt{\pi r_{o}^{2}}$.

- An explanation for this convergence with respect to the increase in $\lambda$ and $r_{o}$ is as follows. What really matters for the convergence of the distribution of $I_{A}$ to Gaussianity is the number of dominant interferers around the victim receiver, not the total number of interferers in the field. The number of dominant interferers is controlled mainly by the active node density and an effective area around the victim receiver. As $\lambda$ increases, the number of dominant interferers increases. Similarly, as $r_{o}$ increases the effective area increases as well, hence the number of the dominant interferers increases. By virtue of the CLT, as the number of the dominant interferers increases, the distribution of $I_{A}$ converges to a Gaussian distribution. 


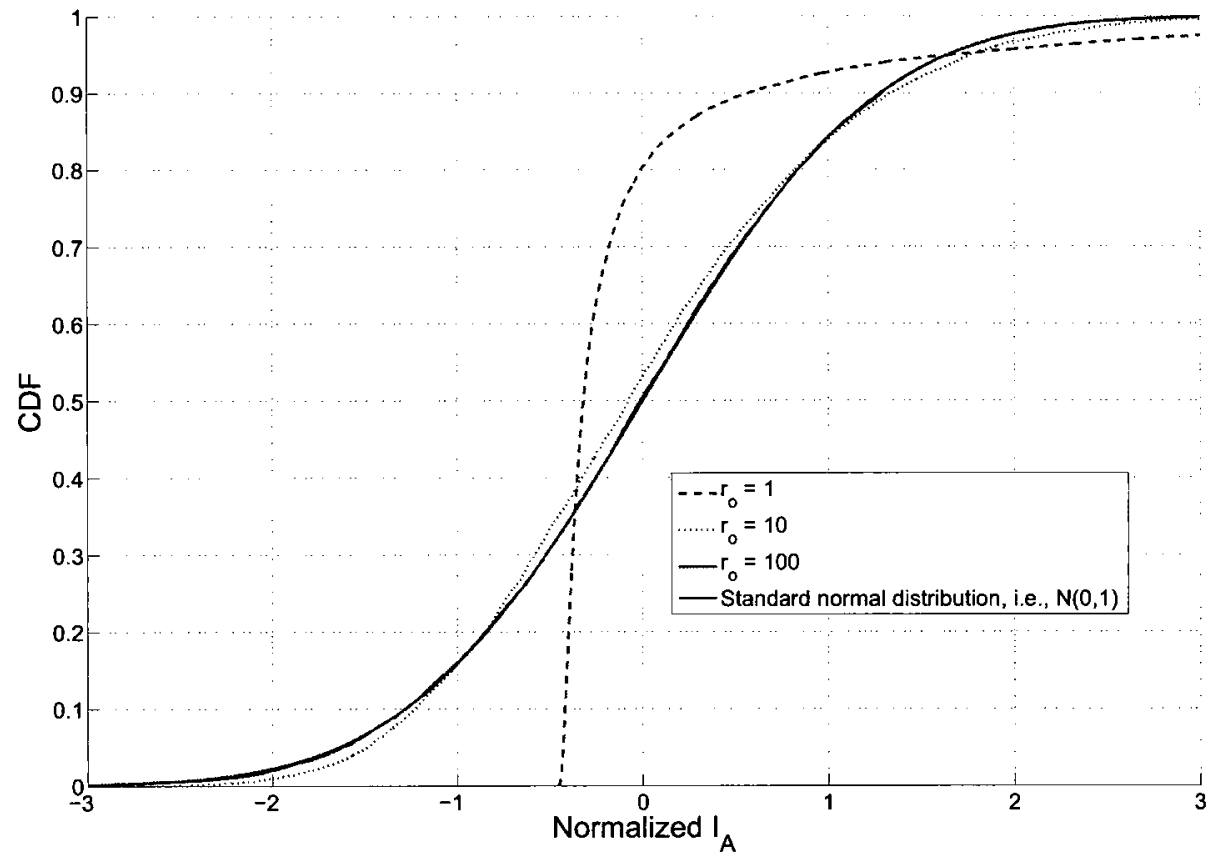

Figure 6.3: Monte-Carlo simulation-based CDF for the normalized $I_{A}$, i.e., $\frac{I_{A}-\tilde{\mu}_{A}}{\sigma_{A}}$, for different values of $r_{o}\left(\lambda=0.01\right.$ nodes $/$ meter $^{2}, r_{c}=1$ meter and $\left.n=3\right)$.

- The lower $n$ is, the better the convergence becomes. However, this effect on convergence is minor since the range of $n$ is practically limited. Assuming $n \in(2,6]$,

$$
0.5<\frac{2(n-1)^{\frac{3}{2}}}{3 n-2} \leq 1.4
$$

The effect of the fading distribution on the Gaussianity of $I_{A}$ is discussed in Section 6.3.

\subsubsection{No Exclusion Region $\left(r_{o}=0\right)$}

If there is no exclusion region around the victim receiver, i.e., $r_{o}=0$, then it can be shown from (6.3) and (6.4) that the Berry-Esseen bound becomes

$$
\left|F_{Z}(y)-F_{N}(y)\right| \leq 2.21 \frac{3(n-1)^{\frac{3}{2}}}{\sqrt{n}(3 n-2)} \frac{1}{\sqrt{\lambda \pi r_{c}^{2}}} \frac{\tilde{\mu}_{3}(X)}{\left[\tilde{\mu}_{2}(X)\right]^{\frac{3}{2}}}
$$


Remarks:

- From (6.7), it might be concluded that a high $r_{c}$ value improves the Gaussian approximation. However, $r_{c}$ is used in the path-loss model to avoid the singularity at $r_{i}=0$. The model effectively fixes the distance-dependant attenuation over a small disk of radius $r_{c}$ around the victim receiver. Therefore, the value of $r_{c}$ should be kept relatively small.

- To justify the Gaussian approximation for this topology, $\sqrt{\lambda \pi r_{c}^{2}}$ should be large. However, since $r_{c}$ is relatively small then $\lambda$ should be very high. To demonstrate this, let us assume that there are two networks: $\operatorname{Net}_{\left(r_{o}=100\right)}$ with an exclusion region of $r_{o}=100$ meters and $\operatorname{Net}_{\left(r_{o}=0\right)}$ without an exclusion region. Except for $r_{o}$, these two networks are assumed to have the same system and channel parameters including $r_{c}=1$ meter and $n=3$. Assuming that these two networks have the same active node densities, the Berry-Esseen bound for $\operatorname{Net}_{\left(r_{o}=100\right)}$ can be shown to be smaller than the one for $\operatorname{Net}_{\left(r_{o}=0\right)}$ by about $20 \mathrm{~dB}$. Therefore, the distribution of $I_{A}$ in $\operatorname{Net}_{\left(r_{o}=100\right)}$ is much closer to Gaussianity in comparison to the distribution of $I_{A}$ in $\operatorname{Net}_{\left(r_{o}=0\right)}$. For $\operatorname{Net}_{\left(r_{o}=0\right)}$ to have the same BerryEsseen bound value as of $\operatorname{Net}_{\left(r_{o}=100\right)}$, the active node density in $\operatorname{Net}_{\left(r_{o}=0\right)}$ has to be about $40 \mathrm{~dB}$ higher, which might be too high unless the active nodes density of $\operatorname{Net}_{\left(r_{o}=100\right)}$ is too low.

- Therefore, the Gaussian approximation could be possible for wireless networks with a sufficiently wide exclusion region. However, the validity of the approximation is questionable when there is no exclusion region or when the exclusion region is small ${ }^{5}$, unless the active node density is very high which might be practically infeasible.

\footnotetext{
${ }^{5}$ The authors in [48] shows that a lognormal distribution can be used to approximate the distribution of the aggregate interference power when the exclusion region is small.
} 
- From (6.5) and (6.7), it might be deduced that the Gaussian approximation is valid if

$$
\sqrt{\lambda \pi r_{o}^{2}}>>2.21 \frac{2(n-1)^{\frac{3}{2}}}{3 n-2} \frac{\tilde{\mu}_{3}(X)}{\left[\tilde{\mu}_{2}(X)\right]^{\frac{3}{2}}}, \text { for } r_{o} \geq r_{c}
$$

or

$$
\sqrt{\lambda \pi r_{c}^{2}}>>2.21 \frac{3(n-1)^{\frac{3}{2}}}{\sqrt{n}(3 n-2)} \frac{\tilde{\mu}_{3}(X)}{\left[\tilde{\mu}_{2}(X)\right]^{\frac{3}{2}}}, \text { for } r_{o}=0 .
$$

\subsection{Effect of Fading Distributions on the Gaussian Approxi- mation of $I_{A}$}

In this section, we investigate the effect of the distribution of $X_{i}$, i.e., the fading distribution, on the Gaussian convergence of the distribution of $I_{A}$. We provide expressions for $\tilde{\mu}_{3}(X) /\left[\tilde{\mu}_{2}(X)\right]^{\frac{3}{2}}$ considering different fading cases. These expressions can be used with (6.4), (6.5) or (6.7) to get the related Berry-Esseen bounds.

\subsubsection{Case I: Without Multipath Fading and without Shadow Fading}

For the case without fading (neither multipath nor shadow fading), $X_{i}$ becomes deterministic. Therefore,

$$
\frac{\tilde{\mu}_{3}(X)}{\left[\tilde{\mu}_{2}(X)\right]^{\frac{3}{2}}}=1
$$

which leads to

$$
\left|F_{Z}(y)-F_{N}(y)\right|<2.21 \frac{2(n-1)^{\frac{3}{2}}}{3 n-2} \frac{1}{\sqrt{\lambda \pi r_{o}^{2}}} .
$$

Equation (6.11) may be considered as the baseline to judge the effect of fading on the Gaussian convergence. 


\subsubsection{Case II: With Multipath Fading but without Shadow Fading}

The effect of multipath fading on the received individual interference power $I_{i}$ can be modeled by a Gamma random variable (which is a result of the assumption that the envelope of the interference signal can be modeled by the versatile Nakagami distribution). In this case, the probability density function (PDF) of $X_{i}$ can be represented as [93]

$$
f_{X}(x)=\left(\frac{\nu}{\Omega}\right)^{\nu} \frac{x^{\nu-1}}{\Gamma(\nu)} e^{-\frac{\nu}{\Omega} x}, x>0, \nu \geq \frac{1}{2}
$$

where $\Omega$ is the average received power, $\nu$ is the shape parameter, and $\Gamma(\cdot)$ is the Gamma function. The $m$ th moment of $X_{i}$ can be expressed as

$$
\tilde{\mu}_{m}(X)=\left(\frac{\Omega}{\nu}\right)^{m} \frac{\Gamma(\nu+m)}{\Gamma(\nu)} .
$$

Thus,

$$
\frac{\tilde{\mu}_{3}(X)}{\left[\tilde{\mu}_{2}(X)\right]^{\frac{3}{2}}}=\frac{\Gamma(\nu+3)}{[\Gamma(\nu+2)]^{\frac{3}{2}}} \Gamma(\nu)^{\frac{1}{2}}=\frac{\nu+2}{\sqrt{\nu(\nu+1)}} .
$$

We may conclude that the multipath fading shifts the distribution of the $I_{A}$ away from Gaussianity. However, the shift is limited since, for $\frac{1}{2} \leq \nu<\infty$,

$$
1<\frac{\nu+2}{\sqrt{\nu(\nu+1)}} \leq 2.89
$$

\subsubsection{Case III: With Shadow Fading but without Multipath Fading}

The shadow fading is commonly modeled by a lognormal random variable with logarithmic mean $0 \mathrm{~dB}$ and standard deviation $\sigma_{s} \mathrm{~dB}$. Therefore, the effect of shadow fading on the aggregate interference power can be reflected by assuming that $X_{i}$ is a lognormal random variable, and hence it has the following expression for its $m$ th 


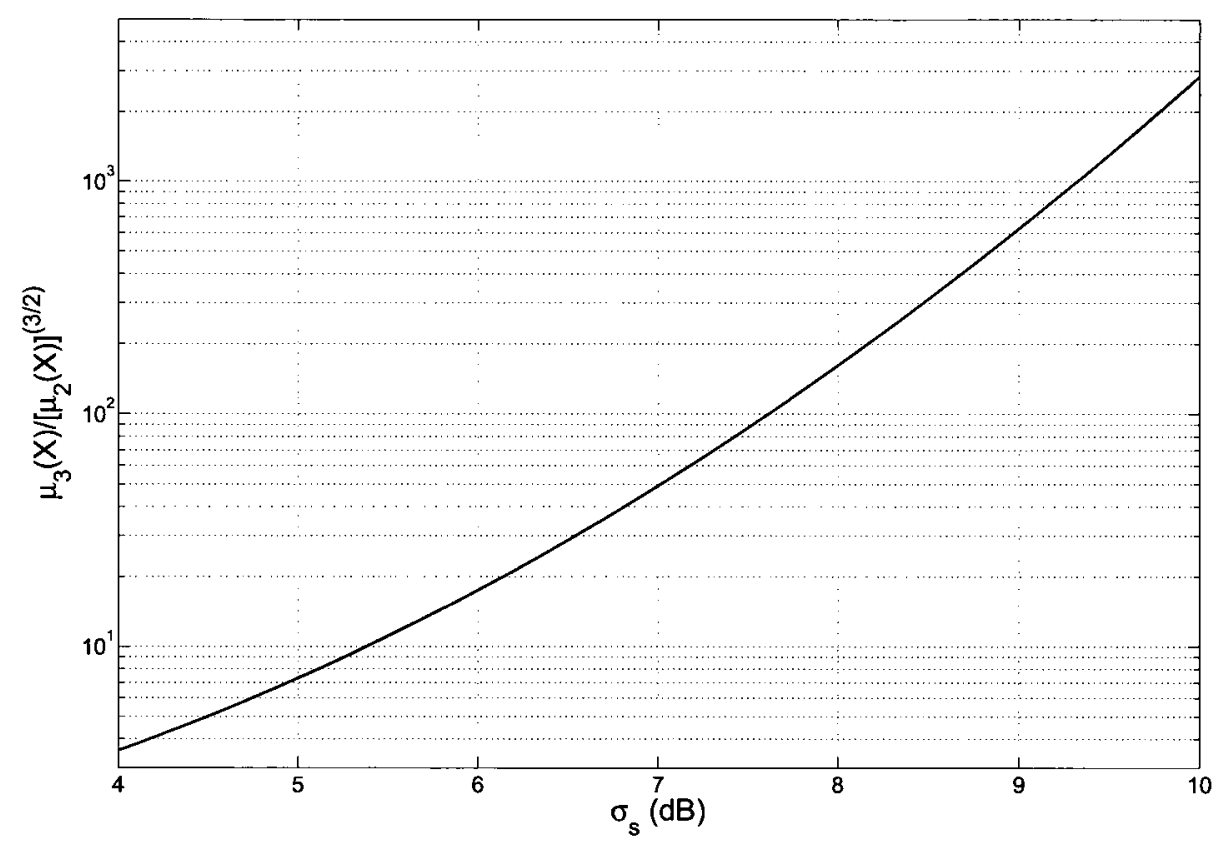

Figure 6.4: Values of $\tilde{\mu}_{3}(X) /\left[\tilde{\mu}_{2}(X)\right]^{\frac{3}{2}}$ for different values of $\sigma_{s}$.

moment:

$$
\tilde{\mu}_{m}(X)=e^{\frac{1}{2}\left(m \frac{\ln 10}{10} \sigma_{s}\right)^{2}}
$$

which leads to

$$
\frac{\tilde{\mu}_{3}(X)}{\left[\tilde{\mu}_{2}(X)\right]^{\frac{3}{2}}}=e^{\frac{3}{2}\left(\frac{\ln 10}{10} \sigma_{s}\right)^{2}} .
$$

As shown in Fig. 6.4, for typical values of $\sigma_{s}$, e.g., $\sigma_{s} \in[4,10] \mathrm{dB}$, the effect of shadow fading on $\tilde{\mu}_{3}(X) /\left[\tilde{\mu}_{2}(X)\right]^{3 / 2}$, and consequently on the Berry-Esseen bound, could be dominant compared to the effect of multipath fading.

\subsubsection{Case IV: With Composite Fading}

If the interference signals are subject to both multipath and shadow fading, $X_{i}$ can be modeled as the product of two independent random variables, such as a Gamma random variable (for the multipath fading) and a lognormal random variable (for the 
shadow fading). Thus, using (6.14) and (6.17),

$$
\frac{\tilde{\mu}_{3}(X)}{\left[\tilde{\mu}_{2}(X)\right]^{\frac{3}{2}}}=\frac{\nu+2}{\sqrt{\nu(\nu+1)}} e^{\frac{3}{2}\left(\frac{\ln 10}{10} \sigma_{s}\right)^{2}}
$$

\subsubsection{General Remarks}

Having multipath or shadow fading shifts the distribution of $I_{A}$ away from Gaussianity. To maintain the Gaussianity, the density of active nodes should be increased or the exclusion region should be extended.

Fig. 6.5 reflects the divergence from the Gaussian distribution that the fading distribution may cause. It shows three different fading scenarios. It is clear from the figure that the divergence caused by lognormal shadow fading with $\sigma_{s}=6 \mathrm{~dB}$ is more than the one caused by the Rayleigh multipath fading. In the presence of significant shadow fading, the Gaussian distribution is a very poor approximation for the distribution of $I_{A}$ unless the active node density is too high or the exclusion region is too wide.

\subsection{Conclusions}

In this chapter, we studied the convergence of this distribution to a Gaussian distribution. Based on the Berry-Esseen bound, we casted in a single mathematical framework some observations scattered across the literature about the Gaussianity of the distribution of the aggregate interference power. We showed that an increase in the size of the exclusion region brings the distribution of the aggregate interference power closer to the Gaussian distribution. Increasing the active node density has a similar effect. However, the convergence is faster with the increase in the size of the exclusion region compared to the increase in the active node density. In contrast, channel fading causes divergence from Gaussianity. Shadow fading typically causes 


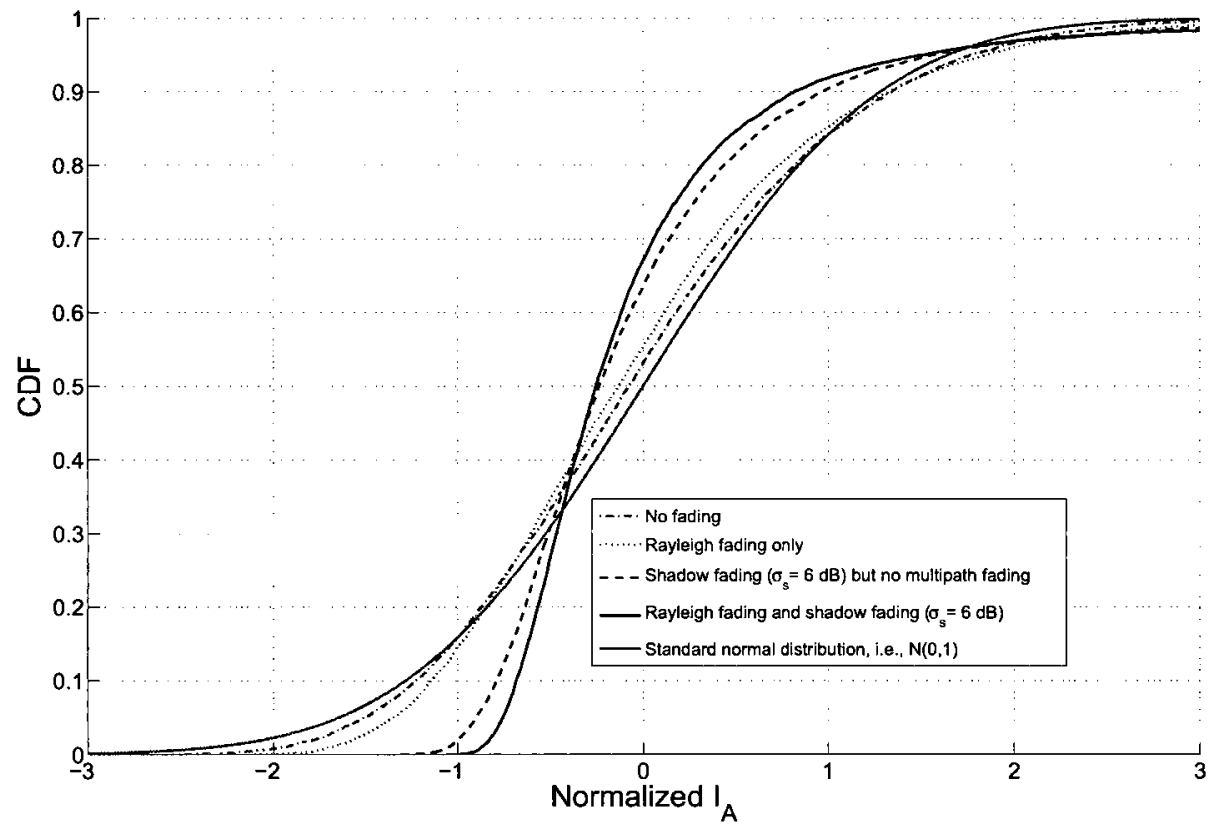

Figure 6.5: Monte-Carlo simulation-based CDF for the normalized $I_{A}$, i.e., $\frac{I_{A}-\tilde{\mu}_{A}}{\sigma_{A}}$, for different fading scenarios $\left(r_{o}=10\right.$ meters, $r_{c}=1$ meter, $n=3$, and $\lambda={ }_{0}^{\sigma_{A}} .01$ nodes $/$ meter $\left.^{2}\right)$.

more divergence as compared to multipath fading. 


\section{Chapter 7}

\section{Identifying Boundaries of Dominant Regions Dictating Spectrum Sharing Opportunities}

It is indicated in previous chapters and in the literature as well that the aggregate interference power of an infinite network (such as a very large secondary network) is bounded under certain conditions. Moreover, we have shown in Chapter 5.6 that the $m$ th cumulant tends to a constant as $L$ goes to infinity (or practically when $L \gg r_{o}$ ). A similar tendency behavior has been observed for the interference probability as well. However, Chapter 5.6 does not discuss the boundary of the region that dominantly sets the values of cumulants, interference probability, and hence the spectrum sharing opportunities. Therefore, the objective of this chapter is to identify the smallest region of the secondary network that would dominantly impact spectrum sharing opportunities. Our results show that the dominant region is not necessarily a small region encompassing a few interferers within the proximity of the primary user. Far interferers may tangibly contribute to spectrum sharing decisions when a higher approximation accuracy is required or when a wide exclusion region is considered. On the other hand, the dominant region shrinks with increase in the path-loss exponent or in the level of the interference threshold specified by the primary user or a regulator. Some implications of these results are highlighted in this chapter. Moreover, the 
results are anticipated to inspire new ideas for designing MAC protocols for secondary networks.

\subsection{Introduction}

It is indicated in Chapter 5.6, [53], [57] and some of the references therein that the interference of an infinite wireless network is bounded when the path-loss exponent is strictly greater than the dimension of the space of the network. We show in Chapter 5.6 that the cumulants of the aggregate interference asymptotically approach constant values as the spatial size of the network increases provided that the density of interfering nodes remains constant. While there are some comments in the literature (e.g., in [19] and [20]) indicating that the aggregate interference is dominated by the nearby interferers to the victim receiver, there is to the best of our knowledge no work devoted to identifying the boundary of the dominant region. Our contribution comes to fill this gap, especially in the context of spectrum sharing. Results reported in this chapter are anticipated to be useful for designing MAC protocols for large wireless secondary networks to create and maintain spectrum sharing opportunities.

To simplify the flow of discussions in this chapter, the next section provides a brief summary of relevant results obtained in previous chapters for cumulants and approximations of the aggregate interference power. Then, Section IV provides analysis and discussions on the dominant region. Finally, the chapter is concluded with some remarks in Section 7.4.

\subsection{Cumulants of $I_{A}$ and the Interference Probability}

As mentioned before, a viable approach to characterize the distribution of $I_{A}$ is to use the characteristic function to calculate cumulants of $I_{A}$. These cumulants are then used to approximate the distribution of $I_{A}$. Applying cumulants expressions obtained 


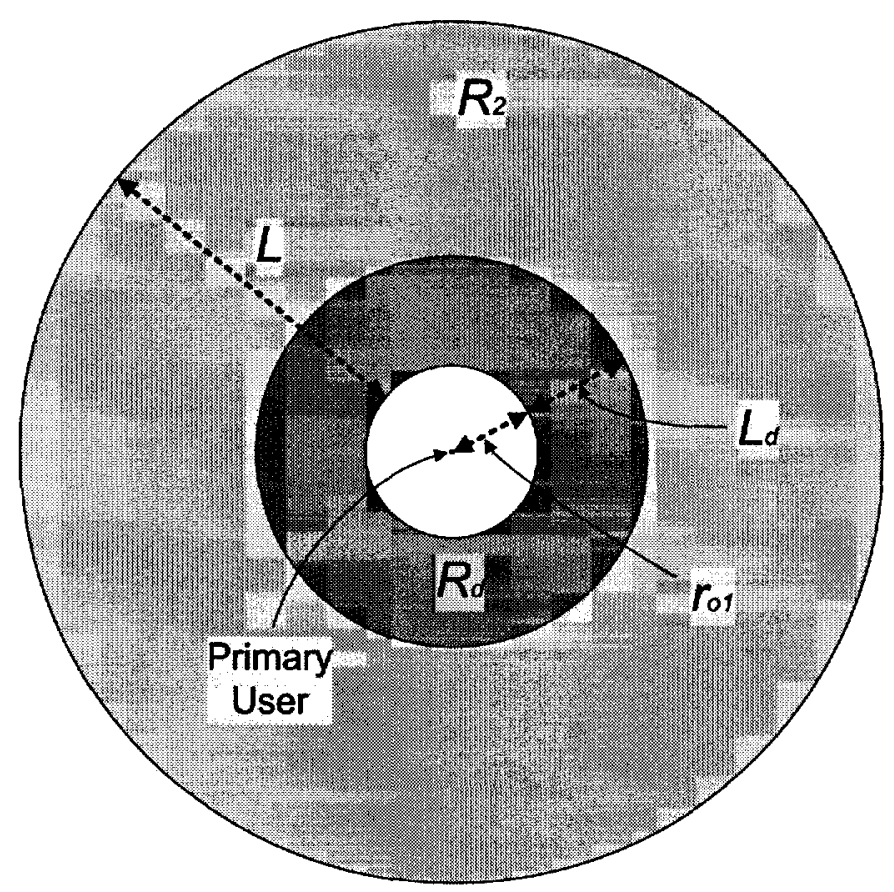

Figure 7.1: Layout of a partitioned secondary network. In this chapter, we consider $L \rightarrow \infty$. The dominant region $\mathcal{R}_{d}$ has an inner radius of $r_{o}$ and an outer radius $r_{o}+L_{d}$. The inner white circle is the exclusion region. The region $\mathcal{R}_{t}=\mathcal{R}_{d} \cup \mathcal{R}_{2}$ corresponds to the whole secondary network.

in Chapter 5 for the network shown in Fig. 7.1 leads to

$$
\begin{aligned}
& \kappa_{m}\left(I_{A}\right)=\lambda \pi \tilde{\mu}_{m}(X)\left[g\left(r_{o}\right)\right]^{m} \\
& \times\left[\hat{r}^{2}\left(1+\frac{2}{m n-2}\left(1-\left[\frac{\hat{r}}{r_{o}+L}\right]^{m n-2}\right)\right)-r_{o}^{2}\right],
\end{aligned}
$$

where $\hat{r}=\max \left(\min \left(r_{c}, r_{o}+L\right), r_{o}\right)$, and $\tilde{\mu}_{m}(X)$ denotes the $m$ th raw moment of $X_{i}$ (the subscript $i$ is omitted for convenience and since $X_{i} \mathrm{~s}$ are i.i.d.). In this chapter, we consider an infinite field, i.e., $L \rightarrow \infty$.

These cumulants could be utilized to approximate the distribution of the aggregate interference power. A good approximating distribution that could be used for this purpose is the shifted lognormal distribution $[12,22]$. The probability distribution function of the shifted lognormal random variable can be expressed as 


$$
f_{Z}(z)=\frac{1}{s(z-b) \sqrt{2 \pi}} e^{-(\ln (z-b)-u)^{2} / 2 s^{2}}, z>b
$$

where $s, b$, and $u$ can be calculated using the cumulants of $I_{A}$ according to the following equations:

$$
\begin{aligned}
s^{2} & =\ln \tau \\
u & =\frac{1}{2} \ln \frac{\kappa_{2}\left(I_{A}\right)}{\tau(\tau-1)}, \\
b & =\kappa_{1}\left(I_{A}\right)-\sqrt{\frac{\kappa_{2}\left(I_{A}\right)}{\tau-1}} \\
\tau & =\left[v+\sqrt{v^{2}-1}\right]^{1 / 3}+\left[v-\sqrt{v^{2}-1}\right]^{1 / 3}-1, \\
v & =1+\frac{1}{2} \rho^{2} \\
\rho & =\frac{\kappa_{3}\left(I_{A}\right)}{\left[\kappa_{2}\left(I_{A}\right)\right]^{3 / 2}} .
\end{aligned}
$$

Based on this approximation, the interference probability of $I_{A}$ can be expressed as

$$
P\left(I_{A} \geq I_{t h}\right) \simeq Q\left(\frac{\ln \left(I_{t h}-b\right)-u}{s}\right)
$$

\subsection{The Dominant Region}

As mentioned before, the objective of this chapter is to determine the boundary of the dominant region. Therefore, we present in this section two different approaches to identifying the dominant interfering region. There might be other approaches, but these two are deemed to be sufficient to explain the concept. The first approach is based on the cumulants while the other one is based on the interference probability. In this chapter, we focus on the case where there is an exclusion region around the $\mathrm{PU}$ with $r_{o} \geq r_{c}$. Other cases can be investigated in a similar way by substituting $\hat{r}$ and $g\left(r_{o}\right)$ in $(7.1)$ with their corresponding expressions. 


\subsubsection{A Cumulant-Based Approach}

Let us define the dominant region for the $m$ th cumulant to be the minimum region around the PU-RX that approximately produces the same $m$ th cumulant as the one obtained for the whole network. To quantify this approximation, we assume that the PU-RX is insensitive to, or can tolerate, a maximum relative approximation error $\epsilon_{\kappa}$ in the calculation of cumulants. Denoting the $m$ th cumulant of the interference from the whole network as $\kappa_{m}\left(I_{A}, \mathcal{R}_{t}\right)$, and $m$ th cumulant of the interference from the dominant region only as $\kappa_{m}\left(I_{A}, \mathcal{R}_{d}\right)$, the approximation error can be expressed as

$$
\frac{\kappa_{m}\left(I_{A}, \mathcal{R}_{t}\right)-\kappa_{m}\left(I_{A}, \mathcal{R}_{d}\right)}{\kappa_{m}\left(I_{A}, \mathcal{R}_{t}\right)} \leq \epsilon_{\kappa}
$$

where $\epsilon_{\kappa}$ denotes the maximum acceptable error in approximating $\kappa_{m}\left(I_{A}, \mathcal{R}_{t}\right)$ by $\kappa_{m}\left(I_{A}, \mathcal{R}_{d}\right)$. The error should be very small, i.e., $\epsilon_{\kappa} \ll 1$. The expression (7.5) is established based on one of the common approximation error expressions known as a relative error expression [79]. The difference is divided by $\kappa_{m}\left(I_{A}, \mathcal{R}_{t}\right)$ for normalization. The expressions for $\kappa_{m}\left(I_{A}, \mathcal{R}_{t}\right)$ and $\kappa_{m}\left(I_{A}, \mathcal{R}_{d}\right)$ are obtained from (7.1) by letting $L \rightarrow \infty$ and $L=L_{d}$, respectively. By inserting the expressions of $\kappa_{m}\left(I_{A}, \mathcal{R}_{t}\right)$ and $\kappa_{m}\left(I_{A}, \mathcal{R}_{d}\right)$ into (7.5) and performing some mathematical manipulations, we get

$$
\left(\frac{r_{o}}{r_{o}+L_{d}}\right)^{m n-2} \leq \epsilon_{\kappa}
$$

which leads to

$$
L_{d} \geq\left(\epsilon_{\kappa}^{\frac{1}{2-m n}}-1\right) r_{o} .
$$

The dominant region can be defined as the minimum $L_{d}$ that satisfies the inequality in (7.7). From (7.7), it is observed that the dominant region is a function of the cumulant order, i.e., $m$. As $m$ increases, the dominant region shrinks. The dominant 
region for the mean (i.e., $\kappa_{1}$ ) is bigger than the one for the variance, i.e., $\kappa_{2}$. Therefore, if a region provides a satisfactory approximation for the mean of $I_{A}$, then it also provides satisfactory approximations for higher cumulants. It is also clear from (7.7) that a wider exclusion region $\left(r_{o}\right)$ leads to a larger value of $L_{d}$. Moreover, (7.7) indicates that the higher the path-loss exponent is, the smaller the dominant region becomes.

As an example, if $r_{o}=1 \mathrm{~m}$ and $n=3$, approximating the whole network by a region of $L_{d}=10 \mathrm{~m}$ generates a maximum approximation error of 0.091 for the mean. The same region, i.e., $L_{d}=10 \mathrm{~m}$, provides a smaller approximation error of $10^{-4}$ for the variance. Based on (7.7), Table 7.1 shows the values of $L_{d}$ calculated for different values of maximum approximation errors in the first three cumulants. The table also reflects the effect of the exclusion region and the path-loss exponent on $L_{d}$.

It is worth noting that the dominant region from the cumulants' perspective does not depend on transmit power, antenna gains, or fading distributions. The results on the dominant region from the perspective of cumulants give us some insight on the dynamics of the aggregate interference. The results also give some hints on what portion of the secondary network would dictate the spectrum sharing opportunities. However, a solid investigation of the dominant region of the secondary network from the perspective of spectrum sharing opportunities should be based on the interference probability or on some other appropriate harmful interference metrics.

\subsubsection{An Interference Probability-Based Approach}

From the perspective of spectrum sharing, the dominant region could be defined as the minimum portion of a secondary network that would provide approximately the same spectrum sharing conclusions as those drawn by considering the whole secondary network. In this chapter, we use the interference probability as the metric for the spectrum sharing opportunity. This interference probability is mainly based on the 
Table 7.1: $L_{d}$ calculated for different values of maximum approximation errors in the first three cumulants. Calculations are repeated for different values of $r_{o}$ and $n$ to reflect their effects.

\begin{tabular}{c|c|c||c|c|c}
\hline$r_{o}$ & $n$ & $\epsilon_{\kappa}$ & $L_{d}$ for $\kappa_{1}$ & $L_{d}$ for $\kappa_{2}$ & $L_{d}$ for $\kappa_{3}$ \\
\hline \hline & & 0.1 & $9 \mathrm{~m}$ & $0.78 \mathrm{~m}$ & $0.39 \mathrm{~m}$ \\
$1 \mathrm{~m}$ & 3 & 0.01 & $99 \mathrm{~m}$ & $2.16 \mathrm{~m}$ & $0.93 \mathrm{~m}$ \\
& & 0.001 & $999 \mathrm{~m}$ & $4.62 \mathrm{~m}$ & $1.68 \mathrm{~m}$ \\
\hline & & 0.1 & $90 \mathrm{~m}$ & $7.78 \mathrm{~m}$ & $3.89 \mathrm{~m}$ \\
$10 \mathrm{~m}$ & \multirow{3}{*}{3} & 0.01 & $990 \mathrm{~m}$ & $21.62 \mathrm{~m}$ & $9.31 \mathrm{~m}$ \\
& & 0.001 & $9990 \mathrm{~m}$ & $46.23 \mathrm{~m}$ & $16.83 \mathrm{~m}$ \\
\hline & & 0.1 & $21.62 \mathrm{~m}$ & $4.68 \mathrm{~m}$ & $2.59 \mathrm{~m}$ \\
$10 \mathrm{~m}$ & \multirow{2}{*}{4} & 0.01 & $90.00 \mathrm{~m}$ & $11.54 \mathrm{~m}$ & $5.85 \mathrm{~m}$ \\
& & 0.001 & $306.23 \mathrm{~m}$ & $21.62 \mathrm{~m}$ & $9.95 \mathrm{~m}$ \\
\hline
\end{tabular}

CCDF of $I_{A}$. However, Fig. 7.2 and 7.3 demonstrate that the CCDF of $I_{A}$ converges to a limiting distribution as $L$ increases. The convergence occurs faster for smaller $r_{o}$. Therefore, there is a dominant region that would satisfy the following condition:

$$
\frac{P_{\text {int }}\left(I_{\text {th }}, \mathcal{R}_{t}\right)-P_{\text {int }}\left(I_{\text {th }}, R_{d}\right)}{P_{\text {int }}\left(I_{\text {th }}, \mathcal{R}_{t}\right)} \leq \epsilon
$$

where $P_{\text {int }}\left(I_{A}, \mathcal{R}_{t}\right)$ is the interference probability considering the aggregate interference from the whole field and a threshold level of $I_{t h} . P_{\text {int }}\left(I_{t h}, \mathcal{R}_{d}\right)$ is the interference probability considering the interference coming from $\mathcal{R}_{d}$ only with the same $I_{t h}$. The parameter $\epsilon$ denotes the maximum acceptable error in approximating $P_{i n t}\left(I_{A}, \mathcal{R}_{t}\right)$ by $P_{\text {int }}\left(I_{\text {th }}, \mathcal{R}_{d}\right)$.

Assuming that the aggregate interference power of $\mathcal{R}_{t}$, and the one of $\mathcal{R}_{d}$ are approximated by shifted lognormal random variables, (7.8) can be expressed as

$$
1-\frac{Q\left(\frac{\ln \left(I_{t h}-b\left(L_{d}\right)\right)-u\left(L_{d}\right)}{s\left(L_{d}\right)}\right)}{Q\left(\frac{\ln \left(I_{t h}-b\right)-u}{s}\right)} \leq \epsilon
$$




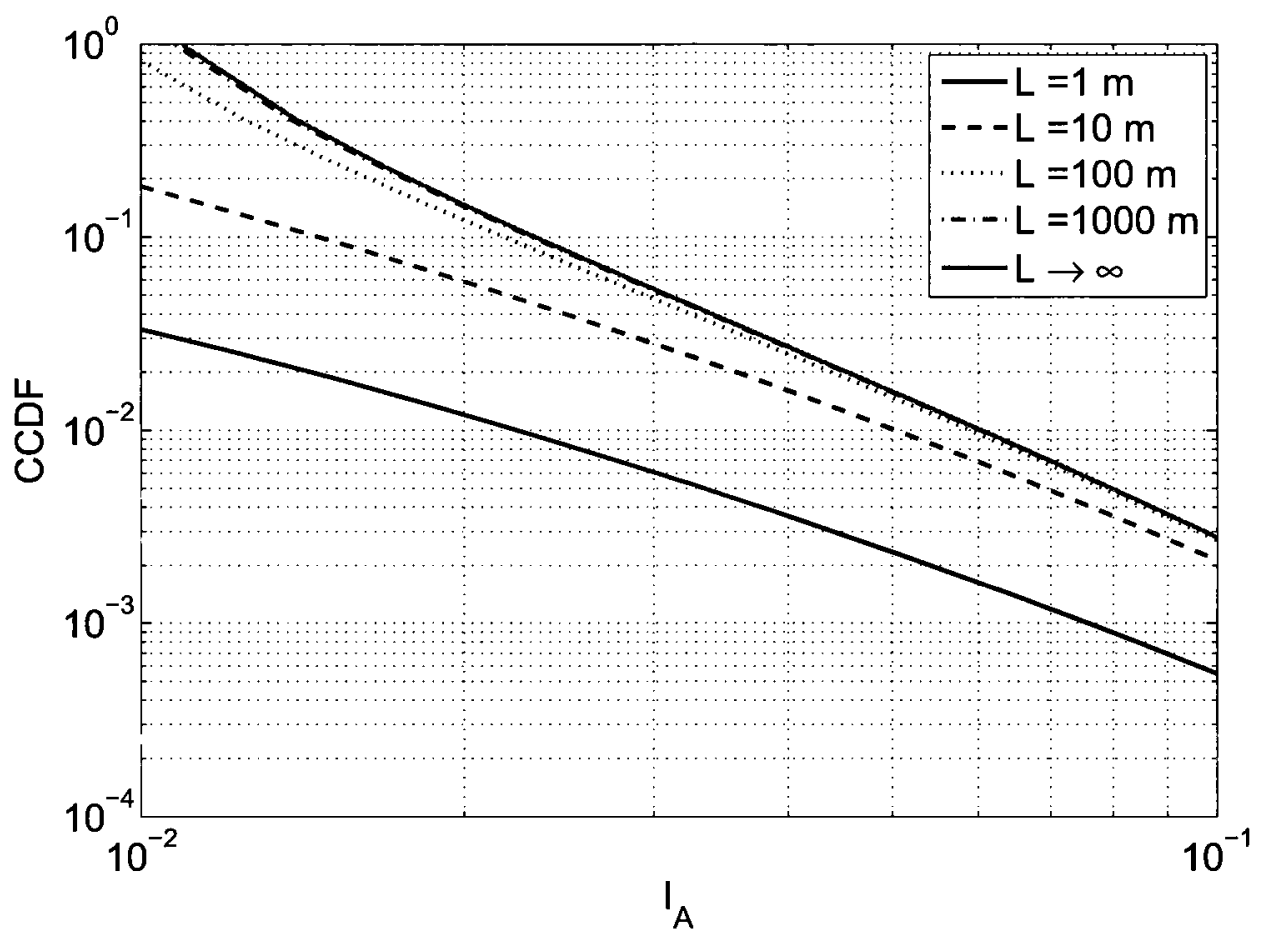

Figure 7.2: The CCDF of the aggregate interference power based on a shifted lognormal approximation for different values of $L\left(r_{o}=10 \mathrm{~m}, r_{c}=1 \mathrm{~m}, \lambda=0.01\right.$ nodes $/ \mathrm{m}^{2}$, $n=3$, Rayleigh fading, and $6 \mathrm{~dB}$ shadowing).

We use the notations $b\left(L_{d}\right), u\left(L_{d}\right)$, and $s\left(L_{d}\right)$ to indicate that these parameters are obtained from the cumulants of the interference power $I_{A}$ coming from $\mathcal{R}_{d}$. To identify the boundary of the dominant region, (7.9) should be solved for $L_{d}$ with the equality. This dominant region should be sufficient to investigate the spectrum sharing opportunities for the whole secondary networks. To demonstrate this, let us assume that the $\mathrm{PU}$ or the regulator specifies that the spectrum of the $\mathrm{PU}$ can be shared by the secondary network if $P\left(I_{A} \geq I_{t h}\right) \leq 0.1$, where $I_{t h}=0.0233$ (a normalized value with respect to the transmit power, antenna gains and other constant parameters). If the $\mathrm{PU}$ is insensitive to, or can tolerate an error in the interference probability of $1 \%$ or less (i.e., $\epsilon \leq 0.01$ ), then according to Fig. 7.4 the minimum $L_{d}$ satisfying $\epsilon \leq 0.01$ is $1700 \mathrm{~m}$ which defines the boundary of the corresponding dominant region. Fig. 7.4 is plotted based on (7.9) for system and channel parameters 


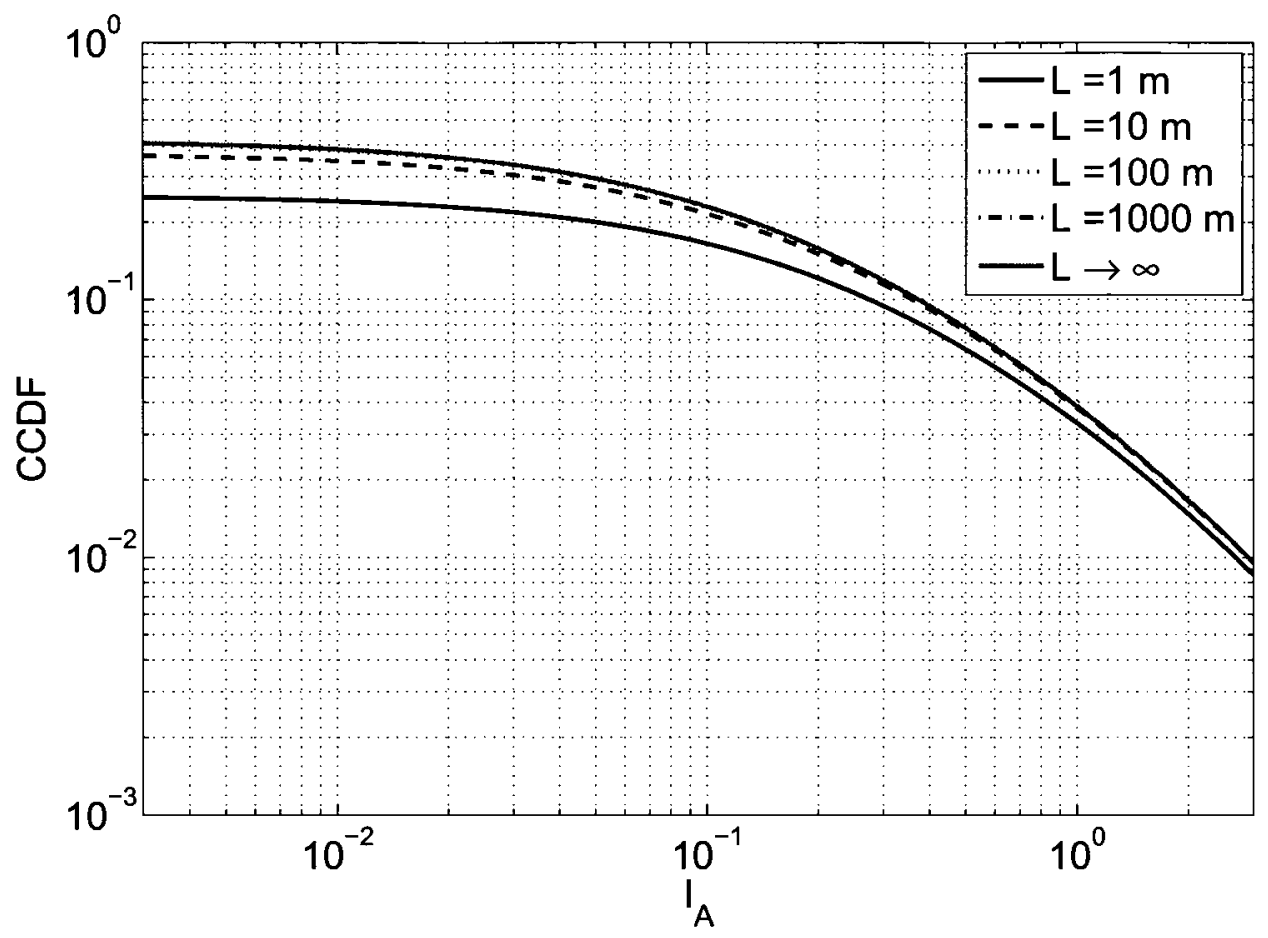

Figure 7.3: The CCDF of the aggregate interference power based on a shifted lognormal approximation for different values of $L\left(r_{o}=1 \mathrm{~m}, r_{c}=1 \mathrm{~m}, \lambda=0.01\right.$ nodes $/ \mathrm{m}^{2}$, $n=3$, Rayleigh fading, and $6 \mathrm{~dB}$ shadowing).

indicated in the caption of the figure including an exclusion region of $10 \mathrm{~m}$. If the PU can tolerate a larger error, e.g., $\epsilon \leq 0.1$, then $L_{d}=150 \mathrm{~m}$ for the same $I_{t h}$ and the same system and channel parameters. If $I_{t h}$ is specified at a higher value, e.g., 0.0604 , then $L_{d}$ decreases to $650 \mathrm{~m}$ and $50 \mathrm{~m}$ for $\epsilon=0.01$ and 0.1 , respectively.

Before investigating the effect of $r_{o}$ on the dominant region by comparing Fig. 7.4 and 7.5 , it is worth noting that we choose the values of $I_{t h}$ in these figures to have identical interference probabilities. From Fig. 7.5 and for $I_{t h}=0.374$ (corresponding to $\left.P_{\text {int }}\left(I_{t h}, \mathcal{R}_{t}\right)=0.1\right), L_{d}=34.6 \mathrm{~m}$ for $\epsilon=0.01$ and $2.44 \mathrm{~m}$ for $\epsilon=0.1$. These values of $L_{d}$ are smaller than those obtained from Fig. 7.4 when $r_{o}=10 \mathrm{~m}$ and $I_{t h}=.0233$, which corresponds to $P_{\text {int }}\left(I_{t h}, \mathcal{R}_{t}\right)=0.1$.

In summary, the dominant region decreases as $\epsilon$ increases. On the other hand, it 


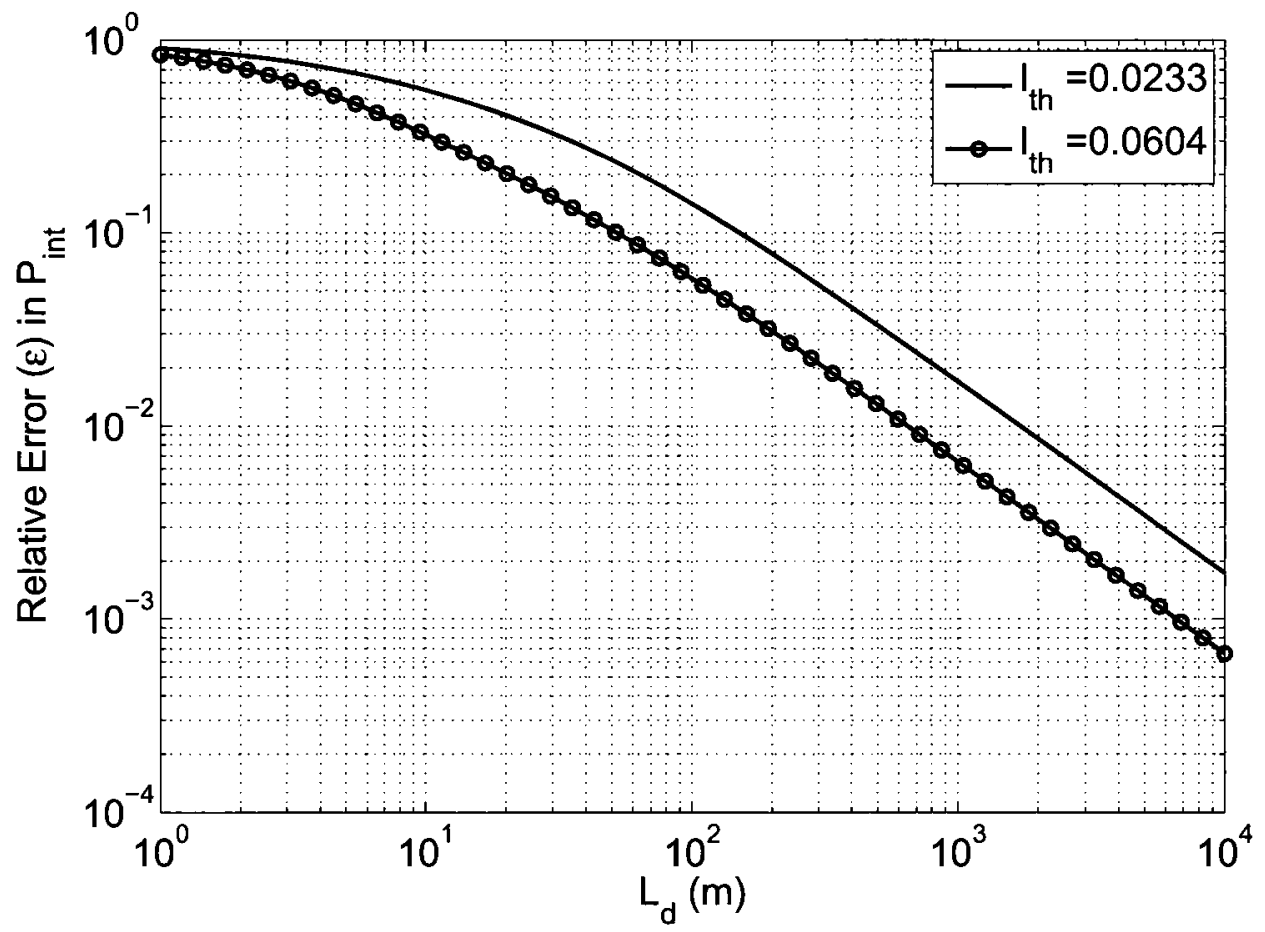

Figure 7.4: Relative approximation error in the interference probability for different values of $I_{t h}\left(r_{o}=10 \mathrm{~m}, r_{c}=1 \mathrm{~m}, \lambda=0.01\right.$ nodes $/ \mathrm{m}^{2}, n=3$, Rayleigh fading, and $6 \mathrm{~dB}$ shadowing). The values of $I_{t h}$ are chosen to correspond to the interference probability of 0.1 and 0.01 , respectively, that a PU-RX would experience from an infinite secondary network.

increases as $r_{o}$ increases. These observations are similar to the findings on the dominant region from the perspective of cumulants in the previous subsection. However, the dominant region from the perspective of the interference probability also depends on the value of $I_{t h}$, which does not appear in the formulations of the cumulant-based dominant region.

\subsubsection{Implications of the Dominant Region}

Following are some implications of the results obtained for the dominant region.

- In interference limited systems, there might be a tendency to assume that performance measures of the system are affected by a few interferers around a 


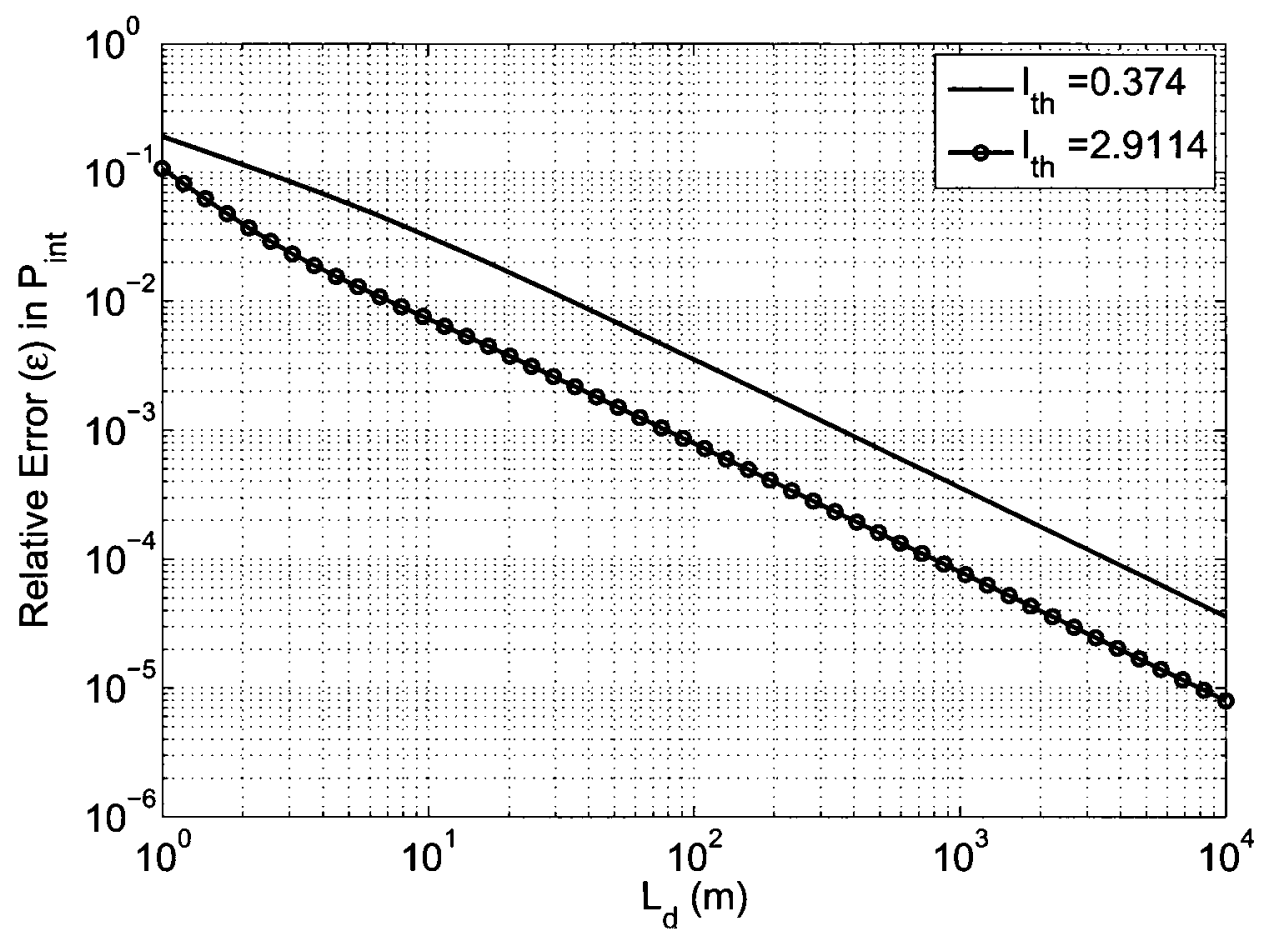

Figure 7.5: Relative approximation error in the interference probability for different values of $I_{t h}\left(r_{o}=1 \mathrm{~m}, r_{c}=1 \mathrm{~m}, \lambda=0.01\right.$ nodes $/ \mathrm{m}^{2}, n=3$, Rayleigh fading, and $6 \mathrm{~dB}$ shadowing). The values of $I_{t h}$ are chosen to correspond to the interference probability of 0.1 and 0.01 , respectively, that a PU-RX would experience from an infinite secondary network.

victim receiver. While this assumption could be valid for many scenarios, it might be invalid for other scenarios. Therefore, it is advisable to verify this assumption by identifying the dominant region around the victim receiver.

- Simulations of the interference and spectrum sharing opportunities in large networks can be significantly simplified, without degrading the accuracy, by simulating the dominant region only not the whole network.

- A PU-RX who is within a finite secondary network but away from the edge of the network by a distance of $L_{d}$ or more is practically receiving the same level of interference as if it is located at the center of the secondary network. Hence, a PU-RX has almost identical influence on spectrum sharing decisions regardless 
of its location within the secondary network as long as it is away from the edge by a minimum distance of $L_{d}$.

- Any deployments of SU-TXs outside the dominant region has no effect on the spectrum sharing decisions provided that the density of SU-TXs outside the dominant region does not exceed the density of the SU-TXs within the dominant region.

- We anticipate that our results in this chapter would inspire new ideas for designing MAC protocols for secondary networks.

\subsection{Conclusions}

In this chapter, we investigated the dominant region that dictates the spectrum sharing opportunities of a large secondary network. We identified the boundary of this dominant region. We showed that as the exclusion region increases the dominant region increases as well. However, the dominant region shrinks with the increase in the path-loss exponent. The higher the required accuracy is, the wider the dominant region becomes. In addition, we demonstrated that the boundary of the dominant region shrinks with the increase of the interference threshold level. Results reported in this chapter are anticipated to be useful for designing MAC protocols and algorithms for large wireless secondary networks to create and maintain spectrum sharing opportunities. 


\section{Chapter 8}

\section{Conclusions and Suggested Future Work}

\subsection{Summary}

In this thesis, we addressed the interference characterization and spectrum sharing in large secondary networks. We started by characterizing the distribution of the aggregate interference power based on its cumulants. We utilized these cumulants to approximate the distribution of the aggregate interference power. We also investigated the convergence of this distribution to a Gaussian distribution. Based on results obtained for the interference characterization, we investigated the spectrum sharing in large networks including the effect of the spatial size of the secondary network on spectrum sharing opportunities. We then addressed the dominant interfering region in large networks with the objective to draw a boundary for this region. In our analysis, we rely on techniques borrowed from stochastic geometry especially the PPP that we used to model the spatial distribution of active secondary users. Moreover, we used the interference probability as the harmful interference metric to map the level of the aggregate interference power to appropriate spectrum sharing decisions. 


\subsection{Contributions}

The following subsections highlight the major contributions of the work presented in this thesis.

\subsubsection{Cumulant-based Characterization of the Aggregate Interference Power}

We introduced a simple yet comprehensive method to calculate the cumulants. Our method is applicable for finite and infinite networks, and is flexible to encompass different system and propagation parameters including large-scale fading, small-scale fading, and composite fading. We also highlighted the behavior of these cumulants with respect to changes in the network size and fading distributions. Moreover, we discussed some cumulants-based approximations of the distribution of the aggregate interference power.

\subsubsection{Gaussianity of the Distribution of the Aggregate Interference Power}

We studied the convergence of this distribution to a Gaussian distribution. Based on the Berry-Esseen bound, we casted in a single mathematical framework some observations scattered across the literature about the Gaussianity of the distribution of the aggregate interference power. We showed that an increase in the size of the exclusion region brings the distribution of the aggregate interference power closer to the Gaussian distribution. Increasing the active node density has a similar effect. However, the convergence is faster with increase in the size of the exclusion region compared to the increase in the active node density. In contrast, channel fading causes divergence from Gaussianity. Shadow fading typically causes more divergence as compared to multipath fading. 


\subsubsection{Impact of the Spatial Size of the Secondary Network on Spectrum Sharing}

One main contribution of this thesis is the study of the impact of the field size of secondary users on spectrum sharing opportunities. The study shows that an increase in the field size may eliminate the spectrum sharing opportunities. However, there are some cases where the spectrum sharing opportunities are not reduced by the increase in the field size even when the field size grows to infinity.

We demonstrated that asymptotic results obtained for an infinite field could be applied for a finite field whose radial depth is much greater than the minimum distance between the field and the primary user. Otherwise, these asymptotic results will be too conservative and may lead to missing spectrum sharing opportunities.

The results also show that under certain conditions it is possible for a secondary network to concurrently and continuously share the spectrum with a primary network without the need for spectrum sensing techniques or other cognitive radio functionalities. This observation hints a promising spectrum sharing solution for the wireless sensor networks which are facing interference challenges in the crowded unlicensed spectrum.

\subsubsection{Dominant Regions in Large Secondary Networks}

In this thesis, we investigated the dominant region that dictates the spectrum sharing opportunities of a large secondary network. We identified the boundary of this dominant region. We showed that as the exclusion region increases the dominant region increases as well. However, the dominant region shrinks with the increase in the path-loss exponent. The higher the required accuracy is, the wider the dominant region becomes. In addition, we demonstrated that the boundary of the dominant region shrinks with the increase of the interference threshold level. Results reported 
in this thesis are anticipated to be useful for designing MAC protocols and algorithms for large wireless secondary networks to create and maintain spectrum sharing opportunities.

\subsection{Suggested Future Work}

Following subsections provide some suggestions for possible extensions of the work presented in this thesis.

\subsubsection{Spatially Clustered Secondary Users}

In this thesis we focused on modeling the spatial distribution of active secondary networks by homogeneous PPP. While this modeling has many advantages, it might lead to conservative decisions hiding some spectrums sharing opportunities especially for clustered secondary users. An interesting extension of the work presented here is to model secondary users by cluster processes $[1,2,61,101]$, and to investigate the effect of this modeling on the conclusions deduced in this thesis.

\subsubsection{Exact Analysis of Spectrum Sharing for a Secondary Network Over- lapped with Spatially Distributed Many Primary Users}

The focus of this thesis was on most of the cases on scenarios including a single primary user. While we provided some generalization comments to account for scenarios having many primary users, a thorough investigation is advised for the cases when the secondary network is overlapped with a primary network whose (primary) users are spatially distributed according to a point process. A possible starting point for this investigation is to use Matern hard-core process $[2,10]$. 


\subsubsection{Further Accurate Approximating Distributions for the Aggregate Interference Power}

While we have used an approximating distribution that provides an acceptable accuracy in the region of interest, there are some techniques that could be explored to further enhance the accuracy of the approximation. One of these techniques is to approximate the distribution by a Gamma mixture. This technique allows the use of more moments (cumulants) in the approximation, which is anticipated to enhance the approximation accuracy. A starting point for exploring this technique is to apply the approach proposed in [80]. Another possible technique is to explore the possibility of approximating the distribution of the aggregate interference power by one of the heavy tailed distributions discussed in [98].

\subsubsection{Effect of Correlation among the $X_{i}$ 's}

An important assumption in our model is that $X_{i}$ 's are independent. However, there are some practical cases imposing the existence of correlation between $X_{i}$ 's. Therefore, it would be of an interest to investigate the effect of this correlation on the spectrum sharing, in particular, to investigate the effect of spatial correlation between shadow fading components in $X_{i}$ 's. A suggested approach to tackle this research problem is to use stochastic geometry techniques. In stochastic geometry, $X_{i}$ 's can be modeled as marks for the points that are distributed according to a point process, e.g., PPP. Some guidance could be taken from [102] that discusses correlation between marks in forestry statistics, which can be easily mapped to the problem in hand, i.e., correlated $X_{i}$ 's. 


\section{Bibliography}

[1] J. F. C. Kingman, Poisson Processes. New York: Oxford University Press, 1993.

[2] D. Stoyan, W. Kendall, and J. Mecke, Stochastic geometry and its applications, 2nd ed. John Wiley and Sons, 1996.

[3] M. Haenggi, J. G. Andrews, F. Baccelli, O. Dousse, and M. Franceschetti, "Stochastic geometry and random graphs for the analysis and design of wireless networks," IEEE J. Sel. Areas Commun., vol. 27, no. 7, pp. 1029-1046, Sep. 2009 .

[4] "Spectrum policy task force," Federal Communications Commission (FCC), ET Docket no. 02-135, Nov. 2002.

[5] S. Haykin, "Cognitive radio: brain-empowered wireless communications," IEEE J. Sel. Areas Commun., vol. 23, no. 2, pp. 201-220, Feb. 2005.

[6] I. Akyildiz, W. T. Lee, M. Vuran, and S. Mohanty, "Next generation/dynamic spectrum access/cognitive radio wireless networks: a survey," Comp. Networks $J$. , vol. 50, no. 13 , pp. 2127-2159, Sep. 2006.

[7] M. Aljuaid and H. Yanikomeroglu, "On the asymptotic analysis of average interference power generated by a wireless sensor network," in Proc. IEEE Vehicular Technology Conference (VTC) 2008-Fall, Calgary, AB, Canada, Sep. 2008. 
[8] H. S. Lichte, S. Valentin, and H. Karl, "Expected interference in wireless networks with geometric path loss: a closed-form approximation," IEEE Commun. Lett., vol. 14, no. 2, pp. 130-132, Feb. 2010.

[9] R. Menon, R. M. Buehrer, and J. H. Reed, "Outage probability based comparison of underlay and overlay spectrum sharing techniques," in Proc. 1st IEEE Symp. Dynamic Spectrum Access Networks (DySPAN'05), Baltimore, MD, USA, Nov. 2005, pp. 101-109.

[10] — "Impact of exclusion region and spreading in spectrum-sharing ad hoc networks," in Proc. First Internation Workshop on Technology and Policy for Accessing Spectrum, Boston, MA, USA, Aug. 2006.

[11] C. C. Chan and S. V. Hanly, "Calculating the outage probability in a CDMA network with spatial Poisson traffic," IEEE Trans. Veh. Technol., vol. 50, no. 1, pp. 183-204, Jan. 2001.

[12] A. Ghasemi and E. S. Sousa, "Interference aggregation in spectrum-sensing cognitive wireless networks," IEEE J. Sel. Topics Signal Process., vol. 2, no. 1, pp. 41-56, Feb. 2008.

[13] J. S. Evans and D. Everitt, "On the teletraffic capacity of CDMA cellular networks," IEEE Trans. Veh. Technol., vol. 48, no. 1, pp. 153-165, Jan. 1999.

[14] M. Z. Win, P. C. Pinto, and L. A. Shepp, "A mathematical theory of network interference and its applications," Proceedings of the IEEE, vol. 97, no. 2, pp. 205-230, Feb. 2009.

[15] A. Hasan and J. G. Andrews, "The guard zone in wireless ad hoc networks," IEEE Trans. Wireless Commun., vol. 6, no. 3, pp. 897-906, Mar. 2007. 
[16] R. K. Ganti and M. Haenggi, "Interference in ad hoc networks with general motion-invariant node distribution," in Proc. IEEE International Symposium on Information Theory (ISIT), Toronto, Canada, Jul. 2008.

[17] P. C. Pinto and M. Z. Win, "Spectral characterization of wireless networks," IEEE Wireless Commun. Mag., vol. 14, no. 6, pp. 27-31, Dec. 2007.

[18] "Spectrum commons classes for license-exemption," Office of Communications (OFCOM), Tech. Rep., Nov. 2008.

[19] R. Etkin, "Spectrum sharing: fundamental limits, scaling laws, and selfenforcing protocols," Ph.D. dissertation, EECS Department, University of California, Berkeley, CA, USA, 2006.

[20] S. Weber, J. Andrews, and N. Jindal, "The effect of fading, channel inversion, and threshold scheduling on ad hoc networks," IEEE Trans. Inf. Theory, vol. 53, no. 11, pp. 4127-4149, Nov. 2007.

[21] M. Aljuaid and H. Yanikomeroglu, "Impact of secondary users field size on spectrum sharing opportunities," in Proc. IEEE Wireless Communications and Networking Conference (WCNC), Sydney, Australia, Apr. 2010. [Online]. Available: http://www.sce.carleton.ca/faculty/yanikomeroglu/cv/publications.shtml

[22] _-, "A cumulant-based characterization of the aggregate interference power in wireless networks," in Proc. IEEE Vehicular Technology Conference (VTC) 2010-Spring, Taipei, Taiwan, May 2010.

[23] —-, "A cumulant-based investigation of the impact of secondary users' field size on spectrum sharing opportunities," submitted to IEEE Trans. Veh. Technol. (submission: 12 April 2010). 
[24] —_, "Investigating the validity of a Gaussian approximation for the distribution of the aggregate interference power in large wireless networks," in Proc. 25th Biennial Symposium on Communications (QBSC) 2010, Queen's University, Kingsotn, ON, Canada, May 2010.

[25] — - "Investigating the Gaussian convergence of the distribution of the aggregate interference power in large wireless networks," accepted with minor revisions in IEEE Trans. Veh. Technol. (20 April 2010).

[26] — - "Identifying boundaries of dominant regions dictating spectrum sharing opportunities for large secondary networks," in Proc. IEEE International Symposium on Personal, Indoor and Mobile Radio Communications (PIMRC) 2010, Istanbul, Turkey, Sep. 2010.

[27] "United States frequency allocations," National Telecommunications and Information Administration, USA, Chart, 2003. [Online]. Available: http://www.ntia.doc.gov/osmhome/allochrt.pdf

[28] I. Mitola, J. and J. Maguire, G.Q., "Cognitive radio: making software radios more personal," IEEE Personal Communications, vol. 6, no. 4, pp. 13 -18, Aug. 1999.

[29] J. Mitola, "Cognitive radio: an integrated agent architecture for software defined radio," Ph.D. dissertation, Royal Inst. Technol. (KTH), Stockholm, Sweden, 2000 .

[30] M. A. McHenry and K. Steadman, "Spectrum occupancy measurements, location 1 of 6: Riverbend Park, Great Falls, Virginia," Shared Spectrum Company, Report, Aug. 2005. 
[31] — , "Spectrum occupancy measurements, location 2 of 6: Tysons Square Center, Vienna, Virginia, April 9, 2004," Shared Spectrum Company, Report, Aug. 2005.

[32] M. A. McHenry and S. Chunduri, "Spectrum occupancy measurements, location 3 of 6: National Science Foundation Building roof, April 16, 2004, revision 2," Shared Spectrum Company, Report, Aug. 2005.

[33] M. A. McHenry, D. McCloskey, and G. Lane-Roberts, "Spectrum occupancy measurements, location 4 of 6: Republican National Convention, New York City, New York, August 30, 2004 - september 3, 2004, revision 2," Shared Spectrum Company, Report, Aug. 2005.

[34] M. A. McHenry and K. Steadman, "Spectrum occupancy measurements, location 5 of 6: National Radio Astronomy Observatory (NRAO), Green Bank, West Virginia, October 10 -11, 2004, revision 3," Shared Spectrum Company, Report, Aug. 2005.

[35] M. A. McHenry, D. McCloskey, and J. Bates, "Spectrum occupancy measurements, location 6 of 6: Shared Spectrum Building roof, Vienna, Virginia, December 15-16, 2004," Shared Spectrum Company, Report, Aug. 2005.

[36] J. Mitola, A. Attar, H. Zhang, O. Holland, H. Harada, and H. Aghvami, "Achievements and the road ahead: The first decade of cognitive radio," IEEE Trans. Veh. Technol., vol. 59, no. 4, pp. $1574-1577$, May 2010.

[37] "FCC adopted rules for unlicensed use of television white spaces," Federal Communications Commission (FCC), ET Docket no. 08-260A1, Nov. 2008.

[38] M. Marcus, "Wireless innovation and spectrum policy: the FCC opens a new inquiry," IEEE Wireless Communications, vol. 16, no. 5, pp. 4-5, Oct. 2009. 
[39] C. Stevenson, G. Chouinard, Z. Lei, W. Hu, S. Shellhammer, and W. Caldwell, "IEEE 802.22: The first cognitive radio wireless regional area network standard," IEEE Commun. Mag., vol. 47, no. 1, pp. 130 -138, Jan. 2009.

[40] M. Sherman, A. Mody, R. Martinez, C. Rodriguez, and R. Reddy, "IEEE standards supporting cognitive radio and networks, dynamic spectrum access, and coexistence," IEEE Communications Magazine, vol. 46, no. 7, pp. $72-79$, Jul. 2008 .

[41] "IEEE draft standard for information technology-telecommunications and information exchange between systems-local and metropolitan area networksspecific requirements-part 22.1: Standard to enhance harmful interference protection for low power licensed devices operating in the tv broadcast bands," IEEE Unapproved Draft Std P802.22.1/D6, Feb 2009, 2009.

[42] F. Granelli, P. Pawelczak, R. Prasad, K. Subbalakshmi, R. Chandramouli, J. Hoffmeyer, and H. Berger, "Standardization and research in cognitive and dynamic spectrum access networks: IEEE SCC41 efforts and other activities," IEEE Communications Magazine, vol. 48, no. 1, pp. 71 -79, Jan. 2010.

[43] "Standard ECMA-392 MAC and PHY for operation in TV white space," Dec. 2009. [Online]. Available: http://www.ecmainternational.org/publications/standards/Ecma-392.htm

[44] I. Akyildiz, W.-Y. Lee, M. Vuran, and S. Mohanty, "A survey on spectrum management in cognitive radio networks," IEEE Commun. Mag., vol. 46, no. 4, pp. $40-48$, Apr. 2008.

[45] Q. Zhao and B. Sadler, "A survey of dynamic spectrum access," IEEE Signal Process. Mag., vol. 24, no. 3, pp. 79 -89, May 2007. 
[46] A. Goldsmith, S. Jafar, I. Maric, and S. Srinivasa, "Breaking spectrum gridlock with cognitive radios: An information theoretic perspective," Proceedings of the IEEE, vol. 97, no. 5, pp. 894 -914, May 2009.

[47] P. Kolodzy, "Interference temperature: a metric for dynamic spectrum utilization," Int. J. Network Mgmt, vol. 16, no. 2, pp. 103-113, Mar. 2006.

[48] R. Menon, R. Buehrer, and J. Reed, "On the impact of dynamic spectrum sharing techniques on legacy radio systems," IEEE Transactions on Wireless Communications, vol. 7, no. 11, pp. $4198-4207$, Nov. 2008.

[49] M. Vu, N. Devroye, , and V. Tarokh, "On the primary exclusive region of cognitive networks," IEEE Trans. Wireless Commun., vol. 8, no. 7, pp. 33803385, Jul. 2009.

[50] M. Hanif, M. Shafi, P. Smith, and P. Dmochowski, "Interference and deployment issues for cognitive radio systems in shadowing environments," in IEEE International Conference on Communications (ICC '09), 14-18 2009, pp. 1 -6.

[51] Y. Zhao, D. Raymond, C. da Silva, J. H. Reed, and S. F. Midkiff, "Performance evaluation of radio environment map-enabled cognitive spectrum-sharing networks," in IEEE Military Communications Conference, MILCOM 2007., 29-31 2007, pp. $1-7$.

[52] S. Musa and W. Wasylkiwskyj, "Co-channel interference of spread spectrum systems in a multiple user environment," IEEE Trans. Commun., vol. 26, no. 10, pp. $1405-1413$, Oct. 1978.

[53] E. S. Sousa and J. A. Silvester, "Optimum transmission ranges in a directsequence spread-spectrum multihop packet radio network," IEEE J. Sel. Areas Commun., vol. 8, no. 5, pp. 762-771, Jun. 1990. 
[54] E. S. Sousa, "Performance of a spread spectrum packet radio network link in a Poisson field of interferers," IEEE Trans. Inf. Theory, vol. 38, no. 6, pp. 1743-1754, Nov. 1992.

[55] R. Mathar and J. Mattfeldt, "On the distribution of cumulated interference power in rayleigh fading channels," Wireless Networks, vol. 1, no. 1, pp. 31-36, Feb.-Mar. 1995.

[56] T. J. Shepard, "A channel access scheme for large dense packet radio networks," in Proc. of ACM SIGCOMM'96, Stanford, CA, USA, Aug. 1996. [Online]. Available: http://www.acm.org/sigcomm/sigcomm96/papers/shepard.ps

[57] M. Haenggi, "On distances in uniformly random networks," IEEE Trans. Inf. Theory, vol. 51, no. 10, pp. 3584-3586, Oct. 2005.

[58] S. Srinivasa and M. Haenggi, "Modeling interference in finite uniformly random networks," in Proc. International Workshop on Information Theory for Sensor Networks (WITS 2007), Santa Fe, USA, Jun. 2007.

[59] R. Hekmat and P. V. Mieghem, "Interference power statistics in ad-hoc and sensor networks," Wireless Networks, vol. 14, no. 5, pp. 591-599, Oct. 2008.

[60] "IEEE recommended practice for the analysis of in-band and adjacent band interference and coexistence between radio systems," IEEE Std P1900.2 - 2008, 2008.

[61] R. Ganti and M. Haenggi, "Interference and outage in clustered wireless ad hoc networks," IEEE Transactions on Information Theory, vol. 55, no. 9, pp. 4067 -4086 , Sep. 2009. 
[62] E. Salbaroli and A. Zanella, "Interference analysis in a Poisson field of nodes of finite area," IEEE Trans. Veh. Technol., vol. 58, no. 4, pp. 1776-1783, May 2009 .

[63] Y. M. Shobowale and K. A. Hamdi, "A unified model for interference analysis in unlicensed frequency bands," IEEE Trans. Wireless Commun., vol. 8, no. 8, pp. 4004-4013, Aug. 2009.

[64] T. Brown, "A harmful interference model for unlicensed device operation in licensed service bands," Journal of Communications, vol. 1, no. 1, 2006. [Online]. Available: http://ojs.academypublisher.com/index.php/jcm/article/view/90

[65] J. Ilow and D. Hatzinakos, "Analytic alpha-stable noise modeling in a Poisson field of interferers or scatterers," IEEE Trans. on Signal Process., vol. 46, no. 6, pp. $1601-1611$, Jun. 1998.

[66] X. Yang and A. P. Petropulu, "Co-channel interference modeling and analysis in a Poisson field of interferers in wireless communications," IEEE Trans. Signal Process., vol. 51, no. 1, pp. 64-76, Jan. 2003.

[67] F. Baccelli, B. Blaszczyszyn, and P. Muhlethaler, "An Aloha protocol for multihop mobile wireless networks," IEEE Trans. Inf. Theory, vol. 52, no. 2, pp. $421-436$, Feb. 2006.

[68] M. Shao and C. Nikias, "Signal processing with fractional lower order moments: stable processes and their applications," Proceedings of the IEEE, vol. 81, no. 7, pp. $986-1010$, Jul. 1993.

[69] A. A. Abu-Dayya and N. C. Beaulieu, "Outage probabilities in the presence of correlated lognormal interferers," IEEE Transactions on Vehicular Technology, vol. 43, no. 1, pp. $164-173$, Feb. 1994. 
[70] N. C. Beaulieu, A. A. Abu-Dayya, and P. J. McLane, "Estimating the distribution of a sum of independent lognormal random variables," IEEE Transactions on Communications, vol. 43, no. 12, pp. 2869-2873, Dec. 1995.

[71] F. Berggren and S. B. Slimane, "A simple bound on the outage probability with lognormally distributed interferers," IEEE Commun. Lett., vol. 8, no. 5, pp. 271-273, May 2004.

[72] N. C. Beaulieu and Q. Xie, "An optimal lognormal approximation to lognormal sum distributions," IEEE Transactions on Vehicular Technology, vol. 53, no. 2, pp. 479 - 489, Mar. 2004.

[73] N. C. Beaulieu and F. Rajwani, "Highly accurate simple closed-form approximations to lognormal sum distributions and densities," IEEE Communications Letters, vol. 8, no. 12, pp. 709 - 711, Dec. 2004.

[74] J. Hu and N. C. Beaulieu, "Accurate simple closed-form approximations to Rayleigh sum distributions and densities," IEEE Communications Letters, vol. 9, no. 2, pp. 109 - 111, Feb. 2005.

[75] _ _ "Accurate closed-form approximations to Ricean sum distributions and densities," IEEE Communications Letters, vol. 9, no. 2, pp. 133 - 135, Feb. 2005 .

[76] N. Mehta, J. Wu, A. Molisch, and J. Zhang, "Approximating a sum of random variables with a lognormal," IEEE Transactions on Wireless Communications, vol. 6 , no. 7 , pp. $2690-2699$, Jul. 2007.

[77] A. Hyvarinen, J. Karhunen, and E. Oja, Independent Component Analysis. New York, USA: Wiley, 2001. 
[78] A. Stuart and J. K. Ord, Kendall's Advanced Theory of Statistics, 6th ed. London, UK: Edward Arnold, 1994.

[79] M. Abramowitz and I. A. Stegun, Handbook of Mathematical Functions with Formulas, Graphs, and Mathematical Tables, 10th ed. Washington, D.C.: U.S. Government Printing Office, 1972.

[80] B. G. Lindsay, R. S. Pilla, and P. Basak, "Moment-based approximations of distributions using mixtures: theory and applications," Annals of the Institute of Statistical Mathematics, vol. 52, no. 2, pp. 215-230, June 2000.

[81] A. Iyer, C. Rosenberg, and A. Karnik, "What is the right model for wireless channel interference?" IEEE Transactions on Wireless Communications, vol. 8, no. 5, pp. $2662-2671$, may 2009.

[82] A. F. Molisch, L. J. Greenstein, and M. Shafi, "Propagation issues for cognitive radio," Proceedings of the IEEE, vol. 97, no. 5, pp. 787-804, May 2009.

[83] H. Inaltekin, M. Chiang, H. V. Poor, and S. B. Wicker, "On unbounded pathloss models: effects of singularity on wireless network perfomance," IEEE $J$. Sel. Areas Commun., vol. 27, no. 7, pp. 1078-1091, Sep. 2009.

[84] J. W. Gluck and E. Geraniotis, "Throughput and packet error probability in cellular frequency-hopped spread spectrum radio networks," IEEE J. Sel. Areas Commun., vol. 7, no. 1, pp. 148-160, Jan. 1989.

[85] G. Zhou, J. A. Stankovic, and S. H. Son, "Crowded spectrum in wireless sensor networks," in Proc. of EmNets 2006, Cambridge, MA, USA, May 2006.

[86] "Wireless sensor networks: a survey," Computer Networks, vol. 38, no. 4, pp. $393-422,2002$. 
[87] "IEEE standard for information technology- telecommunications and information exchange between systems- local and metropolitan area networks- specific requirements part 15.4: Wireless medium access control (MAC) and physical layer (PHY) specifications for low-rate wireless personal area networks (WPANs)," IEEE Std 802.15.4-2006 (Revision of IEEE Std 802.15.4-2003), 2006.

[88] F. Granelli, H. Zhang, X. Zhou, and S. Maranò, "Research advances in cognitive ultra wide band radio and their application to sensor networks," Mobile Networks and Applications, vol. 11, no. 4, pp. 487-499, Aug. 2006.

[89] E. W. Weisstein, "Generalized hypergeometric function," from MathWorld-A Wolfram Web Resource. [Online]. Available: http://mathworld.wolfram.com/GeneralizedHypergeometricFunction.html

[90] A. Leon-Garcia, Probability and Random Processes for Electrical Engineering, 2nd ed. Reading, Massachusetts: Addison-Wesley, 1994.

[91] H. Chernoff, "A measure of asymptotic efficiency for tests of a hypothesis based on the sum of observations," The Annals of Mathematical Statistics, vol. 23, no. 4, pp. 493 - 507, Dec. 1952. [Online]. Available: http://www.jstor.org/stable/2236576

[92] W. Feller, An Introduction to Probability Theory and Its Applications, 2nd ed. New York: Wiley, 1971.

[93] P. M. Shankar, "Perfromance analysis of diversity combining algorithms in shadowed fading channels," Wireless Personal Communications, vol. 37, no. 1-2, pp. 61-72, Apr. 2006. 
[94] D. E. Barton and K. E. Dennis, "The conditions under which Gram-Charlier and Edgeworth curves are positive definite and unimodal," Biometrika, vol. 39, 1952.

[95] K. L. Q. Read, "A lognormal approximation for the collector's problem," The American Statistician, vol. 52, no. 2, pp. 175-180, May 1998.

[96] L. L. Cam, "The central limit theorem around 1935," Statistical Science, vol. 1, no. 1 , pp. $78-91,1986$.

[97] J. Fiorina and W. Hachem, "On the asymptotic distribution of the correlation receiver output for time-hopped UWB signals," IEEE Trans. Signal Process., vol. 54, no. 7, pp. 2529-2545, Jul. 2006.

[98] N. C. Beaulieu and D. J. Young, "Designing time-hopping ultrawide bandwidth receivers for multiuser interference environments," Proceedings of the IEEE, vol. 97 , no. 2 , pp. $255-284$, Feb. 2009.

[99] L. Heinrich and V. Schmidt, "Normal convergence of multidimensional shot noise and rates of this convergence," Adv. Appl. Prob., vol. 17, pp. 709-730, 1985.

[100] J. A. Lane, "The Berry-Essen bound for the Poisson shot-noise," Adv. Appl. Prob., vol. 19, pp. 512-514, 1987.

[101] K. Gulati, B. L. Evans, J. G. Andrews, and K. R. Tinsley, "Statistics of cochannel interference in a field of poisson and poisson-poisson clustered interferers," submitted to IEEE Trans. Signal Process. (submission: November 2009).

[102] D. Stoyan and A. Penttinen, "Recent applications of point process methods in forestry statistics," Statistical Science, vol. 15, no. 1, pp. 61-78, Feb. 2000. 


\section{Appendices}




\section{Appendix A}

\section{The Berry-Esseen Theorem}

The following theorem describes the Berry-Esseen bound in its basic form.

Theorem A.0.1 (Berry-Esseen Theorem [92]).

Let $\left\{Y_{j}\right\}$ be independent random variables with a common cumulative distribution

function (CDF) $F$, with zero mean, non-zero variance $\left(\sigma^{2}>0\right)$, and finite third absolute moment $\left(\rho=\mathbb{E}\left[\left|Y_{j}\right|^{3}\right]<\infty\right)$. Then for all $y$ and $k$

$$
\left|F_{k}(y)-F_{N}(y)\right| \leq \frac{3 \rho}{\sigma^{3} \sqrt{k}}
$$

where $F_{k}$ is the CDF of the normalized sum $Z=\frac{1}{\sigma \sqrt{k}} \sum_{j=1}^{k} Y_{j}$, and $F_{N}$ is the CDF of the standard normal distribution, i.e., $\mathcal{N}(0,1)$.

Proof.

The proof can be found in [92, p. 543].

According to [92], the factor 3 on the right is not claimed to be the optimal value. Therefore, it is expected that different references may propose smaller values. 


\section{Appendix B}

\section{Derivation of the Cumulant-Based Berry-Esseen}

\section{Bound}

The shot noise considered in [99] is assumed to be generated by a stationary marked point process defined in $\mathbb{R}^{d} \times \mathbb{K}$ where $\mathbb{R}^{d}$ is a $d$-dimensional Euclidian space and $\mathbb{K}$ is a space of marks. This shot noise has the form

$$
\Xi(t)=\sum_{i} f\left(t-q_{i}, x_{i}\right)
$$

where $f$ is a real-valued function denoting the effect at $t \in \mathbb{R}^{d}$ caused by an event at a random position $q_{i} \in \mathbb{R}^{d}$ with an independent random mark $x_{i} \in \mathbb{K}$. The marks $x_{i}$ are assumed to be mutually independent. According to Theorem 7 in [99], the Berry-Esseen bound for the normalized shot noise $Z$ when the underlying process is a stationary PPP can be written as

$$
\left|F_{Z}(y)-F_{N}(y)\right| \leq 2.21 \frac{H_{3}}{\sqrt{\lambda}\left(H_{2}\right)^{3 / 2}}
$$

where $H_{3}=\int_{\mathbb{R}^{d}} \mathbb{E}_{X}\left[\left|f\left(u, x_{i}\right)\right|^{3}\right] d u$ and $H_{2}=\int_{\mathbb{R}^{d}} \mathbb{E}_{X}\left[f^{2}\left(u, x_{i}\right)\right] d u$. Within the context of our thesis, the function $f$ represents an individual interference power which is positive. Therefore, $H_{3}$ can be written without the absolute value function as $H_{3}=$ 
$\int_{\mathbb{R}^{d}} \mathbb{E}_{X}\left[f^{3}\left(u, x_{i}\right)\right] d u$.

To relate $H_{3}$ and $H_{2}$ to the cumulants of the shot noise in (B.1), the characteristic function of $\Xi$ should be determined first. Based on Campbell's theorem [1], the characteristic function of the sum in (B.1), which is over a stationary and independently marked PPP, can be written as

$$
\phi(\omega)=\exp \left(\lambda \int_{\mathbb{R}^{d}} \int_{\mathbb{K}}(\exp (j \omega f(u, x))-1) f_{X}(x) d x d u\right),
$$

where $f_{X}$ is the PDF of $X$. From (B.3), the $m$ th cumulant can be obtained by

$$
\begin{aligned}
\kappa_{m} & =\frac{1}{j^{m}}\left[\frac{d^{m} \ln (\phi(\omega))}{d \omega^{m}}\right]_{\omega=0} \\
& =\lambda \int_{\mathbb{R}^{d}} \mathbb{E}_{X}\left[f^{m}(u, x)\right] d u .
\end{aligned}
$$

Comparing (B.4) to the expressions for $H_{3}$ and $H_{2}$ yields $\kappa_{2}=\lambda H_{2}$ and $\kappa_{3}=$ $\lambda H_{3}$. Thus, the Berry-Esseen bound can be written in terms of the second and third cumulants as

$$
\left|F_{Z}(y)-F_{N}(y)\right| \leq 2.21 \frac{\kappa_{3}}{\kappa_{2}^{3 / 2}} .
$$

It is worth noting that $\kappa_{3} /\left(\kappa_{2}\right)^{3 / 2}$ reflects the skewness of $\Xi$. 


\section{Appendix $\mathrm{C}$}

\section{Generalization of the Berry-Esseen Bound for a $d$-dimensional Space}

Results reported in [99] and [100] for the Berry-Esseen bound are applicable for a multidimensional space. Therefore, we can use (6.1) to investigate the Gaussianity of the aggregate interference power of a wireless network deployed in a $d$-dimensional space. However, we need to evaluate cumulants of a $d$-dimensional space. To achieve this, we utilize cumulants expressions provided in [58] and we extend them to our problem. As a result, we have the following expression for the $m$ th cumulant in a d-dimensional space:

$$
\kappa_{m}\left(I_{A}\right)=\frac{d b_{d} \lambda}{m n-d} r_{o}^{d-m n} \tilde{\mu}_{m}(X)
$$

where $b_{d}$ is the volume of the unit ball in $\mathbb{R}^{d}$, which is equal to $\frac{\pi^{\frac{d}{2}}}{\Gamma\left(1+\frac{d}{2}\right)}$, and $\Gamma($.$) is the$ gamma function. To avoid divergence of the interference, these results are applicable for $n>d$. Using (6.1) and (C.1), the Berry-Esseen bound becomes

$$
\left|F_{Z}(y)-F_{N}(y)\right| \leq 2.21 \frac{(2 n-d)^{\frac{3}{2}}}{d^{\frac{1}{2}}(3 n-d)} \frac{1}{\sqrt{\lambda v_{d}\left(0, r_{o}\right)}} \frac{\tilde{\mu}_{3}(X)}{\left[\tilde{\mu}_{2}(X)\right]^{\frac{3}{2}}}
$$

These expressions are applicable when $r_{o} \geq r_{c}$. Expressions for the other case, i.e., $r_{o}<r_{c}$, can be also obtained in a similar way. 